\title{
Gulkana Glacier, Alaska-Mass Balance, Meteorology, and Water Measurements, 1997-2001
}

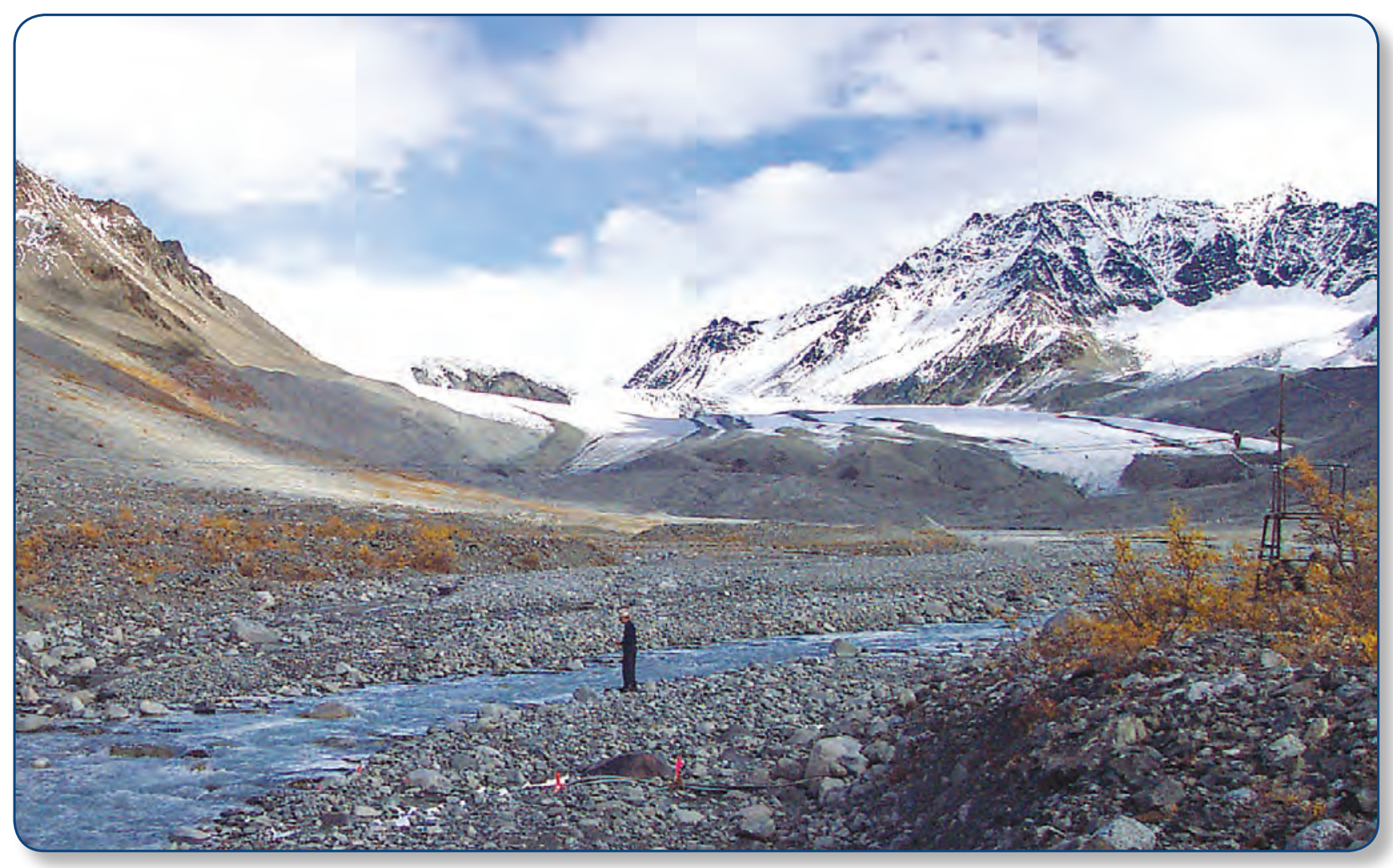

Scientific Investigations Report 2011-5046

U.S. Department of the Interior

U.S. Geological Survey 
Cover: Photograph looking at the mostly debris-covered Gulkana Glacier terminus from just downstream of Phelan Creek USGS streamflow-gaging station No. 15478040, September 12 , 2001. The station site is about 1 kilometer downstream of the present glacier terminus. Dennis Trabant, USGS retired, is standing at the edge of the creek in the center of the photograph. The channel bed is composed of typical ground moraine material-poorly sorted gravel and small boulders - which is subject to frequent changes during high flows. The mean discharge for September 12, 2001, was about 0.8 cubic meters per second (daily runoff of about 5.5 millimeters), a value about 40 percent of normal for this time time of year. The unmanned cableway (faintly visible) and southeast bank tower shown are used for making discharge measurements when the flow is too deep and fast for wading. The instrumentation and satellite telemetry shelter for the gaging station lie just beyond of the right edge of the photograph. A recent new fall snowfall is apparent at higher elevations. Photograph by Rod March. 


\section{Gulkana Glacier, Alaska-Mass Balance, Meteorology, and Water Measurements, 1997-2001}

By Rod S. March and Shad O'Neel

Scientific Investigations Report 2011-5046 


\title{
U.S. Department of the Interior \\ KEN SALAZAR, Secretary \\ U.S. Geological Survey \\ Marcia K. McNutt, Director
}

\author{
U.S. Geological Survey, Reston, Virginia: 2011
}

For more information on the USGS - the Federal source for science about the Earth, its natural and living resources, natural hazards, and the environment, visit http://www.usgs.gov or call 1-888-ASK-USGS.

For an overview of USGS information products, including maps, imagery, and publications, visit http://www.usgs.gov/pubprod

To order this and other USGS information products, visit http://store.usgs.gov

Any use of trade, product, or firm names is for descriptive purposes only and does not imply endorsement by the U.S. Government.

Although this report is in the public domain, permission must be secured from the individual copyright owners to reproduce any copyrighted materials contained within this report.

Suggested citation:

March, R.S., and O'Neel, Shad, 2011, Gulkana Glacier, Alaska-Mass balance, meteorology, and water measurements, 1997-2001: U.S. Geological Survey Scientific Investigations Report 2011-5046, 72 p. 


\section{Contents}

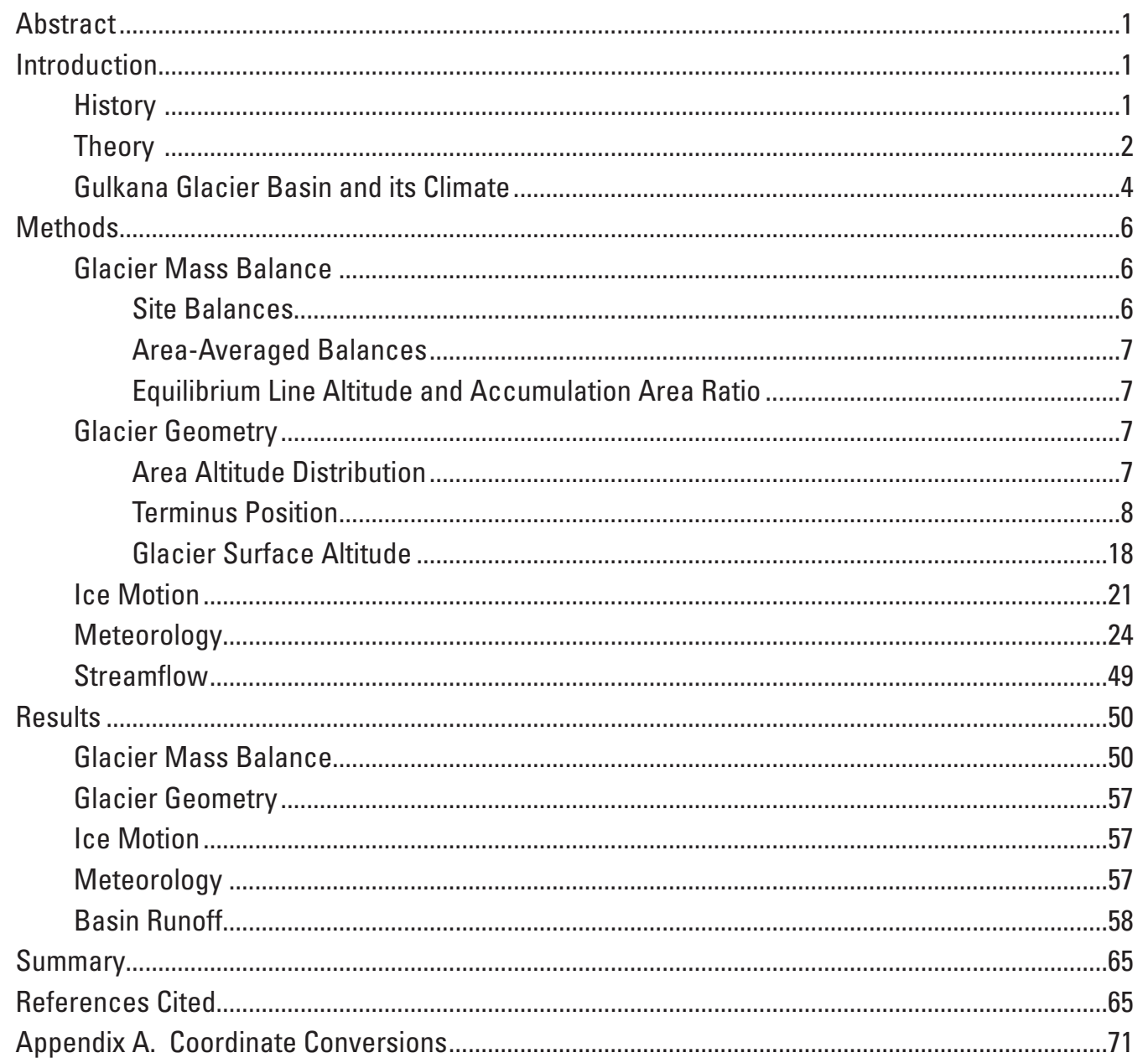




\section{Figures}

Figure 1. Diagram showing cause and effect relationships for data presented in this report

Figure 2. Map showing index measurement sites A, B, and D, glacier surface altitudes, weather and stream-gaging stations, and boundary of Gulkana Glacier, Alaska

Figure 3. Composite vertical photograph showing index measurement sites, stream-gaging station, weather station, and basin boundary, Gulkana Glacier, Alaska, July 11, 1993

Figure 4. Graph showing area altitude distribution of Gulkana Glacier, Alaska, in 1900, 1967, 1974, 1993, and 1999 for 100-meter altitude bins

Figure 5. Graph showing glacier terminus positions from Global Positioning System surveys at Gulkana Glacier, Alaska, in 1996 10

Figure 6. Graphs showing surface altitudes at index sites A, B, and D, Gulkana Glacier, Alaska 19

Figure 7. Graph showing locations of stakes at site B, Gulkana Glacier, Alaska, shown as an example

Figure 8. Graphs showing daily values of mean air temperature, precipitation catch, and daily runoff at Phelan Creek, Alaska, water year 1997

Figure 9. Graphs showing daily values of mean air temperature, precipitation catch, and daily runoff at Phelan Creek, Alaska, water year 1998

Figure 10. Graphs showing daily values of mean air temperature, precipitation catch, and daily runoff at Phelan Creek, Alaska, water year 1999

Figure 11. Graphs showing daily values of mean air temperature, precipitation catch, and daily runoff at Phelan Creek, Alaska, water year 2000.

Figure 12. Graphs showing daily values of mean air temperature, precipitation catch, and daily runoff at Phelan Creek, Alaska, water year 2001

Figure 13. Graphs showing difference between analog and digital temperature sensors at 1,480 meters altitude, Gulkana Glacier, Alaska, water years 1996-98

Figure 14. Graph showing monthly average wind speed at 1,480 meters altitude, Gulkana Glacier basin, Alaska, water years 1997-2001....

Figure 15. Photograph looking towards the Gulkana Glacier terminus from just downstream of the Phelan Creek stream-gaging station, USGS station 15478040, Alaska, September 1, 1996.

Figure 16. Graphs showing net, winter, and summer values for mass balance, air temperature, precipitation catch, and runoff, Gulkana Glacier basin, Alaska, water years 1966-2001

Figure 17. Graph showing cumulative conventional net balance and the cumulative reference surface net balance using the reference surface area altitude distribution of 1967, Gulkana Glacier, Alaska

Figure 18. Graphs showing precipitation gage catch efficiency relative to wind speed of the U.S. Geological Survey gage shield type in the Gulkana Glacier basin, Alaska, compared to the double fenced intercomparison reference shield for precipitation events greater than 3 millimeters during the winter 1997-98 at Toronto, Ontario 


\section{Tables}

Table 1. Coordinates of index sites A, B, C, and D ............................... 6

Table 2. Gulkana Glacier area altitude distribution, from Little Ice Age (LIA)

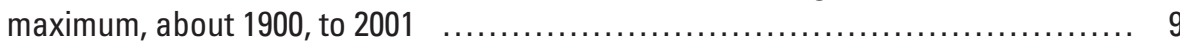

Table 3. Optical surveys of terminus position, Gulkana Glacier, March 25, 1985 .......... 10

Table 4. Photogrametric surveys of terminus positions, Gulkana Glacier, July 11, 1993

Table 5. Global Positioning System terminus position surveys, Gulkana Glacier, September 3, 1997

Table 6. Global Positioning System terminus position surveys, Gulkana Glacier, September 9, 1998

Table 7. Global Positioning System terminus-position surveys, Gulkana Glacier, September 16, 1999

Table 8. Global Positioning System terminus-position surveys, Gulkana Glacier, October 2, 2000

Table 9. Global Positioning System terminus-position surveys, Gulkana Glacier, August 23, 2001

Table 10. Gulkana Glacier glacier-surface altitudes at index sites A, B, and D ........... 20

Table 11. Glacier surface slope, stake bottom locations, and stake motion determined from optical surveys, Site A, 1996-2001

Table 12. Glacier surface slope, stake bottom locations, and stake motion determined from optical surveys, Site B, 1996-2001

Table 13. Glacier surface slope, stake bottom locations, and stake motion determined

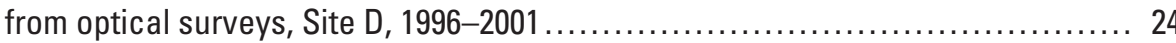

Table 14. Daily, monthly, and annual average air temperature from analog sensor at 1,480 meters altitude, Gulkana Glacier basin, 1997 hydrologic year .............. 30

Table 15. Daily, monthly, and annual average air temperature from analog sensor at 1,480 meters altitude, Gulkana Glacier basin, 1998 hydrologic year ............... 32

Table 16. Daily, monthly, and annual average air temperature from digital sensor at 1,480 meters altitude, Gulkana Glacier basin, 1999 hydrologic year ....

Table 17. Daily, monthly, and annual average air temperature from digital sensor at 1,480 meters altitude, Gulkana Glacier basin, 2000 hydrologic year ....

Table 18. Daily, monthly, and annual average air temperature from digital sensor at 1,480 meters altitude, Gulkana Glacier basin, 2001 hydrologic year .............. 36

Table 19. Daily, monthly, and annual precipitation catch recorded at 1,480 meters altitude, Gulkana Glacier basin, 1997 hydrologic year ...................... 38

Table 20. Daily, monthly, and annual precipitation catch recorded at 1,480 meters altitude, Gulkana Glacier basin, 1998 hydrologic year

Table 21. Daily, monthly, and annual precipitation catch recorded at 1,480 meters altitude, Gulkana Glacier basin, 1999 hydrologic year

Table 22. Daily, monthly, and annual precipitation catch recorded at 1,480 meters altitude, Gulkana Glacier basin, 2000 hydrologic year

Table 23. Daily, monthly, and annual precipitation catch recorded at 1,480 meters altitude, Gulkana Glacier basin, 2001 hydrologic year

Table 24. Daily, monthly, and annual average wind speed at 1,480 meters altitude, Gulkana Glacier basin, 1997 hydrologic year. 


\section{Tables-Continued}

Table 25. Daily, monthly, and annual average wind speed at 1,480 meters altitude,

Gulkana Glacier basin, 1998 hydrologic year.....

Table 26. Daily, monthly, and annual average wind speed at 1,480 meters altitude, Gulkana Glacier basin, 1999 hydrologic year....

Table 27. Daily, monthly, and annual average wind speed at 1,480 meters altitude, Gulkana Glacier basin, 2000 hydrologic year....

Table 28. Daily, monthly, and annual average wind speed at 1,480 meters altitude, Gulkana Glacier basin, 2001 hydrologic year ............................... 48

Table 29. Index-site and area-integrated conventional balance quantities for Gulkana Glacier and Gulkana Glacier basin, 1997-2001 hydrologic years

Table 30. Index-site and area-integrated reference surface balance quantities for Gulkana Glacier and Gulkana Glacier Basin, 1997-2001 hydrologic years

Table 31. Conventional and reference surface yearly and cumulative net balances and cumulative volume changes

Table 32. Daily mean, monthly, and annual runoff from the Gulkana Glacier Basin, 1997 hydrologic year

Table 33. Daily mean, monthly, and annual runoff from the Gulkana Glacier Basin, 1998 hydrologic year

Table 34. Daily mean, monthly, and annual runoff from the Gulkana Glacier Basin, 1999 hydrologic year

Table 35. Daily mean, monthly, and annual runoff from the Gulkana Glacier Basin, 2000 hydrologic year

Table 36. Daily mean, monthly, and annual runoff from the Gulkana Glacier Basin, 2001 hydrologic year 


\title{
Conversion Factors, Datums, and Abbreviations and Acronyms
}

\author{
Conversion Factors
}

\begin{tabular}{|c|c|c|}
\hline Multiply & By & To obtain \\
\hline \multicolumn{3}{|c|}{ Length } \\
\hline centimeter (cm) & 0.3937 & inch (in.) \\
\hline millimeter (mm) & 0.03937 & inch (in.) \\
\hline meter $(\mathrm{m})$ & 3.281 & foot $(\mathrm{ft})$ \\
\hline kilometer (km) & 0.6214 & mile (mi) \\
\hline kilometer (km) & 0.5400 & mile, nautical (nmi) \\
\hline meter $(\mathrm{m})$ & 1.094 & yard (yd) \\
\hline \multicolumn{3}{|c|}{ Area } \\
\hline square kilometer $\left(\mathrm{km}^{2}\right)$ & 247.1 & acre \\
\hline square kilometer $\left(\mathrm{km}^{2}\right)$ & 0.3861 & square mile $\left(\mathrm{mi}^{2}\right)$ \\
\hline \multicolumn{3}{|c|}{ Volume } \\
\hline cubic meter $\left(\mathrm{m}^{3}\right)$ & 6.290 & barrel (petroleum, 1 barrel = 42 gal) \\
\hline cubic meter $\left(\mathrm{m}^{3}\right)$ & 264.2 & gallon (gal) \\
\hline cubic meter $\left(\mathrm{m}^{3}\right)$ & 0.0002642 & million gallons (Mgal) \\
\hline cubic meter $\left(\mathrm{m}^{3}\right)$ & 35.31 & cubic foot $\left(\mathrm{ft}^{3}\right)$ \\
\hline cubic meter $\left(\mathrm{m}^{3}\right)$ & 1.308 & cubic yard $\left(\mathrm{yd}^{3}\right)$ \\
\hline cubic meter $\left(\mathrm{m}^{3}\right)$ & 0.0008107 & acre-foot (acre-ft) \\
\hline cubic kilometer $\left(\mathrm{km}^{3}\right)$ & 0.2399 & cubic mile $\left(\mathrm{mi}^{3}\right)$ \\
\hline liter (L) & 33.81 & ounce, fluid (fl. oz) \\
\hline liter (L) & 2.113 & pint (pt) \\
\hline liter (L) & 1.057 & quart (qt) \\
\hline liter (L) & 0.2642 & gallon (gal) \\
\hline \multicolumn{3}{|c|}{ Flow rate } \\
\hline cubic meter per second $\left(\mathrm{m}^{3} / \mathrm{s}\right)$ & 70.05 & acre-foot per day (acre-ft/d) \\
\hline cubic meter per second $\left(\mathrm{m}^{3} / \mathrm{s}\right)$ & 35.31 & cubic foot per second $\left(\mathrm{ft}^{3} / \mathrm{s}\right)$ \\
\hline meter per second (m/s) & 3.281 & foot per second (ft/s) \\
\hline meter per year (m/yr) & 3.281 & foot per year (ft/yr) \\
\hline millimeter per day (mm/d) & 0.03937 & inch per day (in/d) \\
\hline \multicolumn{3}{|c|}{ Mass } \\
\hline kilogram (kg) & 2.205 & pound avoirdupois (lb) \\
\hline \multicolumn{3}{|c|}{ Density } \\
\hline kilogram per liter (kg/L) & 62.43 & $\begin{array}{l}\text { pound avoirdupois per per cubic } \\
\text { foot }\left(\mathrm{lb} / \mathrm{ft}^{3}\right)\end{array}$ \\
\hline \multicolumn{3}{|c|}{ Plane angle } \\
\hline grad & 0.9 & degree \\
\hline
\end{tabular}

Temperature in degrees Celsius $\left({ }^{\circ} \mathrm{C}\right)$ may be converted to degrees Fahrenheit $\left({ }^{\circ} \mathrm{F}\right)$ as follows:

${ }^{\circ} \mathrm{F}=\left(1.8 x^{\circ} \mathrm{C}\right)+32$. 


\section{Conversion Factors, Datums, and Abbreviations and Acronyms-Continued}

The water year, or hydrologic year, is the interval between 0ctober 1 and the end of the following September and provides a framework for a fixed-date or annual mass balance estimate. Each estimate is designated by the calendar year in which it ends; for example, B98 represents the glacier-wide balance for the interval October 1, 1997-September 30, 1998). The terms "this year" and "current year" are used interchangeably in this report for balance year and water year, depending on the data being discussed.

All balance values are reported in meters weq. The density of water is $1 \mathrm{~kg} / \mathrm{L}$, and that of glacier ice is assumed to be $0.9 \mathrm{~kg} / \mathrm{L}$. We report density in kilograms per liter, which numerically is equivalent to the unitless relative density (the decimal fraction of the density of water).

Positions are given in a local horizontal reference frame (values $0-10,000 \mathrm{~m}$ ), Universal Transverse Mercator (UTM), and geographic coordinates (latitude, longitude). Horizontal coordinates in the local system (a sea-level-scale network based on UTM with the positive Y-axis approximately true north) may be converted to UTM zone 6 coordinates (NAD 83 datum) as described in appendix 1. NAD 83 datum coordinates are approximately equal to WGS 84 datum coordinates.

Vertical coordinates are referenced to the NGVD 29 datum. Within the basin and nearby region, these may be converted to the NAVD 88 datum or NAD 83 ellipsoid heights (approximately the same as WGS 84 ellipsoid heights) as described in appendix 1.

Abbreviations and Acronyms

AAD area-altitude distribution

AAR accumulation-area ratio

DD degree day

DRG digital raster graphic

ELA equilibrium line altitude

LIA Little Ice Age

mweq meters water equivalent

PPS Precise Positioning Service

USGS U.S. Geological Survey

UTM Universal Transverse Mercator

WGMS World Glacier Monitoring Service 


\title{
Gulkana Glacier, Alaska-Mass Balance, Meteorology, and Water Measurements, 1997-2001
}

\author{
By Rod S. March and Shad O'Neel
}

\begin{abstract}
The measured winter snow, maximum winter snow, net, and annual balances for 1997-2001 in the Gulkana Glacier basin are determined at specific points and over the entire glacier area using the meteorological, hydrological, and glaciological data. We provide descriptions of glacier geometry to aid in estimation of conventional and reference surface mass balances and descriptions of ice motion to aid in the understanding of the glacier's response to its changing geometry. These data provide annual estimates for area altitude distribution, equilibrium line altitude, and accumulation area ratio during the study interval. New determinations of historical area altitude distributions are given for 1900 and annually from 1966 to 2001. As original weather instrumentation is nearing the end of its deployment lifespan, we provide new estimates of overlap comparisons and precipitation catch efficiency.

During 1997-2001, Gulkana Glacier showed a continued and accelerated negative mass balance trend, especially below the equilibrium line altitude where thinning was pronounced. Ice motion also slowed, which combined with the negative mass balance, resulted in glacier retreat under a warming climate. Average annual runoff augmentation by glacier shrinkage for 1997-2001 was 25 percent compared to the previous average of 13 percent, in accordance with the measured glacier volume reductions.
\end{abstract}

\section{Introduction}

The U.S. Geological Survey (USGS) operates a long-term program to monitor glacier mass balance, geometry, motion and runoff while simultaneously monitoring local climate. Research aims include improved quantitative estimates of water resources, glacier-related hazards, and glacier response to global climate change (Fountain and others, 1997). These goals require making glaciological point measurements alongside continuous observations of local meteorology and runoff thereby facilitating glacier-wide balance estimates.

The purpose of this study is to establish long-term mass balance monitoring programs at three widely spaced index glaciers in the United States that sample different climateglacier-runoff regimes. This report focuses on Gulkana Glacier, one of the index glaciers located in interior Alaska. Other reports document the programs at Wolverine Glacier in south-central Alaska (e.g. Mayo and others, 2004) and South Cascade Glacier in Washington (e.g. Bidlake and others, 2007). This report contains the glaciological mass balance, geometry, ice dynamics, meteorological, and water budget measurements made at Gulkana Glacier for the 1997-2001 balance years (fig. 1).

\section{History}

Measurements began on Gulkana Glacier during the early 1960s with the University of Alaska Gulkana Glacier Project (Péwé and Reger, 1983). For several years this project measured the energy budget, mass balance, meteorology, foliation, flow, and glacier-bottom topography at Gulkana Glacier. In 1966, USGS initiated a suite of continuous measurements as part of the United States contribution to the International Hydrologic Decade study of glacier mass balance. After a decade of dense spatial sampling, the number of glacier measurement sites was reduced to three representative index sites, at which ice-motion and surfacealtitude observations (for determining glacier-volume change) began. The Gulkana Glacier record (36 years of balance data, 35 years of meteorology) now exceeds the 30 -year criterion to provide statistics with sufficient confidence that can be used in the selection of stations for international exchange through the Global Telecommunications Service for global climate monitoring (Karl and others, 1989). 


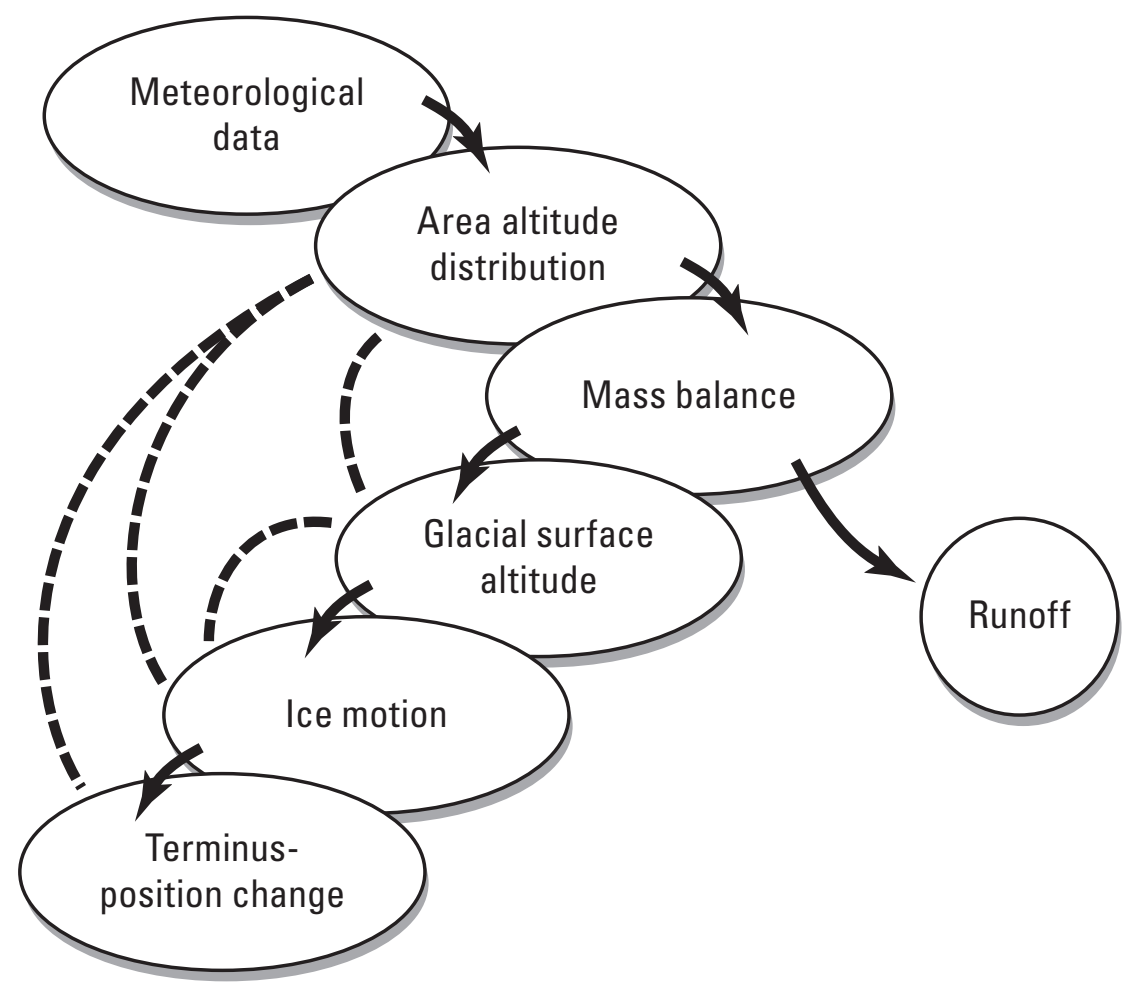

Figure 1. Cause and effect relationships for data presented in this report (modified from Meier, 1965; and Fountain and others, 1997).

Program results are documented in numerous reports: Meier and others (1971) and Tangborn and others (1977) give detailed results for 1966 and 1967, respectively. The series of balance estimates for 1966-77 are reported by Meier and others (1980). Since 1966, the Gulkana Glacier data set has been published by the World Glacier Monitoring Service (http://www.geo.unizh.ch/wgms/) in a 5-year Fluctuations of Glaciers series (Kasser, 1967; Muller, 1977; Haeberli, 1985; Haeberli and Müller, 1988; Haeberli and Hoelzle, 1993; Haeberli and others, 1998) and in a 2-year Glacier Mass Balance Bulletin series (Haeberli and Herren, 1991; Haeberli and others, 1993, 1994, 1996, 1999). March and Trabant (1996, 1997) and March (1998, 2000, 2003) describe the program for intervals between 1992 and 1996. Trabant and March (1999) published the basic balance series through 1998. Select references, data, maps, and photographs from Gulkana Glacier are available at http://ak.water.usgs.gov/glaciology/.

Interpretations of regional climate-glacier relations using Gulkana Glacier data include papers by Fahl (1973), Letréguilly and Reynaud (1989), Walters and Meier (1989), Dowdeswell and others (1997), Hodge and others (1998), Trabant and others (1998), Bitz and Battisti (1999), Elsberg and others (2001), Rasmussen and Conway (2004), and Josberger and others (2006, 2007). The validity of long-term cumulative mass balance at Gulkana Glacier has been verified recently by a comparison of the geodetic (photogrammetry) and glaciological mass-balance techniques in Cox (2002), and Cox and March (2004).

\section{Theory}

Classical glaciological mass balance methodology is well described in the literature, including concise theoretical descriptions (e.g. Mayo and others, 1972; Patterson, 1994) and glaciological field methods (Østrem and Brugman, 1991). We give a minimal framework for understanding the measurements and interpretation presented herein. We follow the terminology established by Mayo and others (1972), for the combined mass balance system, which relates point measurements to time-transgressive stratigraphic horizons, or summer surfaces.

Mass balance is measured at markers drilled into the glacier surface at predetermined locations (index sites), then extrapolated over the entire glacier area. We refer to point measurements as local, or site balances, $b$, representing glacier thickness changes at a marker in water equivalent (weq) units. Mass is lost from the glacier system through ablation $(b<0)$ and subsequent runoff. Ablation can occur at the surface from climatic forcing (old firn and ice melt, $b(i)$ ) 
or from frictional, strain or geothermal heat transfer at the bed or by water transport, which we refer to as internal ablation $(b(j))$. Mass is gained $(b>0)$ through snowfall and wind and avalanche transport $(b(f))$ or by refreezing of meltwater below the summer surface in old firn $(b(k))$, which is called internal accumulation. Seasonal measurement intervals provide enough information to evaluate net, seasonal, and annual mass budgets.

Net balance, $b_{n}$, is the change in snow, firn, and ice storage between times of minimum mass and is not referenced to fixed dates. Direct stake, pit, core, and probe measurements give the net balance at each index site. The timing of this mass minimum occurs asynchronously on different parts of the glacier (earlier high on the glacier and later low on the glacier, as summer melting usually ends earlier on the upper glacier than the lower glacier). Integrating local balance minimums over the glacier area, as is done by some researchers, yields a close approximation to the glacier average net balance, but unlike the true area-averaged, net balance it is not defined with regard to time and does not represent the actual balance on the glacier at any moment. Here we take the extra step to calculate the true glacier averaged net balance that is defined in time and which represents the actual balance on the glacier. Interpretations of net balance often are generalized conceptually to represent the changes for a glacier on a so-called 'yearly' basis even though the net balance period is different each year and may differ substantially from a full year.

Mayo and others (1972) defined winter balance as changes in mass from minimum mass to maximum mass in a season. Measured winter balance, $b_{m}(s)$, is evaluated during late winter or spring, typically mid-April, to quantify the average snow thickness above the previous summer surface near the time of the maximum winter snow balance. This value differs from the maximum winter snow balance, $b_{w}(s)$, which quantifies the maximum snow mass during the balance year and may occur either before or after the measured winter snow balance. All values may be evaluated as glacier-wide averages as denoted with a bar as in $\bar{b}_{m}(s)$. The World Glacier

Monitoring Service (WGMS) publishes a winter balance that is not identified clearly as any of these and may vary from glacier to glacier. The glacier-wide averaged measured winter snow balance, $\bar{b}_{m}(s)$, is probably the most common winter balance value reported by WGMS.

Summer balance, $b_{s}$, was defined by UNESCO (1970) as the mass loss from the maximum mass state to minimum mass state in a given season. The summer balance can be determined from difference between the maximum winter balance, $b_{w}(s)$, and the net (yearly) balance, $b_{n}$.

$$
b_{s}=b_{n}-b_{w}(s) \text {. }
$$

Annual mass balance, $b_{a}$, is the change in snow, firn, and ice storage for the balance year (same as water year), the period between October 1 of any year and September 30 of the following year. Evaluation of the annual balance requires model estimates of initial snow balance, $b_{0}(s)$, and initial ice balance, $b_{0}(i)$, at the beginning of the water year and the final late snow balance, $b_{1}(l s)$, and final ice balance, $b_{1}(i)$, at the end of the water year for each index site (Mayo and others, 1972):

$$
b_{a}=b_{n}-b_{0}(s)+b_{0}(i)+b_{1}(l s)-b_{1}(i) .
$$

Regardless of the measurement time interval (summer, winter, annual or net), local mass balances allow determination of glacier-wide mass balance, $B$, by integrating over the glacier surface area, $A$ :

$$
B=\int b d A
$$

A more useful value for comparison between glaciers is obtained by scaling by the glacier surface area, for an areaaverage balance:

$$
\bar{b}=\int \frac{b d A}{A} .
$$

This number represents an average thickness change in meters water equivalent (mweq) for the glacier allowing direct comparison between the changes of glaciers with different areas. Care must be taken, however, because for some purposes using of the volumetric balance will give the correct result and using area-average balance will give a different and incorrect result.

In practice, we often use a description of mass balance as a function of altitude, $b(z)$ so that interpolated values can be scaled by the map-derived, area altitude distribution, $\gamma(\mathrm{z})$. Integrating this product over the glacier altitude range yields the conventional glacier-wide average balance, which can also be scaled by the glacier area:

$$
\bar{b}=\int b(z) \frac{\gamma(z)}{A} d z
$$

Equation 5 can be corrected for glacier-averaged values of internal ablation:-

$$
\bar{b}=\int b(z) \frac{\gamma(z)}{A} d z+\bar{b}(j)
$$

In practice, equation 5 is estimated by integrating a stepwise-constant balance function. Values of area and balance are measured or interpolated at regular elevation 
intervals (often $50-100 \mathrm{~m}$ ) depending on the size of glacier and measurement density. For each interval, the contribution to the integral is the product of the median altitude balance (interpolated from the balance-altitude distribution) and the fraction of the total glacier area within the interval.

Cumulative balances, $\beta$, are estimated by summing glacier-wide balances over balance years $i=1,2, \ldots, N$ :

$$
\beta=\sum_{i=1}^{N} B_{i}
$$

to describe whether a glacier is growing $(\beta>0)$ or shrinking $(\beta<0)$ in time.

When glacier-climate interactions are the focus of the program, the reference surface balance (Elsberg and others, 2001 ) is calculated, where the glacier geometry is fixed in its initial state (Elsberg and others, 2001; Harrison and others, 2005). If the hydrologic budget is the focus of the measurement program, the conventional mass balance should be calculated which requires an updated geometry (surface area and area-altitude distribution) each year. Comparison of the two balances can highlight glacier dynamics (Harrison and others, 2009). Although the theory of the reference surface balance is relatively new in glaciology, it has actually been calculated unknowingly far more often than conventional balance due to the time consuming and expensive nature updating the glacier geometry on an annual basis, as is necessary for correctly determining conventional balance.

\section{Gulkana Glacier Basin and its Climate}

Gulkana Glacier (figs. 2 and $\underline{3}$; $63^{\circ} 16^{\prime} \mathrm{N}, 145^{\circ} 25^{\prime} \mathrm{W}$ ) is located in a $31.5-\mathrm{km}^{2}$ basin on the south flank of the eastern Alaska Range. This basin is drained by Phelan Creek. The accumulation area consists of four adjacent cirques with east, south, and west exposures that reach altitudes as high as $2,470 \mathrm{~m}$. About 60 percent of the glacier is accumulation area. The 1966-2001 average equilibrium line altitude (ELA) is $1,770 \mathrm{~m}$, about $50 \mathrm{~m}$ higher than the expected ELA in steady state. The ablation area flows south-southwestward, and the

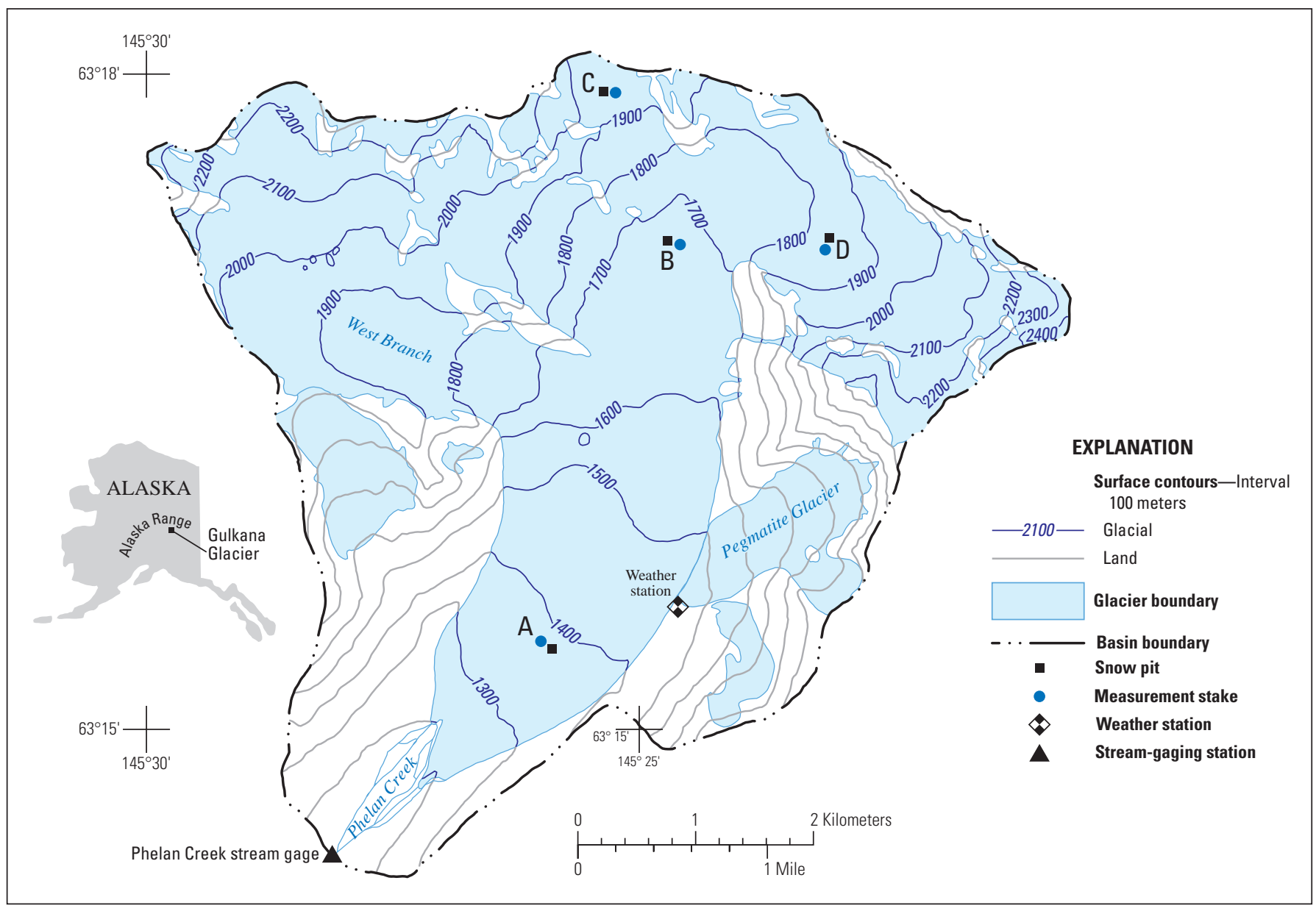

Figure 2. Index measurement sites $A, B$, and $D$, glacier surface altitudes, weather and stream-gaging stations, and boundary of Gulkana Glacier, Alaska (horizontal datum, NAD 27; vertical datum, NGVD 1929). 
terminus is lightly covered with rock debris (fig. 3) below $1,160 \mathrm{~m}$. Slightly contorted moraines near the terminus (fig. 3) suggest that Gulkana Glacier may have surged in the past, but no flow instabilities have been detected since the early 1960s. The most recent period of advance ended around 1900 with the termination of the Little Ice Age (LIA; Péwé and Reger, 1983). Total recession since then is about $3 \mathrm{~km}$. During our monitoring program the glacierized surface area of the $31.5 \mathrm{~km}^{2}$ basin decreased about 20 percent from $19.5 \mathrm{~km}^{2}$ to less than $17 \mathrm{~km}^{2}$.

The climate in this part of Alaska is best described as continental. Daily mean temperatures display large variability, ranging from $-35^{\circ} \mathrm{C}$ to $15^{\circ} \mathrm{C}$. The mean annual air temperature at the weather station adjacent to the middle of the glacier's ablation zone is $-3^{\circ} \mathrm{C}$; at the ELA the mean annual air temperature is $-5^{\circ} \mathrm{C}$ assuming a lapse rate of $-6.6^{\circ} \mathrm{C} / \mathrm{km}$ (air cools with increasing altitude). The average annual precipitation gage catch at the weather station is about 1 mweq. Catch efficiency of the gage averages about 60 percent, so annual average precipitation is about $1.6 \mathrm{~m}$. The basin average runoff since the measurement program began is $1.9 \mathrm{~m}$ indicating that runoff exceeds precipitation by about 16 percent as a result of the glacier losing mass.

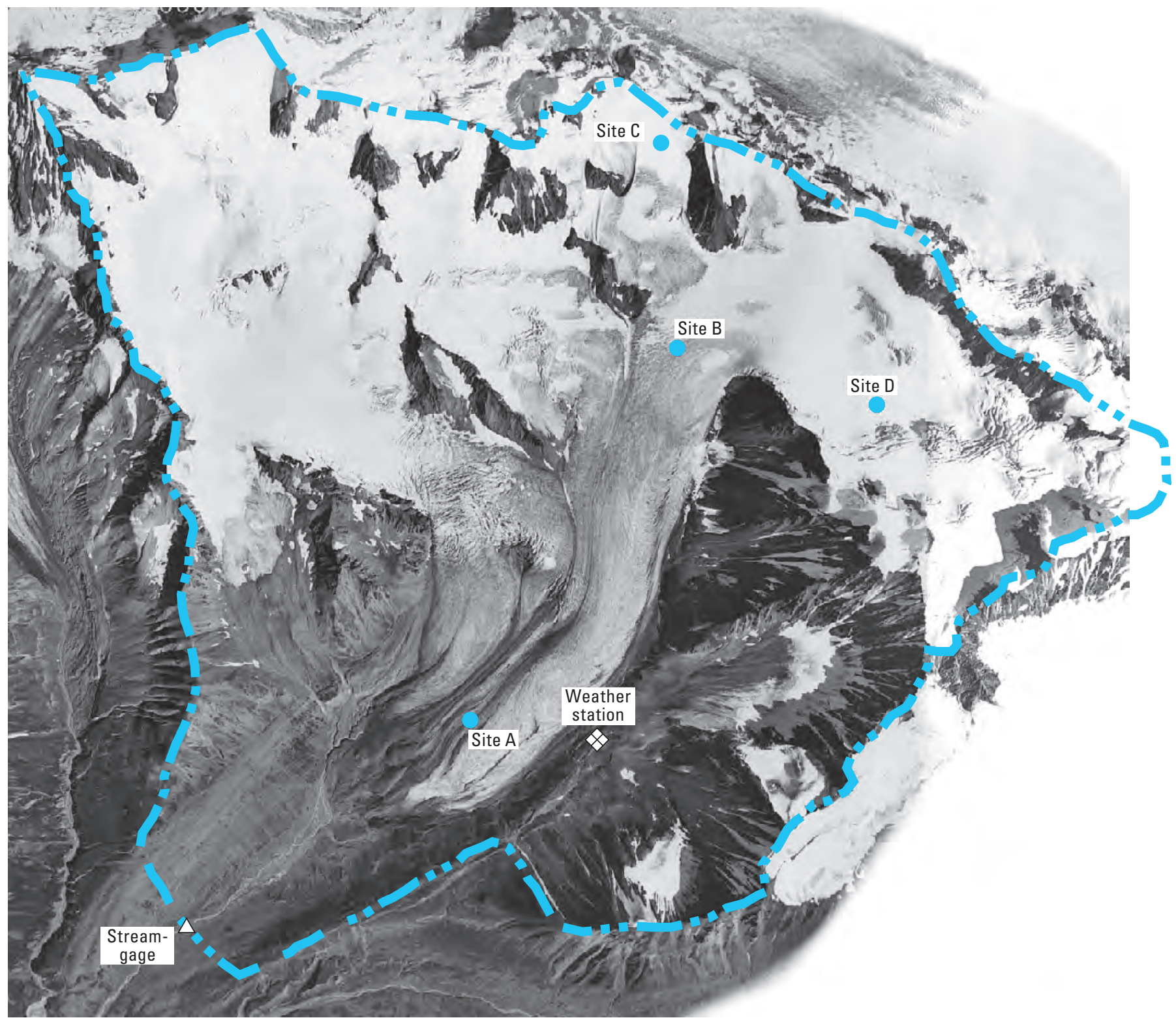

Figure 3. Index measurement sites, stream-gaging station, weather station, and basin boundary, Gulkana Glacier, Alaska, July 11,1993 (photographs Gulkana GI 1 no. 5 and Gulkana GI 2 no. 4 by AeroMetric, Inc.). A, B, and D are index measurement sites for mass balance, ice motion, and surface altitude. Dash-dot-dot-dash line indicates basin boundary. 


\section{Methods}

The core of the Gulkana Glacier monitoring program consists of surface mass balance, surface altitude, and ice motion. Measurements were made at three fixed index sites (A, B, and C or D; figs. 2 and $\underline{3}$, table 1). Sites A and B are in the ablation zone; site $\mathrm{B}$ is the higher of the two and is near the ELA; site C and D are in the accumulation zone. Site C was used as the high site through 1975; Site D has been used as the high site since then. Streamflow and weather observations are critical to interpret the mass balance measurements with respect to basin-wide water balance and climate. Weather observations also are critical in accurately extrapolating endof-season point values from the time of seasonal observations, which seldom coincide exactly with the end of each season.

\section{Glacier Mass Balance}

We provide data using the terminology described above, largely following Mayo and others (1972). We include point estimates of internal accumulation as outlined in Trabant and Mayo (1985) and glacier-average corrections for internal ablation as described by March and Trabant (1997). Our results include estimates of net (stratigraphic) and annual (fixed-date) balances as well as modeled adjustment parameters required for these calculations.

Stake observations are made in early spring and late autumn in attempts to coincide with mass balance extremes while facilitating fieldwork. In addition to measuring the near-maximum balance, observations during the spring add redundancy to summer surface measurements made during the previous autumn. Because little or no melt of the summer surface occurs between these two measurements, the stake height of the surface should be the same. This height is seldom the same, however, because of variable snow depths, surface roughness, and measurement error; hence, the redundancy supports an assessment of stake-measurement errors.

\section{Site Balances}

Although mass-balance measurement locations commonly are referred to as points on a glacier, they are treated as small areas $25-75 \mathrm{~m}$ in radius, centered on each index site. Snow depth measurements are made throughout this area to average out surface irregularities. Likewise, stake readings are made to best represent the intersection of the average glacier surface with the stake.

Stake positions were adjusted to correct for lean and bending $\left(b^{\prime}\right)$, which are common after the initial near-vertical installation of the stakes (March, 2000). These corrections also improve the precision of ice motion estimates. When possible, we make redundant stake measurements; although this often delays assignment of final balance values by one or two seasons, it averages local surface roughness and reduces balance errors (March, 2000).

Temporal extrapolations between measurements are necessary to estimate balances such as the maximum winter balance, glacier-averaged net balance, and annual balance. We used a two-parameter linear model that relates the air temperature and precipitation catch recorded at the weather station to each local site balance. The temperature was lapsed from the recorder altitude to each of the index-site altitudes using the wet-adiabatic lapse rate of $-6.6^{\circ} \mathrm{C} / \mathrm{km}$. Model ablation rates ranged between $3.5 \mathrm{~mm}$ and $5 \mathrm{~mm}$ water equivalent per positive degree day for snow and twice that when the surface was ice or firn. (Positive degree days is the sum of average daily air temperatures that are above freezing.) This range of values agrees closely with those common in the literature (Braithwaite and Olesen, 1985, 1993; Braithwaite, 1995; Jóhannesson and others, 1995). Modeled accumulation rates are 1.5-4.0 times the precipitation catch for lapsed temperature below $1.8^{\circ} \mathrm{C}$.

Table 1. Coordinates of index sites $A, B, C$, and D.

[UTM, Universal Transverse Mercator projection. X, Y, Easting, Northing in meters; latitude, longitude in decimal degrees. Abbreviations: NAD 83, North American Datum 1983; m, meter; dec.deg., decimal degrees]

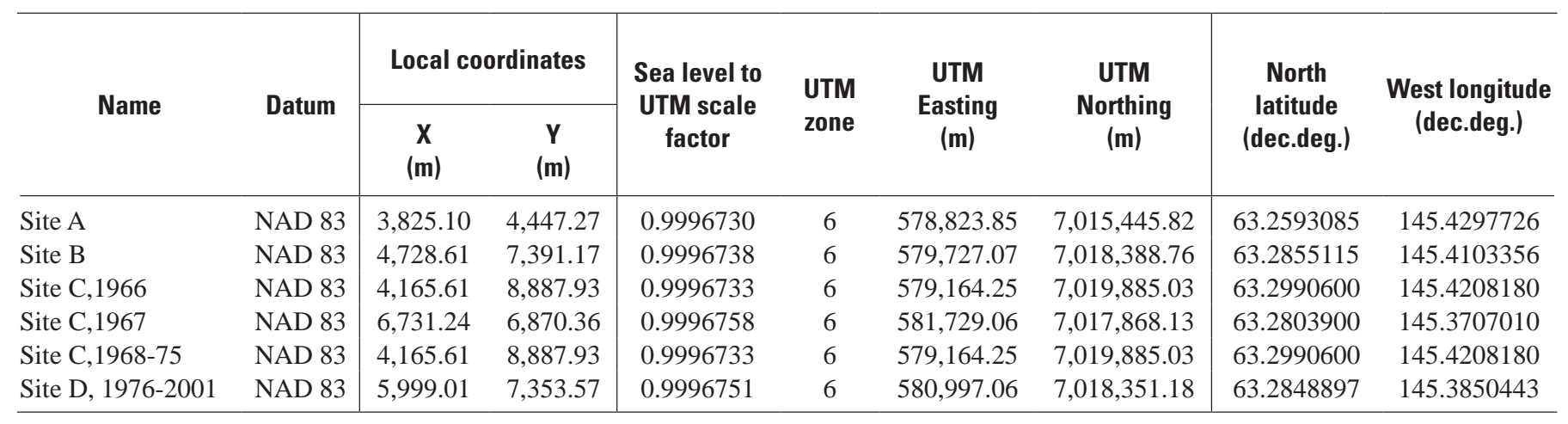


Ablation rate and the precipitation-catch multipliers are not fixed in this model. Separate values are determined for each measurement period at each index site to equate simulated and measured balances. Thus, the model serves only to distribute measured balances over each measurement interval enabling estimates of annual and net balances. During the autumn-to-spring measurement period, no ablation usually occurs, so the model is reduced to a one-parameter model dependent only on precipitation. During the spring-to-autumn measurement period, little or no accumulation occurs, so the model again is reduced to a one-parameter model dependent only on temperature. Small quantities of accumulation early in the spring-to-autumn measurement period are modeled using the precipitation-catch multiplier determined for the previous winter. Small quantities of accumulation near the end of the spring-to-autumn measurement period are modeled by adjusting a separate precipitation-catch multiplier until the measured late snow balance is matched. These calculated site balances differ from the tabulated net balances by small increments, $b_{n 0}$ and $b_{n \mathrm{l}}$.

\section{Area-Averaged Balances}

Although mass balance is measured at only three sites at Gulkana Glacier, March and Trabant (1996) showed that the balance gradient is strongly nonlinear. Therefore, the concern that the limited number of index sites introduces substantial error in glacier-wide balance is warranted. For this reason, the index site concept has undergone rigorous scrutiny. Extensive balance networks measured in 1966 and 1967 were used to select a reduced site distribution (March and Trabant, 1996). We purposefully structured the program with only three altitude intervals whose boundaries are exactly one-half way between the index sites. With this system at Gulkana Glacier, the average balance value in each altitude interval equals the measured site balance value. No interpolation or extrapolation was necessary, and site measurements become the average balance for each altitude interval. No guarantee exists that this approach will work on other glaciers. For added security, we compared our mass balance estimates (called glaciological balances) to geodetic (photogrammetric) balance estimates. The two types of estimates agreed at the $1-\mathrm{m}$ level and the glaciological estimates did not have systematic error propagation issues (Cox and March, 2004). Over the duration of the program, we determined the error in the yearly glaciological glacier-averaged balances to be $\pm 0.2 \mathrm{~m}$ (March and Trabant, 1996). The current index sites may not yield high-quality results indefinitely, however, and continued checks between the glaciological and geodetic balances are essential.
In estimates of glacier-averaged mass balance, we include glacier-averaged internal ablation, $b(j)$. This term is derived from three internal and subglacial energy sources: (1) the geothermal heat at the bed of the glacier, (2) the potential energy loss from ice motion, and (3) the potential energy loss from water flowing through the glacier and along the bed of the glacier (Mayo, 1992; March and Trabant, 1997). These terms are quite small annually, but if neglected, would accumulate as a bias error over time.

\section{Equilibrium Line Altitude and Accumulation Area Ratio}

The ELA is the average altitude where the net mass balance is zero and separates the accumulation area from the ablation area (where more material is lost during the year through ablation than gained by accumulation). Sometimes the ELA corresponds to the highest transient snow or firn line reached in the melt season, but the ELA commonly is lower on the glacier because of internal accumulation in firn or the presence of superimposed ice. When superimposed ice is exposed at the surface, its lower boundary defines the ELA. Superimposed ice may form in the spring as snowmelt percolates down to the impermeable and cold old ice surface and refreezes, and is considered as part of the current year's snow. At Gulkana Glacier, the ELA can be extremely complicated, seldom crossing the glacier along a single altitude contour. Accurate estimation requires well-timed vertical or high-angle oblique aerial photography over the whole glacier, a rare occurrence. Instead, for reliability, linear interpolation from the balance-altitude curve of the three index sites is used to estimate the ELA.

The accumulation area ratio (AAR) is the fraction of the glacier area over which accumulation or net mass gain occurs. It includes areas of new firn, superimposed ice, and areas of old firn below the snowline where internal accumulation exceeds old firn loss.

\section{Glacier Geometry}

\section{Area Altitude Distribution}

The area altitude distribution (AAD) most completely describes the history of glacier geometry. An original AAD from 1967 has been used to calculate and report all glacieraveraged balances prior to this publication. As such, previous balances have not been conventional net mass balances, but have been reference surface net balances (Elsberg and others, 2001). 
For the first time, we are reporting the time varying AAD for Gulkana Glacier (fig. 4, table 2), so that a true hydrologic balance can be estimated. Four new AADs are introduced (1900, 1974, 1993, and 1999), and from the five complete descriptions of AAD we estimate an annual value using linear interpolation or extrapolation. We estimate an AAD for the Little Ice Age (LIA) maximum, circa 1900, by using the LIA trim lines as the glacier edge and preserving the modern surface curvature. At about the modern ELA, where trim lines disappear, the paleosurface was "feathered" into the 1974 surface and the LIA surface is estimated to be the same as the 1974 surface above the ELA. The original 1967 AAD (Tangborn and others, 1977) is based on a 100-m contour topographic map produced by examination of the USGS 1950s aerial photography and resultant 1:63,360-scale topographic maps (Mt. Hayes A-3 [1950] and B-3 [1955]), and more recent oblique aerial photography (dates unknown) taken by Austin Post and Larry Mayo (part of the USGS Ice and Climate Project Photography Collection, GeoData Center, Fairbanks, Alaska, available at http://www.gi.alaska. edu/services/geodata/). Higher quality estimates of glacier area derive from photogrammetric analysis of vertical aerial photography acquired September 4, 1974; August 11, 1993; and August 18, 1999 (Cox and March, 2004).

\section{Terminus Position}

The terminus is the fastest changing region of the glacier, and surveying the ice margin provides the best description of changes to the length and lower area of the glacier. We made late-summer, hand-held GPS terminus surveys each year between 1997 and 2001 by walking about $4 \mathrm{~km}$ of the lower glacier margin (fig. 5, tables 3-9). We used a Collins Rockwell Precision Lightweight GPS Receiver, which is a single-frequency (L1) encrypted $\mathrm{P}(\mathrm{Y})$ code receiver capable of Precise Positioning Service (PPS) operation. This receiver provided single-station, mapping grade solutions PPS, prior to the removal of the selective availability encryption in 2000 . Wilson and others (1999) estimated errors of 4.5 and $6 \mathrm{~m}$ in the horizontal and vertical directions, respectively, with improvement to $5 \mathrm{~m}$ vertical resolution after 1999. Our own assessment of the errors showed repeat capability averaging $3 \mathrm{~m}$ at a known benchmark (Péwé; fig. 5). Software-derived error estimates averaged 5-6 $\mathrm{m}$ (5 percent greater than $10 \mathrm{~m}$; maximum error $20 \mathrm{~m}$ ) for about 180 terminus locations surveyed in 1999 and 2000; thus, an average error in the terminus data of $6 \mathrm{~m}$ was assumed.

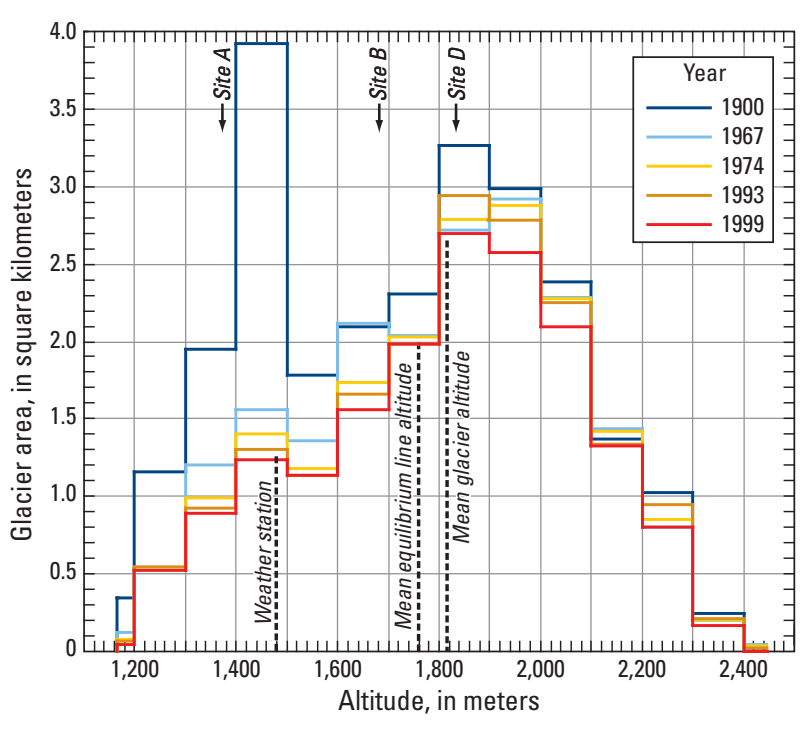

Figure 4. Area altitude distribution of Gulkana Glacier, Alaska, in 1900, 1967, 1974, 1993, and 1999 for 100 -meter altitude bins. Altitudes of index sites, the weather station, the mean equilibrium line, and mean altitude of the glacier are shown.

A coarse optical survey from 1985 and photogrammetrically derived terminus locations from vertical aerial photography acquired July 11, 1993 predate GPS measurements (fig. 5, table 4). We estimated the accuracy of optically surveyed positions at $0.1 \mathrm{~m}$ and photogrammetrically derived positions at less than $1 \mathrm{~m}$. However, debris covers much of the terminus (fig. 3), and the transition from clean glacier ice to debris-covered ice to ice-cored moraine and till is gradual and indistinct, adding many meters of uncertainty to the terminus definition in some areas, regardless of survey method. This uncertainty is largest along a $0.5-\mathrm{km}$ stretch of the southwest terminus where the error may be as much as $200 \mathrm{~m}$. 


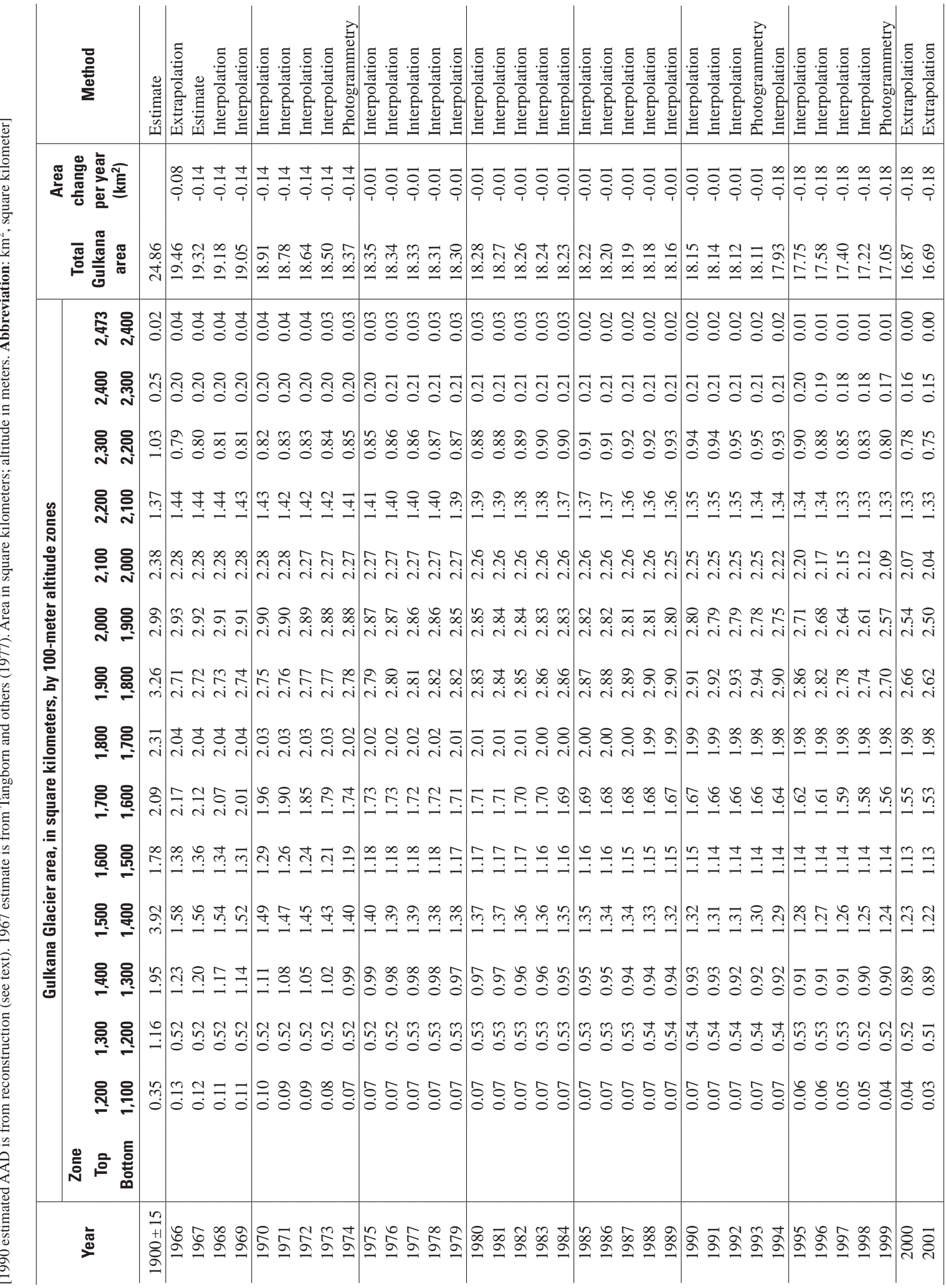




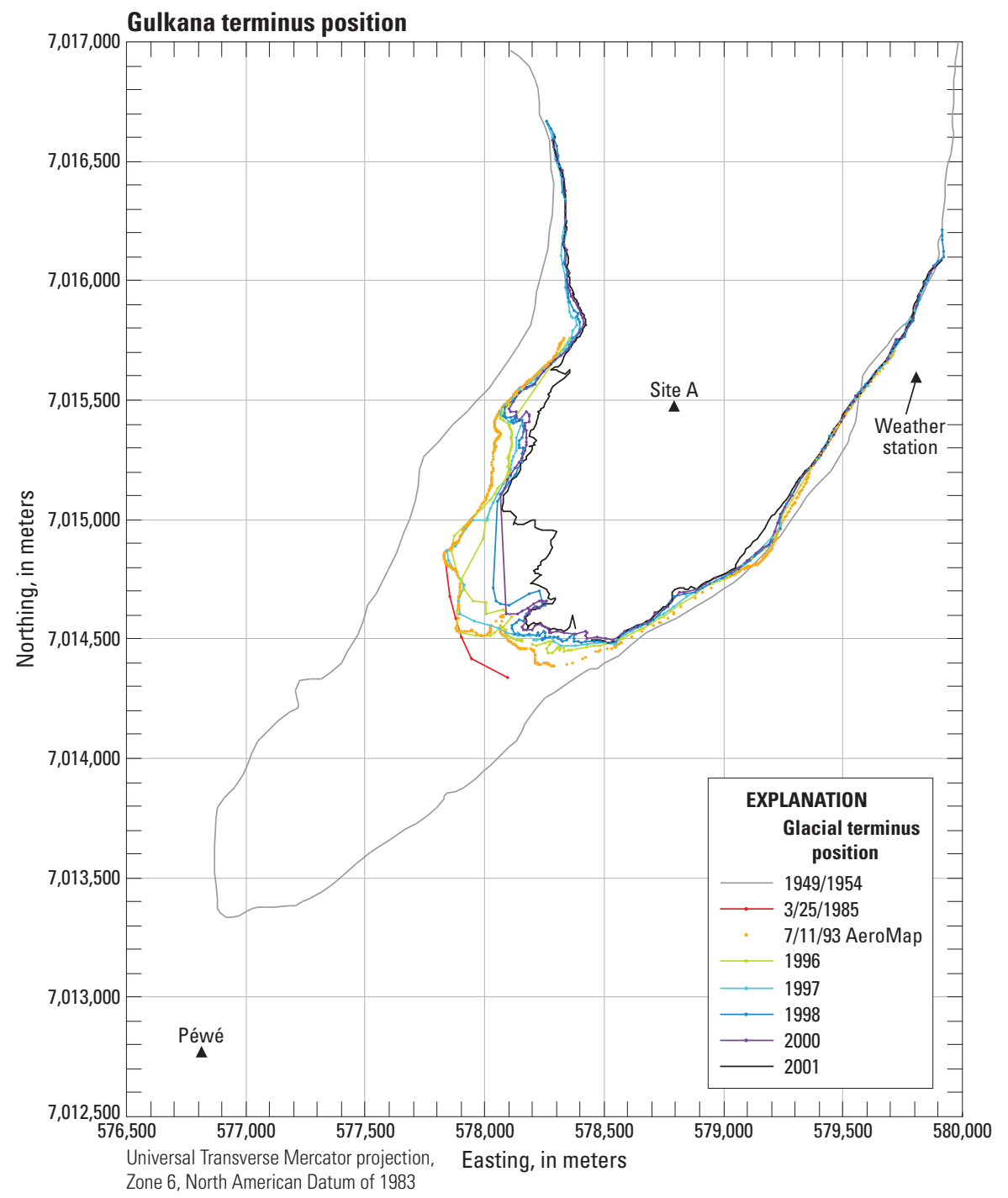

Figure 5. Glacier terminus positions from Global Positioning System surveys at Gulkana Glacier, Alaska, in 1996 (previously published in March [2003]), 1997, 1998, 1999, 2000, and 2001. From U.S. Geological Survey 1:63,360-scale Mt. Hayes A-3 quadrangle (1949 photography; area south of about 7,014,200 m Northing) and B-3 quadrangle (1954 photography; area north of about 7,014,200 $\mathrm{m}$ Northing) digital raster graphic (DRG) files; from optical survey in 1985 and photogrammetry in 1993

Table 3. Optical surveys of terminus position, Gulkana Glacier, March 25, 1985.

[Abbreviations: UTM, Universal Transverse Mercator; NAD 83, North American Datum of 1983; NGVD 29, National Geodetic Vertical Datum of 1929]

\begin{tabular}{cccc}
\hline \multicolumn{2}{c}{ UTM (zone 6) - NAD 83 } & & NGVD 29 \\
\cline { 1 - 2 } Easting & Northing & Altitude \\
\hline \multicolumn{3}{c}{ (meters) } & (meters) \\
\hline 577,833 & Terminus positions \\
577,854 & $7,014,822$ & \\
577,881 & $7,014,676$ & & 1,195 \\
577,900 & $7,014,588$ & & 1,180 \\
577,947 & $7,014,511$ & \\
578,096 & $7,014,415$ & & 1,166 \\
& $7,014,341$ & \\
\hline
\end{tabular}


Table 4. Photogrametric surveys of terminus positions, Gulkana Glacier, July 11, 1993.

[Universal Transverse Mercator Coordinates (UTM) are in UTM zone 6. m, meters. Easting and northing measurements are in meters and North American Datum of 1983 (NAD 83)]

\begin{tabular}{|c|c|c|c|c|c|c|c|c|c|c|c|}
\hline Easting & Northing & Easting & Northing & Easting & Northing & Easting & Northing & Easting & Northing & Easting & Northing \\
\hline 579,715 & ,015,704 & 579,349 & $7,015,170$ & 579,136 & $7,014,812$ & 578,548 & $7,014,467$ & 578,105 & $7,014,489$ & 577,888 & $7,014,588$ \\
\hline 579,712 & $7,015,699$ & 579,348 & $7,015,164$ & 579,134 & $7,014,811$ & 578,543 & $7,014,464$ & 78,099 & $7,014,492$ & 577,890 & $7,014,605$ \\
\hline 579,709 & $7,015,696$ & 579,345 & $7,015,157$ & 579,129 & $7,014,809$ & 578,533 & $7,014,461$ & 578,095 & $7,014,495$ & 77,891 & $7,014,621$ \\
\hline 579,708 & $7,015,693$ & 579,341 & $7,015,149$ & 579,124 & $7,014,808$ & 578,515 & $7,014,457$ & 578,090 & $7,014,506$ & 577,892 & $7,014,633$ \\
\hline 579,706 & $7,015,690$ & 579,335 & $7,015,138$ & 579,120 & $7,014,807$ & 578,480 & $7,014,452$ & 578,087 & $7,014,511$ & & 7.014 \\
\hline 579,704 & $7,015,687$ & 579,328 & $7,015,128$ & 579,117 & $7,014,806$ & 578,474 & $7,014,450$ & 578,083 & $7,014,513$ & 90 & $7,014,650$ \\
\hline 579,703 & $7,015,685$ & 579,323 & $7,015,119$ & 579,114 & $7,014,805$ & 578,466 & $7,014,446$ & 578,076 & 7,01 & & 7014 \\
\hline 579,702 & $7,015,683$ & 579,319 & $7,015,114$ & 579,111 & $7,014,804$ & 578,424 & $7,014,424$ & 578,071 & 7,01 & 577 & $7,014,666$ \\
\hline 579,701 & $7,015,681$ & 579,317 & $7,015,109$ & 579,106 & $7,014,803$ & 578,409 & $7,014,416$ & 578,069 & 7,014 & & $7,014,671$ \\
\hline 579,699 & $7,015,679$ & 579,315 & $7,015,104$ & 579,102 & $7,014,802$ & 578,371 & $7,014,401$ & 578,067 & $7,014,529$ & 577,892 & $7,014,680$ \\
\hline 579,696 & $7,015,675$ & 579,311 & $7,015,095$ & 579,098 & $7,014,801$ & 578,344 & $7,014,394$ & 578,065 & $7,014,536$ & & $7,014,692$ \\
\hline 579,692 & $7,015,668$ & 579,307 & $7,015,086$ & 579,096 & $7,014,800$ & 578,290 & $7,014,385$ & 578,065 & $7,014,543$ & 577,898 & $7,014,703$ \\
\hline 579,685 & $7,015,660$ & 579,301 & $7,015,071$ & 579,094 & $7,014,799$ & & $7,014,385$ & 578,065 & $7,014,551$ & & $7,014,711$ \\
\hline 579,677 & $7,015,649$ & 579,301 & $7,015,071$ & 579,090 & $7,014,797$ & 578,283 & $7,014,386$ & 578,068 & $7,014,559$ & 98 & $7,014,720$ \\
\hline 579,666 & $7,015,636$ & & $7,015,070$ & 579,084 & $7,014,794$ & & $7,014,389$ & & 7,01 & & \\
\hline 579,656 & $7,015,622$ & 579,299 & 7,01 & 579,078 & $7,014,791$ & 71 & 7,01 & 578,077 & 7,01 & & 7,01 \\
\hline 579,645 & $7,015,608$ & 579,295 & 063 & 579,072 & $7,014,788$ & & 7,014 & & 595 & & \\
\hline 579,633 & $7,015,594$ & 579,290 & $7,015,056$ & 579,068 & $7,014,786$ & 578 , & 7,014 & 578,076 & $7,014,596$ & 577 & 7,01 \\
\hline 579,620 & $7,015,578$ & 579,282 & $7,015,047$ & 579,066 & $7,014,785$ & & $7,014,397$ & & 7,01 & & \\
\hline 579,604 & $7,015,561$ & 579,275 & 7,01 & 579,065 & $7,014,785$ & 57 & 7,014 & 578 & 7,01 & 79 & 7,01 \\
\hline & $7,015,541$ & 579,267 & $7,015,027$ & 579,064 & $7,014,784$ & & & & & & \\
\hline 579,571 & 7,01 & 61 & 7,0 & 57 & 7,0 & & 7,0 & & & & 7,0 \\
\hline & 7,01 & & & & & & & & & & \\
\hline 579,542 & 7,01 & 51 & 7,0 & 57 & $7,014,778$ & & 7,0 & & & & 7,01 \\
\hline & & & & & & & $7,014,396$ & & & & \\
\hline 527 & 7,01 & & 7,0 & 57 & 7,0 & & 97 & & 7,0 & & 7,0 \\
\hline 579,523 & $7,015,456$ & 235 & $7,014,956$ & 579,040 & $7,014,768$ & & 7,014 & 4 & $7,014,536$ & 55 & $7,014,795$ \\
\hline 579,518 & $7,015,450$ & 28 & 7,01 & 579,038 & $7,014,767$ & 578 & 7,01 & 25 & 7,01 & & 7,01 \\
\hline 579,511 & $7,015,440$ & 579,222 & $7,014,929$ & 579,034 & $7,014,765$ & 57 & 7,014 & 4 & 7,014 & 57 & $7,014,799$ \\
\hline 579,502 & $7,015,428$ & 19 & 923 & 579,030 & $7,014,763$ & 10 & 411 & 23 & 7,0 & & ,802 \\
\hline 579,493 & $7,015,415$ & 18 & 7,01 &, 024 & $7,014,760$ & & & 8 & & & 7,0 \\
\hline 579,485 & $7,015,403$ & 216 & 7,01 & 579,016 & $7,014,757$ & 10 & 429 & 09 & 7,0 & 57 & 7,01 \\
\hline 579,478 & $7,015,393$ & 9,212 & $7,014,906$ & 579,004 & & & 7,0 & & 7,01 & 57 & 7,0 \\
\hline 579,473 & $7,015,386$ & 579,209 & $7,014,896$ & 579,002 & $7,014,750$ & 578,207 & $7,014,445$ & 577,990 & $7,014,516$ & 577,839 & $7,014,816$ \\
\hline 579,470 & $7,015,381$ & 579,205 & $7,014,887$ & 578,987 & $7,014,743$ & 578,206 & $7,014,449$ & 577,9 & $7,014,515$ & 577, & $7,014,818$ \\
\hline 579,468 & $7,015,377$ & 579,202 & $7,014,880$ & 578,946 & $7,014,713$ & 578,205 & $7,014,452$ & 577,979 & $7,014,516$ & 577,837 & $7,014,820$ \\
\hline 579,465 & $7,015,371$ & 579,199 & $7,014,876$ & 578,912 & $7,014,697$ & 578,202 & $7,014,454$ & 577,971 & $7,014,520$ & 577,836 & $7,014,824$ \\
\hline 579,459 & $7,015,360$ & 579,198 & $7,014,873$ & 578,910 & $7,014,695$ & 578,198 & $7,014,456$ & 577,960 & $7,014,529$ & 577,834 & $7,014,830$ \\
\hline 579,452 & $7,015,347$ & 579,196 & $7,014,871$ & 578,880 & $7,014,671$ & 578,195 & $7,014,457$ & 577,952 & $7,014,534$ & 577,833 & $7,014,837$ \\
\hline 579,444 & $7,015,331$ & 579,194 & $7,014,869$ & 578,819 & $7,014,633$ & 578,191 & $7,014,458$ & 577,946 & $7,014,536$ & 577,831 & $7,014,843$ \\
\hline 579,435 & $7,015,315$ & 579,191 & $7,014,865$ & 578,792 & $7,014,610$ & 578,175 & $7,014,459$ & 577,943 & $7,014,535$ & 577,830 & $7,014,846$ \\
\hline 579,426 & $7,015,300$ & 579,187 & $7,014,860$ & 578,790 & $7,014,607$ & 578,171 & $7,014,459$ & 577,937 & $7,014,534$ & 577,830 & $7,014,849$ \\
\hline 579,417 & $7,015,286$ & 579,182 & $7,014,855$ & 578,767 & $7,014,588$ & 578,163 & $7,014,458$ & 577,928 & $7,014,532$ & 577,831 & $7,014,851$ \\
\hline 579,409 & $7,015,273$ & 579,178 & $7,014,850$ & 578,734 & $7,014,570$ & 578,159 & $7,014,457$ & 577,914 & $7,014,530$ & 577,831 & $7,014,855$ \\
\hline 579,400 & $7,015,262$ & 579,176 & $7,014,847$ & 578,731 & $7,014,568$ & 578,154 & $7,014,458$ & 577,903 & $7,014,530$ & 577,832 & $7,014,859$ \\
\hline 579,392 & $7,015,250$ & 579,174 & $7,014,845$ & & $7,014,555$ & & $7,014,458$ & & $7,014,531$ & & $7,014,862$ \\
\hline 579,383 & $7,015,238$ & 579,172 & $7,014,842$ & 578,658 & $7,014,531$ & 578,148 & $7,014,460$ & 577,888 & $7,014,532$ & 577,834 & $7,014,864$ \\
\hline 579,375 & $7,015,226$ & 579,169 & $7,014,838$ & 578,614 & $7,014,509$ & & $7,014,462$ & 577,885 & $7,014,533$ & & $7,014,865$ \\
\hline 579,367 & $7,015,213$ & 579,164 & $7,014,833$ & 578,614 & $7,014,509$ & 578,139 & $7,014,464$ & 577,883 & $7,014,535$ & 577,836 & $7,014,866$ \\
\hline 579,361 & $7,015,201$ & 579,158 & $7,014,827$ & 578,571 & $7,014,486$ & & $7,014,467$ & 577,882 & $7,014,539$ & 577,839 & $7,014,868$ \\
\hline 579,357 & $7,015,190$ & 579,152 & $7,014,822$ & 578,570 & $7,014,485$ & 578,130 & $7,014,471$ & 577,881 & $7,014,542$ & 577,842 & $7,014,869$ \\
\hline 579,353 & $7,015,182$ & 579,146 & $7,014,818$ & 578,559 & $7,014,477$ & 578,125 & $7,014,476$ & 577,881 & $7,014,546$ & 577,845 & $7,014,871$ \\
\hline 579,352 & $7,015,177$ & 579,142 & $7,014,815$ & 578,558 & $7,014,476$ & 578,121 & $7,014,479$ & 577,881 & $7,014,550$ & 577,849 & $7,014,872$ \\
\hline 579,350 & $7,015,173$ & 579,139 & $7,014,813$ & 578,552 & $7,014,469$ & 578,116 & $7,014,483$ & 577,883 & $7,014,559$ & 577,852 & $7,014,874$ \\
\hline
\end{tabular}


Table 4. Photogrametric surveys of terminus positions, Gulkana Glacier, July 11, 1993.—Continued

[Universal Transverse Mercator Coordinates (UTM) are in UTM zone 6. m, meters. Easting and northing measurements are in meters and North American Datum of 1983 (NAD 83)]

\begin{tabular}{|c|c|c|c|c|c|c|c|c|c|c|c|}
\hline Easting & Northing & Easting & Northing & Easting & Northing & Easting & Northing & Easting & Northing & Easting & Northing \\
\hline 577,856 & $7,014,876$ & 577,922 & $7,014,970$ & 578,031 & $7,015,170$ & 578,058 & $7,015,343$ & 578,086 & $7,015,481$ & 578,218 & $7,015,608$ \\
\hline 577,859 & $7,014,879$ & 577,925 & $7,014,973$ & 578,031 & $7,015,174$ & 578,060 & $7,015,346$ & 578,091 & $7,015,485$ & 578,223 & $7,015,612$ \\
\hline 577,861 & $7,014,882$ & 577,927 & $7,014,975$ & 578,031 & $7,015,181$ & 578,062 & $7,015,350$ & 578,093 & $7,015,487$ & & $7,015,619$ \\
\hline 577,863 & $7,014,884$ & 577,928 & $7,014,977$ & 578,032 & $7,015,190$ & 578,063 & $7,015,354$ & 578,095 & $7,015,489$ & 578,234 & $7,015,625$ \\
\hline 577,864 & $7,014,886$ & 577,930 & $7,014,980$ & 578,032 & $7,015,197$ & 578,064 & $7,015,357$ & 578,096 & $7,015,491$ & 578,240 & $7,015,632$ \\
\hline 577,865 & $7,014,888$ & 577,932 & $7,014,984$ & 578,033 & $7,015,203$ & 578,064 & $7,015,360$ & 578,099 & $7,015,495$ & 578,246 & $7,015,638$ \\
\hline 577,866 & $7,014,889$ & 577,936 & $7,014,991$ & 578,033 & $7,015,207$ & 578,063 & $7,015,362$ & 578,102 & $7,015,501$ & 578,252 & $7,015,643$ \\
\hline 577,866 & $7,014,891$ & 577,942 & 7,014,999 & 578,033 & $7,015,215$ & 578,061 & $7,015,364$ & 578,107 & $7,015,507$ & 578,257 & $7,015,648$ \\
\hline 577,867 & $7,014,893$ & 577,947 & $7,015,006$ & 578,032 & $7,015,226$ & 578,059 & $7,015,367$ & 578,113 & $7,015,513$ & 578,263 & $7,015,652$ \\
\hline 577,868 & $7,014,895$ & 577,952 & $7,015,013$ & 578,032 & $7,015,239$ & 578,056 & $7,015,368$ & 578,118 & $7,015,518$ & 578,267 & $7,015,654$ \\
\hline 577,868 & $7,014,896$ & 577,957 & $7,015,018$ & 578,033 & $7,015,254$ & 578,054 & $7,015,370$ & 578,123 & $7,015,523$ & 578,270 & $7,015,656$ \\
\hline 577,868 & $7,014,896$ & 577,962 & $7,015,024$ & 578,034 & $7,015,267$ & 578,052 & $7,015,371$ & 578,127 & $7,015,528$ & 578,272 & $7,015,658$ \\
\hline 577,869 & $7,014,897$ & 577,967 & $7,015,032$ & 578,036 & $7,015,277$ & 578,050 & $7,015,372$ & 578,131 & $7,015,532$ & 578,275 & $7,015,661$ \\
\hline 577,871 & $7,014,897$ & 577,973 & $7,015,042$ & 578,038 & $7,015,285$ & 578,049 & $7,015,372$ & 578,134 & $7,015,536$ & 578,279 & 7,015,666 \\
\hline 577,873 & 7,014,899 & 577,980 & 7,01 & 578,039 & $7,015,290$ & 578,048 & $7,015,374$ & 578,138 & $7,015,540$ & 578,284 & $7,015,671$ \\
\hline 577,875 & $7,014,900$ & 577,987 & $7,015,062$ & 578,040 & $7,015,292$ & 578,046 & $7,015,377$ & 578,142 & $7,015,544$ & 578,288 & $7,015,676$ \\
\hline 577,878 & $7,014,901$ & 577,994 & $7,015,072$ & 578,041 & $7,015,294$ & 578,045 & $7,015,382$ & 578,147 & $7,015,548$ & 578,291 & $7,015,679$ \\
\hline 577,881 & $7,014,902$ & 578,000 & $7,015,079$ & 578,042 & $7,015,297$ & 578,043 & 7,0 & 578,152 & $7,015,552$ & 578,292 & $7,015,681$ \\
\hline 577,884 & $7,014,902$ & 578,003 & $7,015,084$ & 578,043 & $7,015,300$ & 578,042 & $7,015,391$ & 578,158 & 7,015,557 & 578,294 & $7,015,684$ \\
\hline 577,885 & $7,014,903$ & 578,005 & $7,015,087$ & 578,044 & $7,015,302$ & 578,041 & $7,015,396$ & 578,163 & $7,015,561$ & 578,297 & $7,015,689$ \\
\hline 577,887 & $7,014,905$ & 578,006 & $7,015,090$ & 578,044 & $7,015,304$ & 578,041 & $7,015,399$ & 578,168 & $7,015,566$ & 578,300 & $7,015,695$ \\
\hline 577,889 & $7,014,909$ & 578,008 & $7,015,095$ & 578,045 & $7,015,306$ & 578,041 & $7,015,402$ & 578,172 & $7,015,570$ & 578,304 & $7,015,702$ \\
\hline 577,892 & $7,014,915$ & 578,010 & $7,015,101$ & 044 & 7,01 & 41 & & 75 & 73 & 578 & $7,015,711$ \\
\hline 577,895 & $7,014,923$ & 578,013 & $7,015,108$ & 578,044 & $7,015,309$ & 578,041 & $7,015,406$ & 578,177 & $7,015,575$ & 578,309 & $7,015,719$ \\
\hline 577,899 & $7,014,931$ & 578,016 & $7,015,116$ & 578,044 & $7,015,311$ & 578,041 & $7,015,408$ & 578,178 & $7,015,576$ & 578,311 & $7,015,725$ \\
\hline 577,903 & $7,014,940$ & 578,019 & $7,015,123$ & 578,044 & $7,015,313$ & 578,042 & $7,015,412$ & 578,178 & $7,015,576$ & 578,312 & $7,015,729$ \\
\hline 577,906 & $7,014,947$ & 578,022 & $7,015,130$ & 578,043 & $7,015,315$ & 578,044 & $7,015,417$ & 578,179 & $7,015,577$ & 578,313 & $7,015,731$ \\
\hline 577,908 & $7,014,952$ & 578,024 & $7,015,135$ & 578,043 & $7,015,316$ & 578,047 & $7,015,424$ & 578,181 & $7,015,578$ & 578,315 & $7,015,733$ \\
\hline 577,910 & $7,014,956$ & 578,025 & $7,015,138$ & 578,043 & $7,015,31$ & 578,050 & $7,015,430$ & 578,184 & $7,015,580$ & 578,318 & 7,015,737 \\
\hline 577,911 & $7,014,958$ & 578,026 & $7,015,140$ & 578,043 & $7,015,317$ & 578,053 & $7,015,435$ & 578,188 & $7,015,582$ & 578,321 & $7,015,741$ \\
\hline 577,912 & $7,014,959$ & 578,027 & $7,015,142$ & 578,043 & $7,015,319$ & 578,055 & $7,015,439$ & 578,194 & $7,015,586$ & 578,325 & $7,015,747$ \\
\hline 577,913 & $7,014,961$ & 578,027 & $7,015,144$ & 578,044 & $7,015,321$ & 578,056 & $7,015,441$ & 578,199 & $7,015,590$ & 578,329 & $7,015,754$ \\
\hline 577,915 & $7,014,962$ & 578,028 & $7,015,147$ & 578,044 & $7,015,325$ & 578,059 & $7,015,445$ & 578,204 & $7,015,594$ & 578,331 & $7,015,759$ \\
\hline 577,916 & $7,014,964$ & 578,028 & $7,015,152$ & 578,046 & $7,015,330$ & 578,063 & $7,015,452$ & 578,208 & $7,015,598$ & & \\
\hline 577,917 & $7,014,965$ & 578,029 & $7,015,158$ & 578,048 & $7,015,335$ & 578,068 & $7,015,460$ & 578,211 & $7,015,600$ & & \\
\hline 577,918 & $7,014,966$ & 578,030 & $7,015,163$ & 578,051 & $7,015,338$ & 578,074 & $7,015,468$ & 578,213 & $7,015,602$ & & \\
\hline 577,920 & $7,014,968$ & 578,030 & $7,015,167$ & 578,055 & $7,015,341$ & 578,080 & $7,015,475$ & 578,215 & $7,015,604 \|$ & & \\
\hline
\end{tabular}


Table 5. Global Positioning System terminus position surveys, Gulkana Glacier, September 3, 1997.

[Universal Transverse Mercator (UTM) coordinates are in UTM zone 6. Easting and northing measurements are in meters and NAD 83, North American Datum of 1983; NGVD 29, National Geodetic Vertical Datum of 1929]

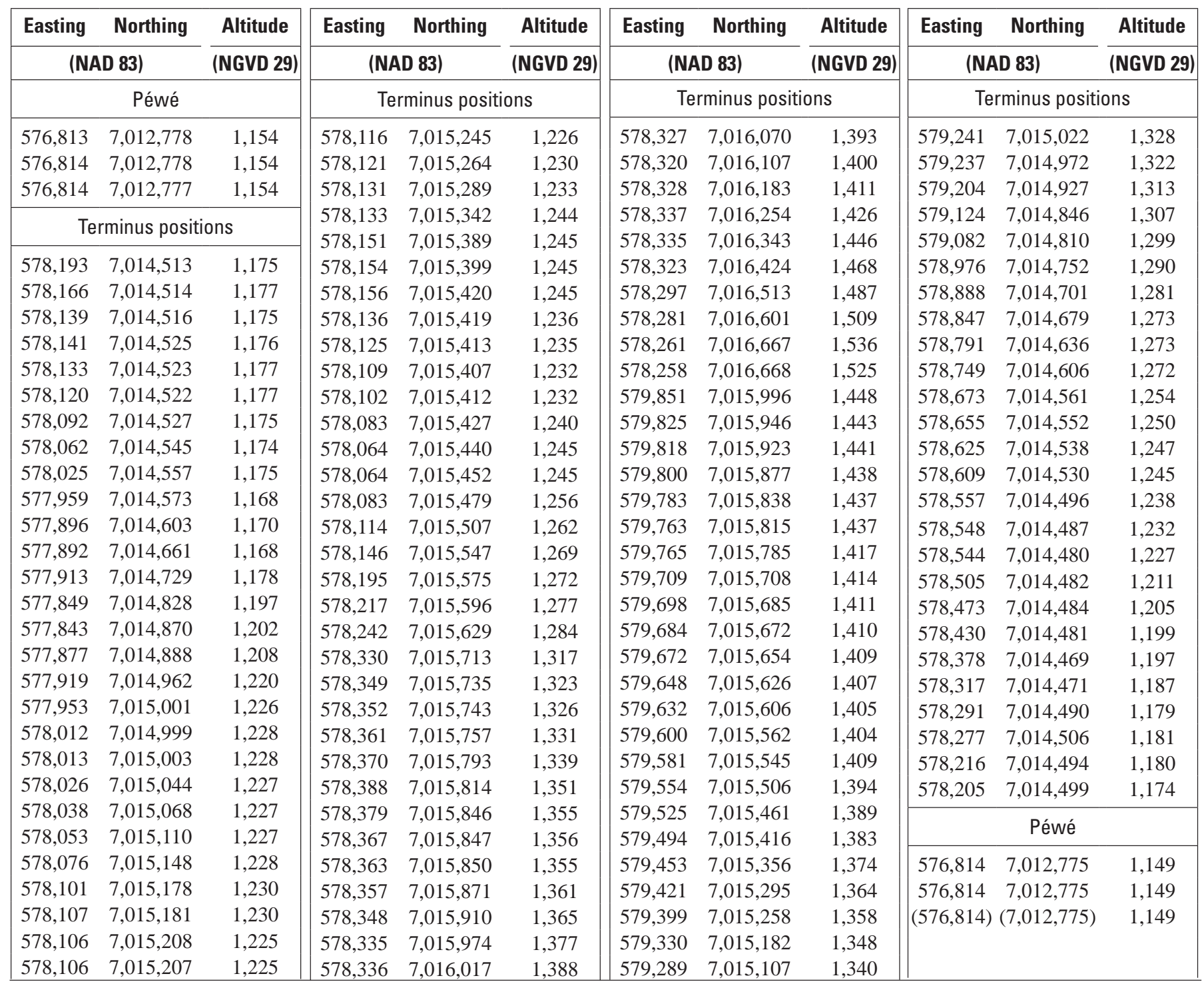


Table 6. Global Positioning System terminus position surveys, Gulkana Glacier, September 9, 1998.

[Universal Transverse Mercator (UTM) coordinates are in UTM zone 6. Easting and northing measurements are in meters. NAD 83, North American Datum of 1983; NGVD 29, National Geodetic Vertical Datum of 1929]

\begin{tabular}{|c|c|c|c|c|c|c|c|c|c|c|c|}
\hline Easting & Northing & Altitude & Easting & Northing & Altitude & Easting & Northing & Altitude & Easting & Northing & Altitude \\
\hline \multicolumn{2}{|c|}{ (NAD 83) } & (NGVD 29) & \multicolumn{2}{|c|}{ (NAD 83) } & (NGVD 29) & \multicolumn{2}{|c|}{ (NAD 83) } & (NGVD 29) & \multicolumn{2}{|c|}{ (NAD 83) } & (NGVD 29) \\
\hline \multicolumn{3}{|c|}{ Péwé } & \multicolumn{3}{|c|}{ Terminus positions } & \multicolumn{3}{|c|}{ Terminus positions } & \multicolumn{3}{|c|}{ Terminus positions } \\
\hline 576,817 & $7,012,767$ & 1,150 & 578,135 & $7,015,410$ & 1,248 & 578,308 & $7,016,496$ & 1,497 & 579,231 & 7,014,985 & 1,317 \\
\hline 576,816 & 7,012,765 & 1,152 & 578,120 & $7,015,406$ & 1,242 & 578,306 & $7,016,523$ & 1,503 & 579,236 & $7,014,961$ & 1,324 \\
\hline 576,815 & $7,012,765$ & 1,154 & 578,115 & $7,015,410$ & 1,245 & 578,300 & $7,016,563$ & 1,512 & 579,201 & $7,014,910$ & 1,316 \\
\hline \multirow{2}{*}{\multicolumn{3}{|c|}{ Terminus positions }} & 578,113 & 7,015,415 & 1,246 & 578,288 & 7,016,595 & 1,516 & 579,184 & 7,014,888 & 1,313 \\
\hline & & & 578,127 & $7,015,424$ & 1,251 & 578,288 & $7,016,612$ & 1,521 & 579,131 & $7,014,847$ & 1,308 \\
\hline 578,246 & $7,014,501$ & 1,171 & 578,125 & $7,015,431$ & 1,254 & 578,276 & $7,016,635$ & 1,527 & 579,109 & $7,014,827$ & 1,304 \\
\hline 578,254 & $7,014,508$ & 1,172 & 578,107 & $7,015,430$ & 1,258 & 578,267 & $7,016,653$ & 1,526 & 579,064 & 7,014,793 & 1,295 \\
\hline 578,255 & $7,014,527$ & 1,171 & 578,089 & $7,015,428$ & 1,254 & 578,261 & $7,016,667$ & 1,529 & 579,031 & $7,014,776$ & 1,292 \\
\hline 578,248 & $7,014,531$ & 1,171 & 578,083 & $7,015,433$ & 1,256 & 579,918 & $7,016,212$ & 1,466 & 578,995 & $7,014,758$ & 1,290 \\
\hline 578,231 & $7,014,517$ & 1,195 & 578,078 & $7,015,434$ & 1,254 & 579,914 & $7,016,191$ & 1,463 & 578,945 & $7,014,733$ & 1,285 \\
\hline 578,225 & $7,014,525$ & 1,182 & 578,086 & $7,015,446$ & 1,262 & 579,915 & $7,016,173$ & 1,461 & 578,913 & $7,014,715$ & 1,280 \\
\hline 578,214 & $7,014,512$ & 1,181 & 578,086 & $7,015,471$ & 1,270 & 579,919 & $7,016,125$ & 1,455 & 578,882 & 7,014,694 & 1,277 \\
\hline 578,185 & $7,014,520$ & 1,182 & 578,125 & $7,015,507$ & 1,278 & 579,921 & $7,016,101$ & 1,452 & 578,837 & $7,014,691$ & 1,271 \\
\hline 578,165 & $7,014,516$ & 1,181 & 578,149 & $7,015,535$ & 1,283 & 579,897 & $7,016,079$ & 1,450 & 578,790 & $7,014,677$ & 1,268 \\
\hline 578,146 & $7,014,520$ & 1,179 & 578,177 & $7,015,549$ & 1,284 & 579,881 & $7,016,062$ & 1,446 & 578,751 & $7,014,626$ & 1,263 \\
\hline 578,128 & $7,014,522$ & 1,178 & 578,198 & $7,015,560$ & 1,284 & 579,869 & $7,016,034$ & 1,446 & 578,733 & $7,014,607$ & 1,262 \\
\hline 578,111 & $7,014,542$ & 1,179 & 578,211 & $7,015,567$ & 1,284 & 579,855 & $7,016,004$ & 1,444 & 578,707 & $7,014,583$ & 1,262 \\
\hline 578,111 & $7,014,557$ & 1,180 & 578,219 & $7,015,586$ & 1,292 & 579,844 & $7,015,983$ & 1,442 & 578,685 & $7,014,565$ & 1,258 \\
\hline 578,133 & $7,014,566$ & 1,182 & 578,248 & $7,015,621$ & 1,297 & 579,833 & $7,015,959$ & 1,441 & 578,653 & $7,014,558$ & 1,244 \\
\hline 578,143 & $7,014,578$ & 1,185 & 578,277 & $7,015,651$ & 1,308 & 579,819 & $7,015,924$ & 1,435 & 578,632 & $7,014,549$ & 1,240 \\
\hline 578,161 & $7,014,573$ & 1,186 & 578,288 & $7,015,661$ & 1,312 & 579,810 & $7,015,898$ & 1,432 & 578,608 & $7,014,538$ & 1,237 \\
\hline 578,177 & 7,014,605 & 1,188 & 578,320 & $7,015,690$ & 1,322 & 579,797 & $7,015,842$ & 1,424 & 578,588 & $7,014,527$ & 1,233 \\
\hline 578,189 & $7,014,619$ & 1,192 & 578,343 & $7,015,712$ & 1,330 & 579,795 & $7,015,834$ & 1,421 & 578,573 & $7,014,518$ & 1,230 \\
\hline 578,208 & $7,014,635$ & 1,197 & 578,357 & $7,015,728$ & 1,335 & 579,765 & $7,015,788$ & 1,417 & 578,547 & $7,014,489$ & 1,215 \\
\hline 578,235 & $7,014,636$ & 1,199 & 578,357 & $7,015,728$ & 1,334 & 579,759 & $7,015,774$ & 1,413 & 578,528 & $7,014,486$ & 1,209 \\
\hline 578,251 & $7,014,644$ & 1,198 & 578,356 & $7,015,728$ & 1,334 & 579,757 & $7,015,766$ & 1,413 & 578,480 & $7,014,488$ & 1,209 \\
\hline 578,242 & 7,014,652 & 1,197 & 578,367 & $7,015,760$ & 1,342 & 579,744 & $7,015,760$ & 1,404 & 578,461 & $7,014,492$ & 1,194 \\
\hline 578,227 & $7,014,700$ & 1,203 & 578,389 & $7,015,775$ & 1,349 & 579,730 & $7,015,751$ & 1,405 & 578,425 & $7,014,491$ & 1,191 \\
\hline 578,185 & 7,014,691 & 1,202 & 578,399 & $7,015,794$ & 1,357 & 579,724 & $7,015,743$ & 1,405 & 578,402 & 7,014,485 & 1,187 \\
\hline 578,103 & $7,014,641$ & 1,198 & 578,400 & $7,015,826$ & 1,362 & 579,715 & $7,015,725$ & 1,405 & 578,394 & $7,014,491$ & 1,188 \\
\hline 578,066 & $7,014,648$ & 1,196 & 578,394 & $7,015,841$ & 1,365 & 579,705 & $7,015,703$ & 1,407 & 578,375 & $7,014,491$ & 1,186 \\
\hline 578,048 & $7,014,658$ & 1,198 & 578,388 & $7,015,850$ & 1,368 & 579,693 & $7,015,678$ & 1,408 & 578,374 & $7,014,502$ & 1,186 \\
\hline 578,036 & $7,014,712$ & 1,203 & 578,390 & $7,015,864$ & 1,369 & 579,683 & 7,015,667 & 1,408 & 578,375 & $7,014,518$ & 1,187 \\
\hline 578,056 & $7,015,075$ & 1,228 & 578,375 & $7,015,876$ & 1,373 & 579,656 & $7,015,641$ & 1,402 & 578,373 & $7,014,521$ & 1,187 \\
\hline 578,103 & $7,015,154$ & 1,232 & 578,357 & $7,015,914$ & 1,380 & 579,624 & $7,015,604$ & 1,405 & 578,356 & $7,014,509$ & 1,185 \\
\hline 578,128 & $7,015,184$ & 1,231 & 578,352 & $7,015,932$ & 1,382 & 579,597 & $7,015,566$ & 1,400 & 578,344 & $7,014,493$ & 1,186 \\
\hline 578,132 & $7,015,193$ & 1,232 & 578,350 & $7,015,947$ & 1,385 & 579,567 & $7,015,528$ & 1,396 & 578,323 & $7,014,489$ & 1,185 \\
\hline 578,139 & $7,015,214$ & 1,229 & 578,344 & $7,015,972$ & 1,391 & 579,534 & $7,015,489$ & 1,393 & 578,317 & $7,014,497$ & 1,184 \\
\hline 578,156 & $7,015,239$ & 1,231 & 578,344 & $7,015,997$ & 1,393 & 579,514 & $7,015,457$ & 1,390 & 578,305 & $7,014,500$ & 1,182 \\
\hline 578,157 & $7,015,261$ & 1,232 & 578,342 & $7,016,028$ & 1,402 & 579,502 & $7,015,434$ & 1,386 & 578,306 & $7,014,511$ & 1,181 \\
\hline 578,155 & $7,015,274$ & 1,232 & 578,342 & $7,016,046$ & 1,406 & 579,462 & $7,015,378$ & 1,378 & 578,292 & $7,014,509$ & 1,179 \\
\hline 578,165 & 7,015,301 & 1,231 & 578,334 & $7,016,074$ & 1,409 & 579,443 & $7,015,347$ & 1,374 & 578,273 & $7,014,509$ & 1,179 \\
\hline 578,165 & $7,015,303$ & 1,232 & 578,339 & $7,016,118$ & 1,418 & 579,432 & $7,015,318$ & 1,369 & 578,241 & $7,014,495$ & 1,180 \\
\hline 578,165 & $7,015,300$ & 1,234 & 578,331 & 7,016,155 & 1,420 & 579,416 & $7,015,296$ & 1,366 & 578,243 & $7,014,503$ & 1,176 \\
\hline 578,146 & $7,015,299$ & 1,235 & 578,336 & $7,016,194$ & 1,432 & 579,395 & $7,015,260$ & 1,360 & 578,251 & $7,014,510$ & 1,175 \\
\hline 578,144 & $7,015,312$ & 1,236 & 578,339 & $7,016,213$ & 1,432 & 579,384 & $7,015,241$ & 1,356 & & Péwé & \\
\hline $\begin{array}{l}578,145 \\
578,157\end{array}$ & $\begin{array}{l}7,015,329 \\
7,015,341\end{array}$ & $\begin{array}{l}1,238 \\
1,241\end{array}$ & $\begin{array}{l}578,340 \\
578336\end{array}$ & $\begin{array}{l}7,016,248 \\
7016336\end{array}$ & $\begin{array}{l}1,434 \\
1.435\end{array}$ & $\begin{array}{l}579,355 \\
579318\end{array}$ & $\begin{array}{l}7,015,211 \\
7015162\end{array}$ & $\begin{array}{l}1,351 \\
1,343\end{array}$ & 576,816 & 7,012,769 & 1,152 \\
\hline $\begin{array}{l}578,157 \\
578,160\end{array}$ & $\begin{array}{l}7,015,341 \\
7,015,366\end{array}$ & $\begin{array}{l}1,241 \\
1,254\end{array}$ & $\begin{array}{l}578,336 \\
578,328\end{array}$ & $\begin{array}{l}7,016,336 \\
7,016,372\end{array}$ & $\begin{array}{l}1,435 \\
1,441\end{array}$ & $\begin{array}{l}579,318 \\
579,284\end{array}$ & $\begin{array}{l}\text {,015,162 } \\
7,015,100\end{array}$ & $\begin{array}{l}1,045 \\
1,333\end{array}$ & 576,816 & $7,012,769$ & 1,151 \\
\hline 578,160 & $7,015,394$ & 1,252 & 578,319 & $7,016,441$ & $\begin{array}{l}1,4+1 \\
1,444\end{array}$ & 579,260 & $7,015,066$ & $\begin{array}{l}1,035 \\
1,328\end{array}$ & 576,817 & $7,012,770$ & 1,151 \\
\hline 578,154 & $7,015,413$ & 1,248 & 578,316 & $7,016,477$ & 1,494 & 579,236 & $7,015,013$ & 1,321 & & & \\
\hline
\end{tabular}


Table 7. Global Positioning System terminus-position surveys, Gulkana Glacier, September 16, 1999.

[Universal Transverse Mercator (UTM) coordinates are in UTM zone 6. Easting and northing measurements are in meters. NAD 83, North American Datum of 1983; NGVD 29, National Geodetic Vertical Datum of 1929]

\begin{tabular}{|c|c|c|c|c|c|c|c|c|c|c|c|}
\hline Easting & Northing & Altitude & Easting & Northing & Altitude & Easting & Northing & Altitude & Easting & Northing & Altitude \\
\hline \multicolumn{2}{|c|}{ (NAD 83) } & (NGVD 29) & \multicolumn{2}{|c|}{ (NAD 83) } & (NGVD 29) & \multicolumn{2}{|c|}{ (NAD 83) } & (NGVD 29) & \multicolumn{2}{|c|}{ (NAD 83) } & (NGVD 29) \\
\hline \multicolumn{3}{|c|}{ Péwé } & \multicolumn{3}{|c|}{ Terminus positions } & \multicolumn{3}{|c|}{ Terminus positions } & \multicolumn{3}{|c|}{ Terminus positions } \\
\hline 576,813 & $7,012,768$ & 1,155 & 578,144 & $7,015,247$ & 1,222 & 578,343 & $7,016,328$ & 1,461 & 579,226 & $7,014,958$ & 1,317 \\
\hline 576,813 & $7,012,769$ & 1,155 & 578,148 & $7,015,267$ & 1,223 & 578,335 & $7,016,412$ & 1,482 & 579,208 & $7,014,930$ & 1,313 \\
\hline \multirow{2}{*}{\multicolumn{3}{|c|}{ Terminus positions }} & 578,151 & $7,015,279$ & 1,225 & 578,327 & $7,016,456$ & 1,494 & 579,186 & $7,014,901$ & 1,309 \\
\hline & & & 578,159 & $7,015,296$ & 1,226 & 578,306 & $7,016,507$ & 1,507 & 579,156 & $7,014,877$ & 1,305 \\
\hline 578,261 & $7,014,519$ & 1,164 & 578,160 & $7,015,308$ & 1,226 & 578,295 & $7,016,568$ & 1,520 & 579,102 & $7,014,829$ & 1,298 \\
\hline 578,260 & $7,014,535$ & 1,169 & 578,151 & $7,015,321$ & 1,229 & 578,279 & $7,016,628$ & 1,537 & 579,066 & $7,014,801$ & 1,292 \\
\hline 578,256 & $7,014,538$ & 1,166 & 578,168 & $7,015,350$ & 1,239 & 578,271 & $7,016,659$ & 1,546 & 579,012 & $7,014,771$ & 1,285 \\
\hline 578,231 & $7,014,542$ & 1,166 & 578,178 & $7,015,369$ & 1,241 & 579,936 & $7,016,379$ & 1,484 & 578,978 & $7,014,758$ & 1,285 \\
\hline 578,213 & $7,014,539$ & 1,164 & 578,195 & $7,015,384$ & 1,244 & 579,925 & $7,016,339$ & 1,481 & 578,929 & $7,014,734$ & 1,281 \\
\hline 578,195 & $7,014,537$ & 1,159 & 578,201 & $7,015,407$ & 1,245 & 579,924 & $7,016,327$ & 1,480 & 578,890 & $7,014,708$ & 1,275 \\
\hline 578,173 & $7,014,528$ & 1,158 & 578,209 & $7,015,435$ & 1,245 & 579,931 & $7,016,296$ & 1,480 & 578,837 & $7,014,698$ & 1,267 \\
\hline 578,153 & $7,014,526$ & 1,160 & 578,214 & $7,015,452$ & 1,244 & 579,928 & $7,016,275$ & 1,477 & 578,810 & $7,014,701$ & 1,263 \\
\hline 578,150 & $7,014,532$ & 1,160 & 578,221 & $7,015,485$ & 1,245 & 579,918 & $7,016,238$ & 1,472 & 578,782 & $7,014,669$ & 1,263 \\
\hline 578,134 & $7,014,531$ & 1,157 & 578,192 & $7,015,469$ & 1,243 & 579,922 & $7,016,204$ & 1,470 & 578,745 & $7,014,631$ & 1,258 \\
\hline 578,143 & $7,014,544$ & 1,160 & 578,176 & $7,015,454$ & 1,242 & 579,921 & $7,016,166$ & 1,464 & 578,726 & $7,014,611$ & 1,255 \\
\hline 578,164 & $7,014,566$ & 1,161 & 578,159 & $7,015,424$ & 1,236 & 579,930 & $7,016,134$ & 1,461 & 578,701 & $7,014,581$ & 1,250 \\
\hline 578,189 & $7,014,619$ & 1,168 & 578,143 & $7,015,414$ & 1,236 & 579,930 & $7,016,127$ & 1,463 & 578,680 & $7,014,566$ & 1,242 \\
\hline 578,217 & $7,014,634$ & 1,174 & 578,136 & $7,015,414$ & 1,236 & 579,889 & $7,016,058$ & 1,446 & 578,658 & $7,014,557$ & 1,238 \\
\hline 578,235 & $7,014,634$ & 1,179 & 578,128 & $7,015,429$ & 1,241 & 579,870 & $7,016,040$ & 1,447 & 578,625 & $7,014,543$ & 1,233 \\
\hline 578,234 & $7,014,638$ & 1,179 & 578,106 & $7,015,452$ & 1,252 & 579,849 & $7,015,994$ & 1,443 & 578,588 & $7,014,521$ & 1,225 \\
\hline 578,245 & $7,014,648$ & 1,181 & 578,138 & $7,015,520$ & 1,264 & 579,826 & $7,015,941$ & 1,438 & 578,572 & $7,014,510$ & 1,221 \\
\hline 578,244 & $7,014,673$ & 1,185 & 578,151 & $7,015,531$ & 1,268 & 579,801 & $7,015,880$ & 1,438 & 578,539 & $7,014,489$ & 1,224 \\
\hline 578,212 & $7,014,704$ & 1,187 & 578,178 & $7,015,550$ & 1,270 & 579,787 & $7,015,836$ & 1,436 & 578,522 & $7,014,490$ & 1,229 \\
\hline 578,193 & $7,014,708$ & 1,189 & 578,221 & $7,015,584$ & 1,277 & 579,765 & $7,015,808$ & 1,436 & 578,499 & $7,014,493$ & 1,229 \\
\hline 578,157 & $7,014,688$ & 1,188 & 578,246 & $7,015,616$ & 1,284 & 579,769 & 7,015,789 & 1,416 & 578,484 & 7,014,499 & 1,226 \\
\hline 578,131 & $7,014,695$ & 1,191 & 578,256 & $7,015,622$ & 1,287 & 579,766 & $7,015,770$ & 1,411 & 578,459 & $7,014,496$ & 1,225 \\
\hline 578,118 & 7,014,721 & 1,194 & 578,273 & $7,015,642$ & 1,291 & 579,759 & $7,015,769$ & 1,410 & 578,417 & $7,014,489$ & 1,225 \\
\hline 578,112 & $7,014,735$ & 1,196 & 578,295 & $7,015,661$ & 1,300 & 579,746 & $7,015,764$ & 1,409 & 578,414 & $7,014,494$ & 1,224 \\
\hline 578,184 & $7,014,787$ & 1,205 & 578,321 & $7,015,686$ & 1,308 & 579,729 & $7,015,745$ & 1,409 & 578,394 & $7,014,497$ & 1,224 \\
\hline 578,209 & $7,014,815$ & 1,211 & 578,346 & $7,015,711$ & 1,318 & 579,705 & 7,015,699 & 1,408 & 578,402 & $7,014,520$ & 1,163 \\
\hline 578,230 & $7,014,825$ & 1,210 & 578,362 & $7,015,735$ & 1,322 & 579,692 & $7,015,677$ & 1,408 & 578,414 & $7,014,529$ & 1,163 \\
\hline 578,248 & $7,014,849$ & 1,213 & 578,367 & $7,015,751$ & 1,326 & 579,645 & $7,015,621$ & 1,403 & 578,406 & $7,014,540$ & 1,164 \\
\hline 578,252 & $7,014,858$ & 1,216 & 578,374 & $7,015,765$ & 1,329 & 579,624 & $7,015,590$ & 1,400 & 578,388 & $7,014,533$ & 1,160 \\
\hline 578,256 & $7,014,877$ & 1,217 & 578,392 & $7,015,770$ & 1,334 & 579,588 & $7,015,543$ & 1,394 & 578,362 & $7,014,521$ & 1,158 \\
\hline 578,260 & $7,014,891$ & 1,219 & 578,403 & $7,015,788$ & 1,340 & 579,545 & $7,015,494$ & 1,389 & 578,345 & $7,014,521$ & 1,154 \\
\hline 578,268 & $7,014,920$ & 1,226 & 578,409 & $7,015,812$ & 1,345 & 579,511 & $7,015,436$ & 1,383 & 578,341 & $7,014,524$ & 1,156 \\
\hline 578,279 & 7,014,934 & 1,228 & 578,397 & $7,015,846$ & 1,349 & 579,449 & $7,015,337$ & 1,366 & 578,354 & $7,014,540$ & 1,157 \\
\hline 578,266 & $7,014,938$ & 1,224 & 578,386 & $7,015,882$ & 1,357 & 579,418 & $7,015,283$ & 1,357 & 578,323 & $7,014,529$ & 1,155 \\
\hline 578,238 & $7,014,924$ & 1,222 & 578,366 & $7,015,900$ & 1,358 & 579,390 & $7,015,241$ & 1,351 & 578,315 & $7,014,526$ & 1,154 \\
\hline 578,207 & $7,014,942$ & 1,224 & 578,353 & $7,015,942$ & 1,365 & 579,376 & $7,015,224$ & 1,350 & 578,298 & $7,014,532$ & 1,151 \\
\hline 578,186 & $7,014,967$ & 1,227 & 578,343 & $7,015,969$ & 1,372 & 579,353 & $7,015,206$ & 1,346 & 578,283 & $7,014,530$ & 1,150 \\
\hline 578,144 & $7,014,950$ & 1,225 & 578,344 & $7,016,007$ & 1,373 & 579,332 & $7,015,183$ & 1,341 & 578,273 & $7,014,531$ & 1,151 \\
\hline 578,123 & $7,014,956$ & 1,226 & 578,336 & $7,016,044$ & 1,374 & 579,309 & $7,015,142$ & 1,334 & & Péwé & \\
\hline 578,052 & $7,015,078$ & 1,223 & 578,463 & $7,016,019$ & 1,381 & 579,286 & $7,015,105$ & 1,327 & & & \\
\hline 578,079 & $7,015,126$ & 1,220 & 578,337 & $7,016,077$ & 1,409 & 579,267 & $7,015,076$ & 1,323 & 576,817 & 7,012,773 & 1,151 \\
\hline 578,114 & $7,015,163$ & 1,225 & 578,337 & $7,016,097$ & 1,413 & 579,266 & $7,015,077$ & 1,324 & 576,818 & $7,012,774$ & 1,151 \\
\hline 578,128 & $7,015,192$ & 1,225 & 578,335 & $7,016,185$ & 1,429 & 579,227 & $7,014,992$ & 1,323 & 576,818 & $7,012,774$ & 1,153 \\
\hline 578,131 & $7,015,218$ & 1,221 & 578,342 & $7,016,249$ & 1,444 & 579,234 & $7,014,979$ & 1,320 & & & \\
\hline
\end{tabular}


Table 8. Global Positioning System terminus-position surveys, Gulkana Glacier, October 2, 2000.

[Universal Transverse Mercator (UTM) coordinates are in UTM zone 6. Easting and northing measurements are in meters. NAD 83, North American Datum of 1983; NGVD 29, National Geodetic Vertical Datum of 1929]

\begin{tabular}{|c|c|c|c|c|c|c|c|c|c|c|c|}
\hline Easting & Northing & Altitude & Easting & Northing & Altitude & Easting & Northing & Altitude & Easting & Northing & Altitude \\
\hline \multicolumn{2}{|c|}{ (NAD 83) } & (NGVD 29) & \multicolumn{2}{|c|}{ (NAD 83) } & (NGVD 29) & \multicolumn{2}{|c|}{ (NAD 83) } & (NGVD 29) & \multicolumn{2}{|c|}{ (NAD 83) } & (NGVD 29) \\
\hline \multicolumn{3}{|c|}{ Péwé } & \multicolumn{3}{|c|}{ Terminus positions } & \multicolumn{3}{|c|}{ Terminus positions } & \multicolumn{3}{|c|}{ Terminus positions } \\
\hline 576,810 & $7,012,771$ & 1,158 & 578,176 & $7,015,311$ & 1,233 & 578,335 & $7,016,068$ & 1,385 & 579,240 & $7,015,020$ & 1,325 \\
\hline 576,811 & $7,012,771$ & 1,158 & 578,176 & $7,015,324$ & 1,232 & 578,341 & $7,016,130$ & 1,391 & 579,229 & $7,014,984$ & 1,321 \\
\hline \multirow{2}{*}{\multicolumn{3}{|c|}{ Terminus positions }} & 578,172 & $7,015,344$ & 1,235 & 578,331 & $7,016,150$ & 1,396 & 579,216 & $7,014,947$ & 1,312 \\
\hline & & & 578,177 & $7,015,373$ & 1,240 & 578,330 & $7,016,169$ & 1,402 & 579,188 & $7,014,895$ & 1,305 \\
\hline 578,274 & $7,014,529$ & 1,174 & 578,169 & $7,015,380$ & 1,240 & 578,336 & $7,016,222$ & 1,414 & 579,164 & $7,014,881$ & 1,302 \\
\hline 578,265 & $7,014,543$ & 1,174 & 578,167 & $7,015,393$ & 1,242 & 578,337 & $7,016,263$ & 1,423 & 579,135 & $7,014,856$ & 1,299 \\
\hline 578,250 & $7,014,551$ & 1,174 & 578,168 & $7,015,402$ & 1,242 & 578,337 & $7,016,291$ & 1,432 & 579,101 & $7,014,827$ & 1,294 \\
\hline 578,240 & $7,014,555$ & 1,175 & 578,186 & $7,015,407$ & 1,246 & 578,338 & $7,016,325$ & 1,442 & 579,063 & $7,014,800$ & 1,287 \\
\hline 578,232 & $7,014,564$ & 1,178 & 578,189 & $7,015,435$ & 1,247 & 578,336 & $7,016,376$ & 1,450 & 579,027 & $7,014,779$ & 1,282 \\
\hline 578,210 & $7,014,557$ & 1,173 & 578,188 & $7,015,442$ & 1,242 & 578,329 & $7,016,427$ & 1,465 & 578,965 & $7,014,754$ & 1,280 \\
\hline 578,200 & $7,014,541$ & 1,170 & 578,173 & $7,015,452$ & 1,239 & 578,323 & $7,016,460$ & 1,468 & 578,927 & $7,014,733$ & 1,275 \\
\hline 578,172 & $7,014,539$ & 1,169 & 578,145 & $7,015,417$ & 1,236 & 578,304 & $7,016,507$ & 1,481 & 578,885 & $7,014,708$ & 1,268 \\
\hline 578,163 & $7,014,538$ & 1,170 & 578,134 & $7,015,426$ & 1,237 & 578,291 & $7,016,557$ & 1,493 & 578,832 & 7,014,696 & 1,262 \\
\hline 578,154 & $7,014,552$ & 1,170 & 578,132 & $7,015,435$ & 1,237 & 578,285 & $7,016,587$ & 1,498 & 578,815 & $7,014,698$ & 1,260 \\
\hline 578,173 & $7,014,573$ & 1,172 & 578,133 & $7,015,453$ & 1,246 & 579,884 & $7,016,057$ & 1,461 & 578,780 & $7,014,652$ & 1,257 \\
\hline 578,169 & $7,014,590$ & 1,175 & 578,110 & $7,015,454$ & 1,246 & 579,863 & $7,016,035$ & 1,460 & 578,769 & $7,014,636$ & 1,254 \\
\hline 578,188 & $7,014,605$ & 1,180 & 578,102 & $7,015,465$ & 1,252 & 579,843 & $7,015,991$ & 1,454 & 578,752 & $7,014,630$ & 1,253 \\
\hline 578,184 & $7,014,613$ & 1,181 & 578,121 & $7,015,491$ & 1,256 & 579,819 & $7,015,939$ & 1,447 & 578,718 & $7,014,609$ & 1,254 \\
\hline 578,189 & $7,014,620$ & 1,181 & 578,142 & $7,015,529$ & 1,265 & 579,805 & $7,015,896$ & 1,442 & 578,712 & $7,014,610$ & 1,248 \\
\hline 578,195 & $7,014,619$ & 1,182 & 578,176 & $7,015,554$ & 1,270 & 579,795 & $7,015,846$ & 1,433 & 578,711 & $7,014,606$ & 1,251 \\
\hline 578,207 & $7,014,629$ & 1,184 & 578,205 & $7,015,565$ & 1,270 & 579,779 & $7,015,816$ & 1,428 & 578,686 & $7,014,576$ & 1,245 \\
\hline 578,217 & $7,014,637$ & 1,186 & 578,221 & $7,015,591$ & 1,276 & 579,763 & $7,015,794$ & 1,426 & 578,665 & $7,014,565$ & 1,241 \\
\hline 578,231 & $7,014,631$ & 1,186 & 578,248 & $7,015,621$ & 1,284 & 579,758 & $7,015,777$ & 1,423 & 578,640 & $7,014,552$ & 1,244 \\
\hline 578,242 & $7,014,642$ & 1,186 & 578,279 & $7,015,649$ & 1,293 & 579,749 & $7,015,762$ & 1,422 & 578,614 & $7,014,545$ & 1,226 \\
\hline 578,259 & $7,014,662$ & 1,189 & 578,297 & $7,015,668$ & 1,299 & 579,723 & $7,015,752$ & 1,421 & 578,588 & $7,014,530$ & 1,221 \\
\hline 578,243 & $7,014,661$ & 1,187 & 578,322 & $7,015,686$ & 1,306 & 579,685 & $7,015,678$ & 1,419 & 578,567 & $7,014,513$ & 1,216 \\
\hline 578,226 & $7,014,662$ & 1,187 & 578,355 & $7,015,718$ & 1,318 & 579,633 & $7,015,611$ & 1,408 & 578,535 & $7,014,496$ & 1,200 \\
\hline 578,202 & $7,014,646$ & 1,185 & 578,365 & $7,015,740$ & 1,321 & 579,614 & $7,015,581$ & 1,406 & 578,506 & $7,014,503$ & 1,193 \\
\hline 578,179 & $7,014,631$ & 1,181 & 578,398 & $7,015,782$ & 1,335 & 579,588 & $7,015,553$ & 1,401 & 578,472 & $7,014,509$ & 1,188 \\
\hline 578,159 & $7,014,618$ & 1,178 & 578,416 & $7,015,818$ & 1,340 & 579,553 & $7,015,518$ & 1,395 & 578,429 & $7,014,504$ & 1,184 \\
\hline 578,138 & $7,014,606$ & 1,177 & 578,412 & $7,015,842$ & 1,346 & 579,544 & $7,015,496$ & 1,397 & 578,425 & $7,014,513$ & 1,182 \\
\hline 578,090 & $7,014,606$ & 1,175 & 578,402 & $7,015,859$ & 1,348 & 579,525 & $7,015,465$ & 1,394 & 578,428 & $7,014,526$ & 1,182 \\
\hline 578,065 & $7,015,105$ & 1,223 & 578,391 & $7,015,886$ & 1,353 & 579,507 & $7,015,431$ & 1,388 & 578,420 & $7,014,535$ & 1,180 \\
\hline 578,081 & $7,015,132$ & 1,223 & 578,385 & $7,015,902$ & 1,356 & 579,491 & $7,015,407$ & 1,386 & 578,379 & $7,014,522$ & 1,180 \\
\hline 578,098 & $7,015,165$ & 1,225 & 578,371 & $7,015,918$ & 1,361 & 579,459 & $7,015,356$ & 1,378 & 578,333 & $7,014,527$ & 1,176 \\
\hline 578,108 & $7,015,187$ & 1,222 & 578,359 & $7,015,938$ & 1,365 & 579,425 & $7,015,298$ & 1,367 & 578,302 & $7,014,534$ & 1,173 \\
\hline 578,128 & $7,015,214$ & 1,223 & 578,355 & $7,015,959$ & 1,369 & 579,407 & $7,015,269$ & 1,363 & 578,276 & $7,014,528$ & 1,172 \\
\hline 578,143 & $7,015,237$ & 1,223 & 578,348 & $7,015,972$ & 1,370 & 579,378 & $7,015,231$ & 1,356 & 578,270 & $7,014,533$ & 1,173 \\
\hline 578,154 & $7,015,258$ & 1,227 & 578,350 & $7,015,986$ & 1,370 & 579,329 & $7,015,178$ & 1,348 & & Péwé & \\
\hline 578,160 & $7,015,278$ & 1,228 & 578,346 & $7,016,004$ & 1,370 & 579,284 & $7,015,103$ & 1,335 & & & \\
\hline 578,170 & 7,015,291 & 1,233 & 578,349 & $7,016,034$ & 1,381 & 579,254 & $7,015,051$ & 1,326 & 576,817 & 7,012,771 & 1,147 \\
\hline
\end{tabular}


Table 9. Global Positioning System terminus-position surveys, Gulkana Glacier, August 23, 2001.

[Universal Transverse Mercator (UTM) coordinates are in UTM zone 6. Easting and northing measurements are in meters. NAD 83, North American Datum of 1983; NGVD 29, National Geodetic Vertical Datum of 1929]

\begin{tabular}{|c|c|c|c|c|c|c|c|c|c|c|c|}
\hline Easting & Northing & Altitude & Easting & Northing & Altitude & Easting & Northing & \multirow{2}{*}{$\frac{\text { Altitude }}{\text { (NGVD 29) }}$} & Easting & Northing & \multirow{2}{*}{$\frac{\text { Altitude }}{\text { (NGVD 29) }}$} \\
\hline \multicolumn{2}{|c|}{ (NAD 83) } & (NGVD 29) & \multicolumn{2}{|c|}{ (NAD 83) } & (NGVD 29) & \multicolumn{2}{|c|}{ (NAD 83) } & & \multicolumn{2}{|c|}{ (NAD 83) } & \\
\hline \multicolumn{3}{|c|}{ Péwé } & \multicolumn{3}{|c|}{ Terminus positions } & \multicolumn{3}{|c|}{ Terminus positions } & \multicolumn{3}{|c|}{ Terminus positions } \\
\hline 576,817 & $7,012,770$ & 1,158 & 578,194 & $7,014,731$ & 1,206 & 578,143 & $7,015,199$ & 1,234 & 578,339 & $7,015,693$ & 1,313 \\
\hline 576,818 & $7,012,770$ & 1,158 & 578,233 & $7,014,766$ & 1,214 & 578,153 & $7,015,212$ & 1,235 & 578,342 & $7,015,700$ & 1,314 \\
\hline 576,818 & $7,012,771$ & 1,158 & 578,234 & $7,014,770$ & 1,215 & 578,152 & $7,015,231$ & 1,235 & 578,350 & $7,015,707$ & 1,321 \\
\hline 576,817 & $7,012,771$ & 1,158 & 578,221 & $7,014,772$ & 1,217 & 578,165 & $7,015,245$ & 1,235 & 578,364 & $7,015,721$ & 1,325 \\
\hline \multirow{2}{*}{\multicolumn{3}{|c|}{ Terminus positions }} & 578,211 & $7,014,775$ & 1,218 & 578,174 & $7,015,254$ & 1,239 & 578,366 & 7,015,737 & 1,325 \\
\hline & & & 578,209 & $7,014,782$ & 1,219 & 578,176 & $7,015,266$ & 1,237 & 578,372 & $7,015,744$ & 1,324 \\
\hline 578,377 & $7,014,543$ & 1,181 & 578,206 & $7,014,786$ & 1,217 & 578,185 & $7,015,276$ & 1,239 & 578,381 & $7,015,750$ & 1,327 \\
\hline 578,369 & $7,014,599$ & 1,181 & 578,216 & $7,014,790$ & 1,221 & 578,195 & $7,015,285$ & 1,242 & 578,386 & $7,015,759$ & 1,331 \\
\hline 578,354 & $7,014,548$ & 1,181 & 578,225 & $7,014,795$ & 1,221 & 578,200 & $7,015,293$ & 1,240 & 578,396 & $7,015,768$ & 1,335 \\
\hline 578,345 & $7,014,539$ & 1,181 & 578,224 & $7,014,803$ & 1,221 & 578,201 & $7,015,305$ & 1,241 & 578,410 & $7,015,792$ & 1,341 \\
\hline 578,329 & $7,014,535$ & 1,184 & 578,232 & $7,014,812$ & 1,223 & 578,189 & $7,015,308$ & 1,239 & 578,413 & $7,015,799$ & 1,342 \\
\hline 578,317 & $7,014,539$ & 1,185 & 578,236 & $7,014,822$ & 1,221 & 578,211 & $7,015,313$ & 1,236 & 578,424 & $7,015,813$ & 1,342 \\
\hline 578,309 & $7,014,537$ & 1,184 & 578,241 & $7,014,826$ & 1,224 & 578,203 & $7,015,317$ & 1,236 & 578,424 & $7,015,813$ & 1,342 \\
\hline 578,291 & $7,014,543$ & 1,183 & 578,254 & $7,014,834$ & 1,223 & 578,215 & $7,015,331$ & 1,245 & 578,422 & $7,015,815$ & 1,345 \\
\hline 578,274 & $7,014,539$ & 1,181 & 578,260 & $7,014,843$ & 1,223 & 578,213 & $7,015,337$ & 1,242 & 578,421 & $7,015,828$ & 1,346 \\
\hline 578,272 & $7,014,541$ & 1,181 & 578,269 & $7,014,859$ & 1,224 & 578,203 & $7,015,340$ & 1,245 & 578,423 & $7,015,841$ & 1,351 \\
\hline 578,269 & $7,014,542$ & 1,180 & 578,281 & $7,014,884$ & 1,224 & 578,187 & $7,015,347$ & 1,245 & 578,419 & $7,015,847$ & 1,351 \\
\hline 578,265 & $7,014,543$ & 1,180 & 578,280 & $7,014,895$ & 1,224 & 578,195 & $7,015,372$ & 1,245 & 578,417 & $7,015,857$ & 1,351 \\
\hline 578,242 & $7,014,556$ & 1,183 & 578,275 & $7,014,913$ & & 578,204 & $7,015,379$ & & 578,411 & & 1,353 \\
\hline 578,232 & $7,014,567$ & 1,185 & 578,289 & $7,014,926$ & 1,224 & 578,213 & 7,015,391 & 1,246 & 578,405 & $7,015,871$ & 1,353 \\
\hline 578,217 & $7,014,565$ & 1,182 & 578,300 & $7,014,949$ & & 578,215 & $7,015,405$ & & 578,404 & & 1,354 \\
\hline 578,206 & $7,014,570$ & 1,184 & 578,281 & $7,014,955$ & 1,223 & 578,217 & $7,015,411$ & 1,250 & 578,397 & $7,015,885$ & 1,358 \\
\hline 578,187 & $7,014,559$ & 1,182 & 578,265 & $7,014,953$ & & 578,225 & $7,015,426$ & & 578,394 & $7,015,892$ & 1,359 \\
\hline 578,182 & $7,014,547$ & 1,180 & 578,243 & $7,014,954$ & 1,223 & 578,220 & $7,015,436$ & 1,251 & 578,390 & $7,015,904$ & 1,362 \\
\hline 578,180 & $7,014,557$ & 1,180 & 578,223 & $7,014,960$ & & 578,226 & $7,015,443$ & & 578,387 & & 1,362 \\
\hline 578,177 & $7,014,563$ & 1,181 & 578,210 & $7,014,970$ & 1,225 & 578,224 & $7,015,450$ & 1,248 & 578,384 & $7,015,919$ & 1,363 \\
\hline 578,175 & $7,014,578$ & 1,185 & 578,208 & $7,014,982$ & & 578,232 & $7,015,456$ & 1,250 & 578,376 & $7,015,932$ & 1,365 \\
\hline 578,168 & $7,014,589$ & 1,184 & 578,208 & 7,014,997 & 1,226 & 578,236 & $7,015,462$ & 1,252 & 578,370 & $7,015,945$ & 1,368 \\
\hline 578,180 & $7,014,598$ & 1,186 & 578,198 & 7,014,997 & 1,225 & 578,245 & $7,015,468$ & 1,252 & 578,372 & $7,015,943$ & 1,368 \\
\hline 578,187 & $7,014,604$ & 1,185 & 578,186 & 7,014,992 & 1,226 & 578,254 & $7,015,489$ & 1,253 & 578,367 & $7,015,958$ & 1,370 \\
\hline 578,187 & $7,014,615$ & 1,188 & 578,175 & 7,014,986 & 1,226 & 578,267 & $7,015,504$ & 1,258 & 578,364 & $7,015,969$ & 1,370 \\
\hline 578,196 & $7,014,623$ & 1,189 & 578,165 & 7,014,982 & 1,225 & 578,274 & $7,015,519$ & 1,260 & 578,358 & $7,015,975$ & 1,371 \\
\hline 578,209 & $7,014,626$ & 1,191 & 578,139 & 7,014,982 & 1,225 & 578,267 & $7,015,524$ & 1,258 & 578,358 & 7,015,991 & 1,376 \\
\hline 578,221 & $7,014,627$ & 1,191 & 578,128 & $7,014,983$ & 1,226 & 578,275 & $7,015,537$ & 1,263 & 578,356 & $7,016,004$ & 1,377 \\
\hline 578,230 & $7,014,630$ & 1,190 & 578,123 & $7,014,978$ & 1,234 & 578,279 & $7,015,544$ & 1,260 & 578,353 & $7,016,014$ & 1,379 \\
\hline 578,239 & $7,014,641$ & 1,192 & 578,132 & $7,015,011$ & 1,246 & 578,281 & $7,015,553$ & 1,260 & 578,352 & $7,016,021$ & 1,380 \\
\hline 578,247 & $7,014,646$ & 1,195 & 578,126 & $7,015,017$ & 1,241 & 578,290 & $7,015,560$ & 1,269 & 578,353 & $7,016,032$ & 1,384 \\
\hline 578,253 & $7,014,649$ & 1,194 & 578,119 & $7,015,024$ & 1,243 & 578,316 & $7,015,580$ & 1,279 & 578,351 & $7,016,042$ & 1,386 \\
\hline 578,264 & $7,014,646$ & 1,195 & 578,112 & $7,015,030$ & 1,241 & 578,330 & $7,015,590$ & 1,283 & 578,345 & $7,016,056$ & 1,388 \\
\hline 578,283 & $7,014,650$ & 1,194 & 578,105 & $7,015,033$ & 1,239 & 578,335 & $7,015,598$ & 1,283 & 578,348 & $7,016,077$ & 1,392 \\
\hline 578,294 & $7,014,666$ & 1,195 & 578,095 & $7,015,037$ & 1,235 & 578,353 & $7,015,617$ & 1,289 & 578,350 & $7,016,098$ & 1,396 \\
\hline 578,293 & $7,014,674$ & 1,194 & 578,078 & $7,015,036$ & 1,241 & 578,355 & $7,015,627$ & 1,292 & 578,342 & $7,016,115$ & 1,399 \\
\hline 578,278 & $7,014,665$ & 1,194 & 578,074 & $7,015,048$ & 1,240 & 578,336 & $7,015,610$ & 1,286 & 578,335 & 7,016,126 & 1,400 \\
\hline 578,266 & $7,014,673$ & 1,194 & 578,076 & $7,015,068$ & 1,239 & 578,305 & $7,015,619$ & 1,298 & 578,279 & $7,015,544$ & 1,260 \\
\hline 578,270 & $7,014,687$ & 1,194 & 578,075 & $7,015,082$ & 1,235 & 578,301 & $7,015,627$ & 1,298 & 578,281 & $7,015,553$ & 1,260 \\
\hline 578,268 & $7,014,696$ & 1,194 & 578,080 & $7,015,099$ & 1,239 & 578,280 & $7,015,630$ & 1,294 & 578,290 & $7,015,560$ & 1,269 \\
\hline 578,259 & $7,014,705$ & 1,194 & 578,093 & $7,015,121$ & 1,238 & 578,297 & $7,015,640$ & 1,294 & 578,316 & $7,015,580$ & 1,279 \\
\hline 578,247 & $7,014,714$ & 1,194 & 578,099 & $7,015,135$ & 1,241 & 578,309 & $7,015,641$ & 1,302 & 578,330 & $7,015,590$ & 1,283 \\
\hline 578,235 & $7,014,718$ & 1,194 & 578,107 & $7,015,149$ & 1,238 & 578,318 & $7,015,652$ & 1,306 & 578,335 & 7,015,598 & 1,283 \\
\hline 578,222 & $7,014,727$ & 1,203 & 578,113 & $7,015,165$ & 1,239 & 578,323 & $7,015,663$ & 1,307 & 578,353 & $7,015,617$ & 1,289 \\
\hline 578,204 & $7,014,731$ & 1,205 & 578,132 & $7,015,182$ & 1,239 & 578,331 & $7,015,679$ & 1,310 & 578,355 & $7,015,627$ & 1,292 \\
\hline
\end{tabular}


Table 9. Global Positioning System terminus-position surveys, Gulkana Glacier, August 23, 2001.—Continued

[Universal Transverse Mercator (UTM) coordinates are in UTM zone 6. Easting and northing measurements are in meters. NAD 83, North American Datum of 1983; NGVD 29, National Geodetic Vertical Datum of 1929]

\begin{tabular}{|c|c|c|c|c|c|c|c|c|c|c|c|}
\hline Easting & Northing & Altitude & Easting & Northing & Altitude & Easting & Northing & \multirow{2}{*}{$\frac{\text { Altitude }}{\text { (NGVD 29) }}$} & Easting & Northing & \multirow{2}{*}{$\frac{\text { Altitude }}{\text { (NGVD 29) }}$} \\
\hline \multicolumn{2}{|c|}{ (NAD 83) } & (NGVD 29) & \multicolumn{2}{|c|}{ (NAD 83) } & (NGVD 29) & \multicolumn{2}{|c|}{ (NAD 83) } & & \multicolumn{2}{|c|}{ (NAD 83) } & \\
\hline \multicolumn{3}{|c|}{ Terminus positions } & \multicolumn{3}{|c|}{ Terminus positions } & \multicolumn{3}{|c|}{ Terminus positions } & \multicolumn{3}{|c|}{ Terminus positions } \\
\hline 578,336 & $7,015,610$ & 1,286 & 579,820 & $7,015,946$ & 1,444 & 579,326 & $7,015,187$ & 1,348 & 578,835 & $7,014,711$ & 1,262 \\
\hline 578,305 & $7,015,619$ & 1,298 & 579,814 & 7,015,936 & 1,442 & 579,318 & $7,015,176$ & 1,352 & 578,815 & $7,014,711$ & 1,262 \\
\hline 578,301 & $7,015,627$ & 1,298 & 579,811 & $7,015,925$ & 1,441 & 579,306 & $7,015,156$ & 1,348 & 578,795 & $7,014,707$ & 1,262 \\
\hline 578,338 & $7,016,324$ & 1,441 & 579,803 & $7,015,905$ & 1,439 & 579,306 & $7,015,155$ & 1,348 & 578,786 & $7,014,697$ & 1,265 \\
\hline 578,338 & $7,016,344$ & 1,445 & 579,796 & $7,015,885$ & 1,439 & 579,299 & $7,015,142$ & 1,347 & 578,783 & $7,014,681$ & 1,266 \\
\hline 578,336 & $7,016,360$ & 1,449 & 579,793 & $7,015,869$ & 1,437 & 579,289 & $7,015,125$ & 1,345 & 578,782 & $7,014,667$ & 1,265 \\
\hline 578,333 & 7,016,377 & 1,453 & 579,792 & $7,015,860$ & 1,436 & 579,277 & $7,015,101$ & 1,340 & 578,786 & $7,014,657$ & 1,266 \\
\hline 578,329 & 7,016,392 & 1,460 & 579,788 & $7,015,850$ & 1,435 & 579,260 & $7,015,083$ & 1,333 & 578,777 & $7,014,656$ & 1,265 \\
\hline 578,331 & $7,016,403$ & 1,459 & 579,786 & $7,015,841$ & 1,433 & 579,252 & $7,015,068$ & 1,332 & 578,763 & $7,014,637$ & 1,258 \\
\hline 578,330 & $7,016,415$ & 1,463 & 579,784 & $7,015,833$ & 1,431 & 579,238 & $7,015,053$ & 1,331 & 578,747 & $7,014,633$ & 1,253 \\
\hline 578,329 & $7,016,425$ & 1,465 & 579,778 & $7,015,821$ & 1,430 & 579,225 & $7,015,043$ & 1,333 & 578,742 & $7,014,627$ & 1,254 \\
\hline 578,328 & $7,016,436$ & 1,467 & 579,772 & $7,015,812$ & 1,430 & 579,214 & $7,015,025$ & 1,325 & 578,726 & $7,014,611$ & 1,252 \\
\hline 578,327 & $7,016,449$ & 1,469 & 579,767 & $7,015,805$ & 1,428 & 579,203 & $7,015,014$ & 1,324 & 578,714 & 7,014,599 & 1,252 \\
\hline 578,322 & $7,016,463$ & 1,474 & 579,762 & $7,015,795$ & 1,426 & 579,192 & $7,014,998$ & 1,325 & 578,693 & $7,014,583$ & 1,247 \\
\hline 578,314 & $7,016,469$ & 1,476 & 579,757 & $7,015,784$ & 1,424 & 579,186 & $7,014,985$ & 1,322 & 578,679 & $7,014,573$ & 1,240 \\
\hline 578,311 & $7,016,486$ & 1,479 & 579,702 & $7,015,703$ & 1,420 & 579,171 & $7,014,964$ & 1,321 & 578,668 & $7,014,567$ & 1,238 \\
\hline 578,306 & 7,016,499 & 1,483 & 579,683 & $7,015,670$ & 1,419 & 579,151 & $7,014,936$ & 1,312 & 578,656 & $7,014,563$ & 1,236 \\
\hline 578,303 & $7,016,515$ & 1,487 & 579,664 & $7,015,646$ & 1,405 & 579,139 & $7,014,920$ & 1,313 & 578,631 & $7,014,555$ & 1,232 \\
\hline 578,300 & $7,016,524$ & 1,489 & 579,640 & $7,015,620$ & 1,401 & 579,122 & $7,014,905$ & 1,313 & 578,614 & $7,014,547$ & 1,226 \\
\hline 578,296 & $7,016,544$ & 1,495 & 579,617 & $7,015,589$ & 1,401 & 579,111 & $7,014,889$ & 1,313 & 578,600 & $7,014,539$ & 1,228 \\
\hline 578,292 & $7,016,559$ & 1,496 & 579,598 & $7,015,560$ & 1,398 & 579,096 & $7,014,875$ & 1,313 & 578,584 & $7,014,525$ & 1,228 \\
\hline 578,291 & $7,016,590$ & 1,506 & 579,576 & $7,015,533$ & 1,395 & 579,087 & $7,014,856$ & 1,312 & 578,562 & $7,014,507$ & 1,228 \\
\hline 578,295 & 7,016,599 & 1,508 & 579,551 & $7,015,507$ & 1,391 & 579,072 & $7,014,838$ & 1,309 & 578,547 & $7,014,486$ & 1,228 \\
\hline 578,291 & 7,016,610 & 1,508 & 579,534 & $7,015,488$ & 1,390 & 579,052 & $7,014,812$ & 1,289 & 578,537 & $7,014,487$ & 1,220 \\
\hline 578,289 & 7,016,626 & 1,513 & 579,514 & $7,015,461$ & 1,391 & 579,049 & $7,014,797$ & 1,289 & 578,521 & $7,014,485$ & 1,226 \\
\hline 579,914 & 7,016,088 & 1,451 & 579,499 & $7,015,440$ & 1,388 & 579,027 & $7,014,788$ & 1,283 & 578,504 & $7,014,485$ & 1,224 \\
\hline 579,903 & $7,016,076$ & 1,450 & 579,490 & $7,015,417$ & 1,385 & 579,008 & $7,014,774$ & 1,282 & 578,505 & $7,014,492$ & 1,225 \\
\hline 579,891 & $7,016,062$ & 1,450 & 579,468 & $7,015,386$ & 1,380 & 578,980 & $7,014,763$ & 1,280 & 578,481 & $7,014,494$ & 1,225 \\
\hline 579,875 & $7,016,051$ & 1,453 & 579,457 & $7,015,367$ & 1,378 & 578,963 & $7,014,759$ & 1,278 & 578,465 & $7,014,496$ & 1,219 \\
\hline 579,860 & $7,016,037$ & 1,453 & 579,446 & $7,015,350$ & 1,375 & 578,943 & $7,014,744$ & 1,272 & 578,447 & $7,014,497$ & 1,222 \\
\hline 579,858 & 7,016,034 & 1,453 & 579,435 & $7,015,324$ & 1,371 & 578,927 & $7,014,737$ & 1,274 & \multicolumn{3}{|c|}{ Péwé } \\
\hline $\begin{array}{l}579,853 \\
579849\end{array}$ & $\begin{array}{l}7,016,024 \\
7016007\end{array}$ & $\begin{array}{l}1,451 \\
1,450\end{array}$ & $\begin{array}{l}579,432 \\
579,421\end{array}$ & $\begin{array}{l}7,015,316 \\
7,015,301\end{array}$ & $\begin{array}{l}1,370 \\
1,366\end{array}$ & $\begin{array}{l}578,908 \\
578,897\end{array}$ & $\begin{array}{l}7,014,724 \\
7,014717\end{array}$ & $\begin{array}{l}1,271 \\
1,270\end{array}$ & 76,814 & $7,012,770$ & 1,152 \\
\hline $\begin{array}{l}5 / 9,849 \\
579,839\end{array}$ & $\begin{array}{l}7,016,007 \\
7,015,993\end{array}$ & $\begin{array}{l}1,450 \\
1,449\end{array}$ & $\begin{array}{l}579,421 \\
579,406\end{array}$ & $\begin{array}{l}7,015,301 \\
7,015,281\end{array}$ & $\begin{array}{l}1,366 \\
1,365\end{array}$ & $\begin{array}{l}578,897 \\
578,887\end{array}$ & 7,014,710 & $\begin{array}{l}1,2 / 0 \\
1,269\end{array}$ & 576,814 & $7,012,770$ & 1,151 \\
\hline 579,836 & $7,015,980$ & 1,448 & 579,391 & $7,015,259$ & 1,361 & 578,876 & $7,014,713$ & 1,268 & 576,813 & $7,012,771$ & 1,151 \\
\hline 579,830 & $7,015,968$ & 1,446 & 579,374 & $7,015,234$ & 1,361 & 578,866 & $7,014,718$ & 1,268 & 576,813 & $7,012,771$ & 1,152 \\
\hline 579,826 & $7,015,957$ & 1,445 & 579,363 & $7,015,220$ & 1,357 & 578,850 & $7,014,710$ & 1,261 & & & \\
\hline 579,823 & 7,015,952 & 1,445 & 579,342 & $7,015,203$ & 1,352 & 578,839 & $7,014,710$ & 1,262 & & & \\
\hline
\end{tabular}

\section{Glacier Surface Altitude}

Change in surface altitude along a profile down the glacier is often the best way to intuitively grasp how a glacier's geometry is changing over time. For example, Mayo and Trabant (1986) used this parameter alone to estimate growth of Gulkana Glacier from 1975 to 1984.
The glacier surface altitude and the uppermost glacier summer surface at each index site were measured during each onsite visit (fig. 6, table 10). We estimated the surface topography of the glacier by establishing a $75-\mathrm{m}$ plane defining local topography around each index site by surveying at least three local surface points. The index-site altitude was taken as the altitude of this plane at the position of the index site (Mayo and Trabant, 1982). Surveyed glacier-surface points typically have an altitude uncertainty of about $0.05 \mathrm{~m}$. 
Site $D$, accumulation zone

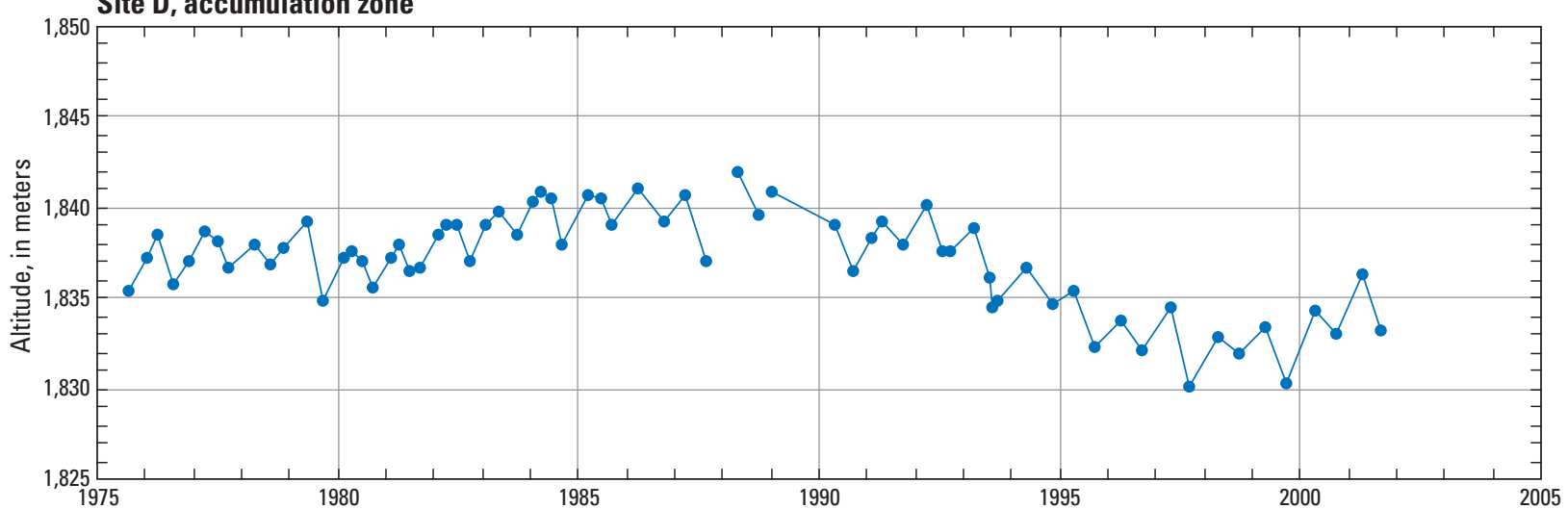

Site B, near equilibrium line

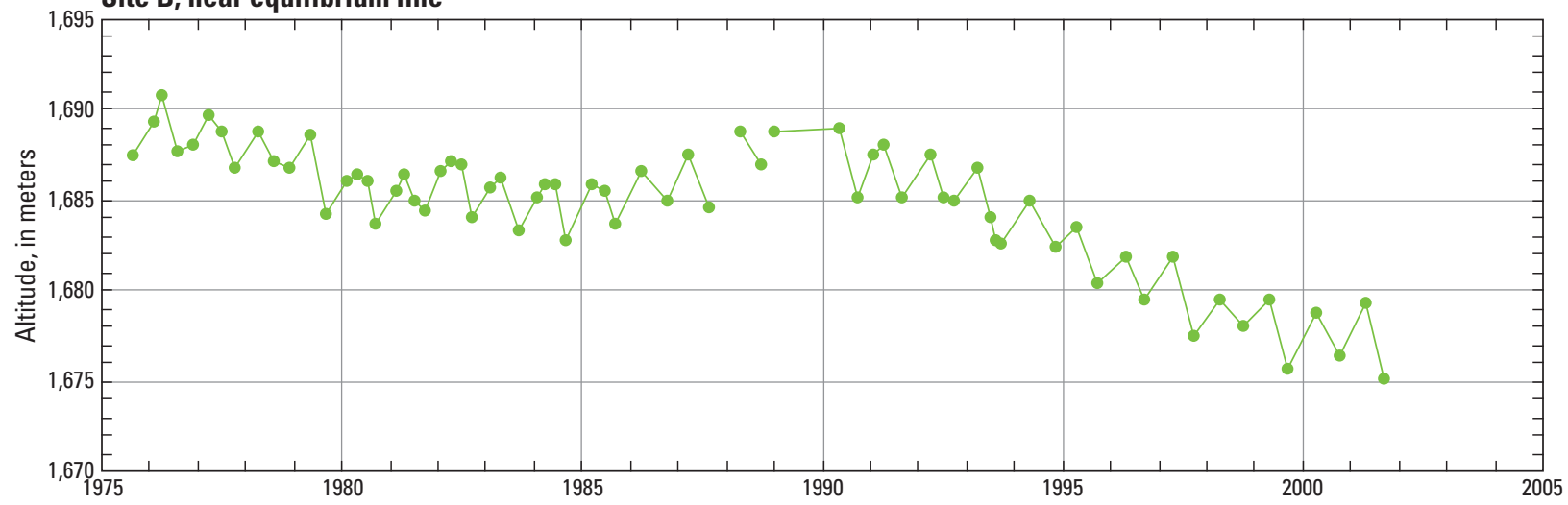

Site $A$, ablation zone

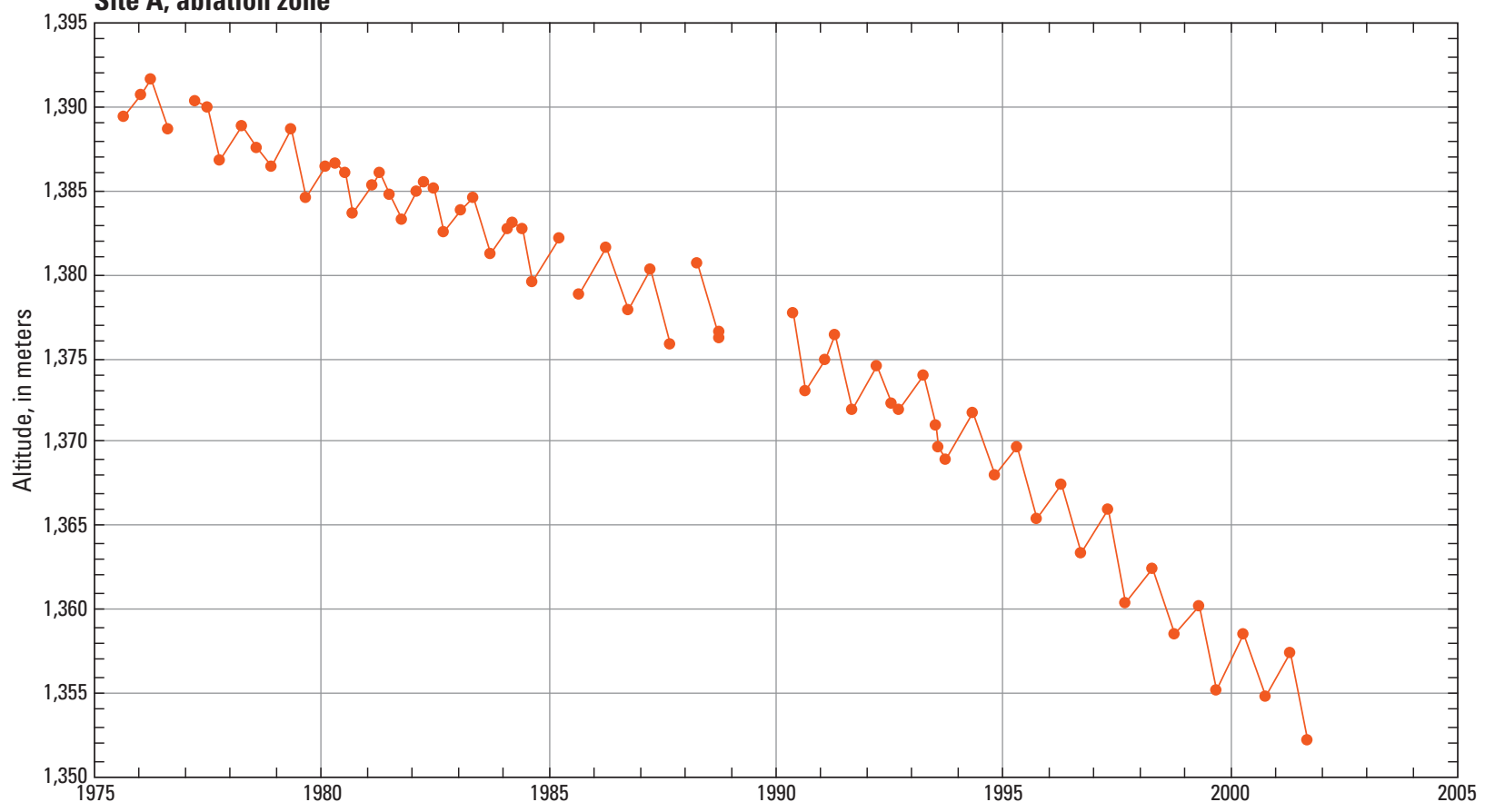

Figure 6. Surface altitudes at index sites A, B, and D, Gulkana Glacier, Alaska. 
Table 10. Gulkana Glacier glacier-surface altitudes at index sites A, B, and D.

[An unresolved datum shift exists between the 1975-87 data and the 1988-96 data. Altitude, in meters; NGVD 29, National Geodetic Vertical Datum of 1929. Abbreviation: mm-dd-yy, month-day-year]

\begin{tabular}{|c|c|c|c|c|c|c|c|}
\hline $\begin{array}{c}\text { Date } \\
\text { (mm-dd-yy) }\end{array}$ & $\begin{array}{c}\text { Site A } \\
\text { (meters) }\end{array}$ & $\begin{array}{c}\text { Site B } \\
\text { (meters) }\end{array}$ & $\begin{array}{c}\text { Site D } \\
\text { (meters) }\end{array}$ & $\begin{array}{c}\text { Date } \\
\text { (mm-dd-yy) }\end{array}$ & $\begin{array}{c}\text { Site A } \\
\text { (meters) }\end{array}$ & $\begin{array}{c}\text { Site B } \\
\text { (meters) }\end{array}$ & $\begin{array}{c}\text { Site D } \\
\text { (meters) }\end{array}$ \\
\hline $08-25-75$ & $1,389.48$ & $1,687.48$ & $1,835.44$ & $10-07-86$ & 1,377.98 & 1,684.95 & 1,839.18 \\
\hline $04-05-76$ & 1,391.73 & $1,690.77$ & $1,838.41$ & $08-28-87$ & $1,375.80$ & $1,684.60$ & $1,837.13$ \\
\hline 08-06-76 & 1,388.59 & $1,687.62$ & 1,835.69 & 04-15-88 & $1,380.67$ & $1,688.85$ & $1,841.93$ \\
\hline $11-22-76$ & & $1,688.10$ & $1,837.01$ & $09-25-88$ & $1,376.29$ & $1,686.91$ & $1,839.66$ \\
\hline $10-03-77$ & 1,386.90 & $1,686.87$ & 1,836.65 & $05-18-90$ & $1,377.70$ & 1,689.04 & 1,839.11 \\
\hline $03-27-78$ & 1,388.90 & $1,688.74$ & $1,838.05$ & $08-17-90$ & $1,373.13$ & $1,685.11$ & $1,836.56$ \\
\hline 08-04-78 & $1,387.60$ & $1,687.19$ & $1,836.90$ & 02-02-91 & $1,374.87$ & $1,687.44$ & 1,838.31 \\
\hline $11-18-78$ & $1,386.46$ & $1,686.79$ & 1,837.78 & 04-18-91 & $1,376.35$ & $1,688.14$ & $1,839.23$ \\
\hline 05-04-79 & 1,388.71 & $1,688.57$ & 1,839.20 & 09-06-91 & $1,371.94$ & $1,685.16$ & $1,837.94$ \\
\hline $09-16-80$ & 1,383.65 & 1,683.69 & 1,835.64 & 07-12-93 & $1,371.07$ & 1,683.97 & $1,836.13$ \\
\hline 02-19-81 & $1,385.40$ & $1,685.49$ & 1,837.18 & 08-03-93 & $1,369.64$ & $1,682.83$ & $1,834.56$ \\
\hline $04-17-81$ & 1,386.16 & $1,686.47$ & 1,837.89 & 09-22-93 & $1,368.97$ & $1,682.53$ & $1,834.79$ \\
\hline $07-10-81$ & 1,384.76 & $1,684.92$ & $1,836.57$ & $04-28-94$ & $1,371.84$ & $1,685.01$ & $1,836.75$ \\
\hline $10-01-81$ & 1,383.33 & $1,684.45$ & 1,836.66 & $10-31-94$ & $1,368.01$ & 1,682.32 & $1,834.66$ \\
\hline 01-27-82 & 1,384.89 & $1,686.55$ & $1,838.45$ & 04-19-95 & 1,369.64 & $1,683.54$ & $1,835.37$ \\
\hline $04-12-82$ & $1,385.56$ & $1,687.08$ & 1,839.14 & 09-26-95 & $1,365.46$ & $1,680.41$ & $1,832.29$ \\
\hline 06-19-82 & 1,385.13 & 1,686.92 & 1,839.13 & 04-18-96 & $1,367.50$ & $1,681.81$ & $1,833.71$ \\
\hline 09-13-82 & 1,382.45 & $1,684.03$ & 1,836.98 & 09-16-96 & 1,363.39 & $1,679.52$ & $1,832.08$ \\
\hline 02-03-83 & 1,383.82 & $1,685.72$ & 1,839.12 & 04-19-97 & 1,365.92 & 1,681.86 & $1,834.44$ \\
\hline 05-04-83 & 1,384.59 & $1,686.25$ & 1,839.80 & 09-15-97 & $1,360.39$ & $1,677.40$ & $1,830.12$ \\
\hline 09-04-85 & 1,378.82 & $1,683.76$ & 1,838.99 & 09-10-01 & $1,352.22$ & $1,675.15$ & $1,833.18$ \\
\hline 03-30-86 & 1,381.66 & $1,686.68$ & 1,841.11 & & & & \\
\hline
\end{tabular}


Additionally, the locations used to define the plane of the glacier surface may not be representative of the average glacier surface; hence, extrapolating along this plane to the index site may introduce further error. The glacier-surface orientation and slope determinations have a small random variability that is used to assess the magnitude of this error. Because this error is site specific, depending largely on the local surface roughness of the glacier, an average glaciersurface slope error of $0.5 \mathrm{grad}$ was applied to the distance between the closest surveyed point and the index site. This error, combined with the surveying error, yielded an average error of $0.15 \mathrm{~m}$ for the index-site altitudes.

\section{Ice Motion}

Surface displacement at index sites was measured by optical surveying of balance stakes. Figure 7 shows 19962001 stake locations and displacements at site B. The stakes were kept within about 1 year's displacement of the index site (usually less than $80 \mathrm{~m}$ ) to maximize the year-to-year comparability of the motion (Mayo and others, 1979; Mayo and Trabant, 1982) and were replaced as necessary. Reported stake locations (tables 11-13) have been corrected for changes in stake geometry including bends, bows and leans (March, 2000). Leaning stakes are most commonly observed at Gulkana Glacier, and when a bend or bow does occur, it is generally at or above the most-recent summer surface. Aside from changes in stake geometry, location uncertainty is largely a result of the survey-control net errors (GPS for horizontal and optical surveying for vertical) and resection errors. Resection was conducted by surveying four or five backsight targets, instead of the minimum of three backsight targets, to allow error evaluation. The net and resection errors combined yield position errors of about $0.15 \mathrm{~m}$ in the horizontal and $0.05 \mathrm{~m}$ in the vertical. The error of extrapolating to the bottom of the stake is estimated to be $0.15 \mathrm{~m}$, resulting in a total horizontal error for stake-bottom positions of $0.2 \mathrm{~m}$. Vertical errors are significantly less, about $0.05 \mathrm{~m}$. Hence, reported displacements have errors of about $0.3 \mathrm{~m}$.

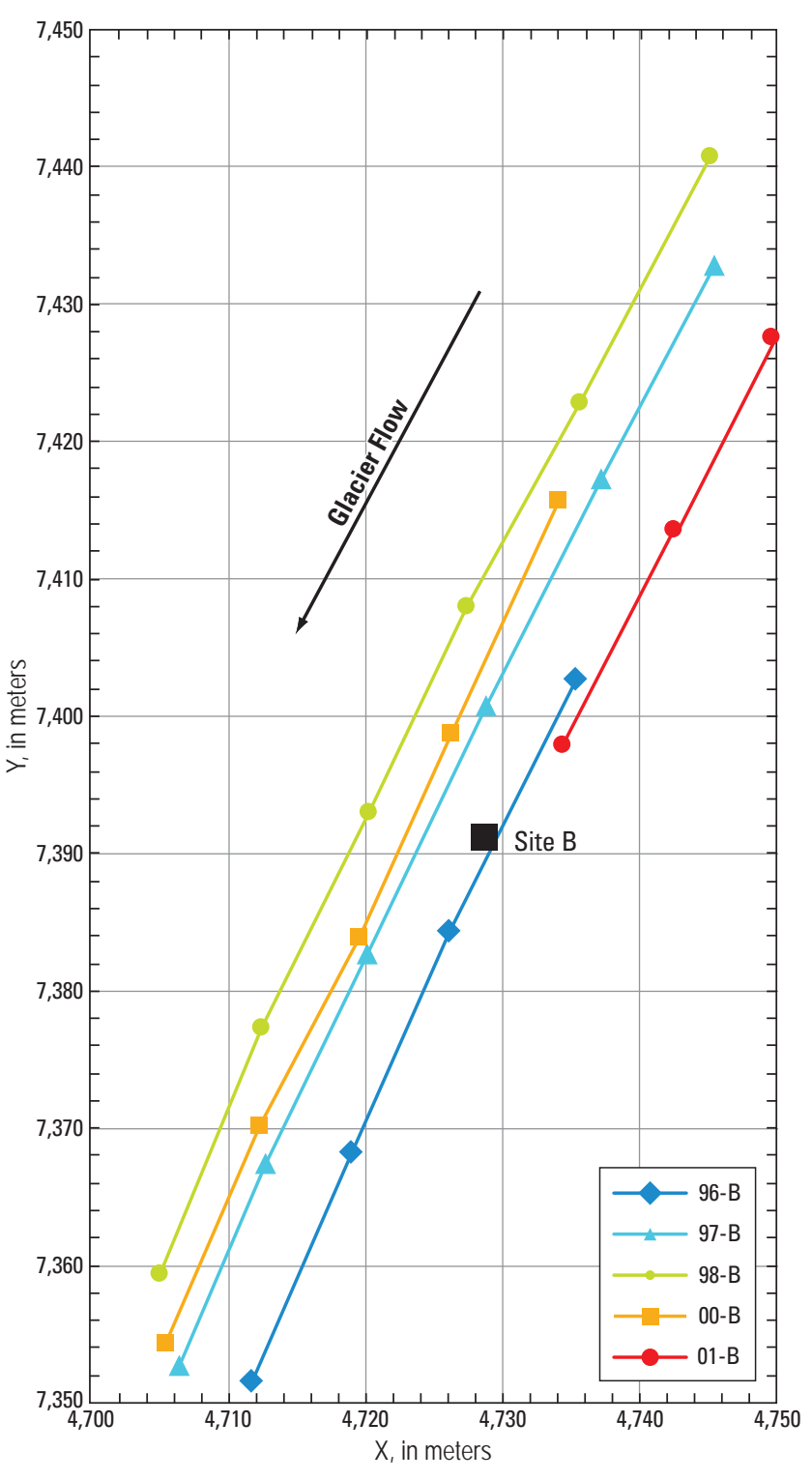

Figure 7. Locations of stakes at site B, Gulkana Glacier, Alaska, shown as an example. First two characters of stake name represent the last two digits of the year the stake was installed. Two locations for stake $96-\mathrm{B}$ are from previous report (March, 2003). 
Table 11. Glacier surface slope, stake bottom locations, and stake motion determined from optical surveys, Site A, 1996-2001.

[Stake name: The first two digits represent the year the stake was installed; letters (A, B, D) represent the index site on the glacier (fig. 2); and a number following the letter is used to differentiate multiple stakes installed at the same site in 1 year. $\theta$ is the downdip direction with zero east and positive counterclockwise. $\varphi$ is the dip angle with zero horizontal and positive angles up. $\mathrm{X}_{\mathrm{S}}, \mathrm{Y}_{\mathrm{S}}, \mathrm{Z}_{\mathrm{S}}$, stake bottom location. $\Delta \mathrm{XYZ}$ is the total three-dimensional displacement of the stake bottom between measurements. Horizontal displacement angle is measured positive counterclockwise; zero is east. Vertical displacement angle is measured positive up from horizontal. Seasonal speed is the stake displacement divided by the measurement period in years. Annual speed is the stake displacement divided by the measurement period in years for two or more measurement periods, so that the period is close to a year. Grad is a unit of measure for a plane angle and is equal to the plane angle in degrees multiplied by (100/90). Abbreviations: mm-dd-yy, month-day-year; m, meter; m/yr, meter per year]

\begin{tabular}{|c|c|c|c|c|c|c|c|c|c|c|c|c|c|}
\hline \multirow{3}{*}{$\begin{array}{c}\text { Date } \\
\text { (mm-dd-yy) }\end{array}$} & \multirow{3}{*}{$\begin{array}{l}\text { Stake } \\
\text { name }\end{array}$} & \multicolumn{3}{|c|}{ Glacier surface } & \multicolumn{3}{|c|}{ Stake bottom } & \multirow{3}{*}{$\begin{array}{l}\text { Period } \\
\text { (days) }\end{array}$} & \multirow{3}{*}{$\begin{array}{c}\Delta X Y Z \\
(\mathrm{~m})\end{array}$} & \multicolumn{2}{|c|}{$\begin{array}{l}\text { Displacement } \\
\text { angles }\end{array}$} & \multirow{3}{*}{$\begin{array}{c}\text { Seasonal } \\
\text { speed } \\
\text { (m/yr) }\end{array}$} & \multirow{3}{*}{$\begin{array}{c}\text { Annual } \\
\text { speed } \\
\text { (m/yr) }\end{array}$} \\
\hline & & $\theta_{g}$ & & $\phi_{g}$ & $\mathrm{X}_{\mathrm{s}}$ & $\mathbf{Y}_{\mathrm{S}}$ & $\mathrm{Z}_{\mathrm{s}}$ & & & Horizontal & Vertical & & \\
\hline & & \multicolumn{3}{|c|}{ (grad) } & \multicolumn{3}{|c|}{ (m) } & & & \multicolumn{2}{|c|}{ (grad) } & & \\
\hline $04-17-96$ & 96-A & -154.95 & - & 7.06 & $3,802.06$ & $4,477.24$ & $1,358.14$ & & & & & & \\
\hline 09-16-96 & 96-A & -144.50 & - & 7.20 & $3,796.46$ & $4,471.07$ & $1,358.40$ & 152 & 8.34 & -146.92 & 1.93 & 20.03 & \\
\hline 04-18-97 & $96-A$ & -149.37 & - & 7.16 & 3,791.09 & $4,465.10$ & $1,358.51$ & 214 & 8.03 & -146.65 & 0.86 & 13.70 & 16.3 \\
\hline $04-17-96$ & 96-A1 & -154.95 & - & 7.06 & $3,810.54$ & $4,470.37$ & $1,358.42$ & & & & & & \\
\hline 09-16-96 & 96-A1 & -144.50 & - & 7.20 & $3,804.79$ & $4,464.43$ & $1,358.66$ & 152 & 8.27 & -148.95 & 1.88 & 19.86 & \\
\hline 04-18-97 & 96-A1 & -149.37 & - & 7.16 & 3,799.60 & $4,458.51$ & $1,358.76$ & 214 & 7.88 & -145.86 & 0.84 & 13.44 & 16.1 \\
\hline 04-18-97 & 97-A & -149.37 & - & 7.16 & $3,805.05$ & $4,465.16$ & $1,355.31$ & & & & & & \\
\hline 09-15-97 & 97-A & -144.67 & - & 6.79 & $3,799.58$ & $4,459.88$ & $1,355.59$ & 150 & 7.60 & -151.17 & 2.33 & 18.49 & \\
\hline $04-17-98$ & $97-A$ & -146.96 & - & 6.68 & 3,794.58 & $4,454.87$ & $1,355.71$ & 214 & 7.08 & -149.89 & 1.09 & 12.08 & 14.7 \\
\hline 04-18-97 & 97-AA & -149.37 & - & 7.16 & $3,832.08$ & $4,466.73$ & $1,357.48$ & & & & & & \\
\hline 09-15-97 & 97-AA & -144.67 & - & 6.79 & $3,826.76$ & $4,460.95$ & $1,357.74$ & 150 & 7.86 & -147.39 & 2.07 & 19.13 & \\
\hline 04-17-98 & 97-AA & -146.96 & - & 6.68 & $3,821.70$ & 4,455.69 & $1,357.84$ & 214 & 7.30 & -148.76 & 0.92 & 12.45 & 15.2 \\
\hline $04-17-98$ & 98-A & -146.96 & - & 6.68 & $3,830.84$ & 4,455.99 & $1,353.46$ & & & & & & \\
\hline 09-23-98 & 98-A & -160.52 & - & 7.20 & $3,825.90$ & $4,450.60$ & 1,353.66 & 159 & 7.31 & -147.21 & 1.79 & 16.78 & \\
\hline 04-22-99 & 98-A & -149.20 & - & 7.99 & $3,821.43$ & $4,445.90$ & $1,353.80$ & 211 & 6.49 & -148.40 & 1.32 & 11.23 & 13.6 \\
\hline 04-17-98 & 98-A2 & -146.96 & - & 6.68 & $3,824.76$ & $4,461.74$ & $1,353.55$ & & & & & & \\
\hline 09-23-98 & 98-A2 & -160.52 & - & 7.20 & $3,819.96$ & $4,456.54$ & $1,353.77$ & 159 & 7.08 & -147.42 & 1.97 & 16.25 & \\
\hline 04-22-99 & 98-A2 & -149.20 & - & 7.99 & $3,815.48$ & $4,451.96$ & $1,353.90$ & 211 & 6.41 & -149.29 & 1.26 & 11.09 & 13.3 \\
\hline 04-22-99 & 99-A & -149.20 & - & 7.99 & 3,839.34 & 4,458.94 & $1,352.45$ & & & & & & \\
\hline 09-14-99 & 99-A & -168.66 & - & 8.80 & $3,835.30$ & $4,454.87$ & $1,352.55$ & 145 & 5.73 & -149.79 & 1.12 & 14.42 & \\
\hline $04-21-00$ & 99-A & -154.01 & - & 7.76 & 3,830.93 & $4,450.37$ & $1,352.81$ & 220 & 6.28 & -149.02 & 2.64 & 10.42 & 12.0 \\
\hline 04-22-99 & 99-A1 & -149.20 & - & 7.99 & $3,832.55$ & $4,465.45$ & $1,352.41$ & & & & & & \\
\hline 09-14-99 & 99-A1 & -168.66 & - & 8.80 & $3,828.80$ & $4,461.15$ & $1,352.56$ & 145 & 5.71 & -145.74 & 1.68 & 14.37 & \\
\hline 04-21-00 & 99-A1 & -154.01 & - & 7.76 & $3,825.29$ & $4,456.63$ & $1,353.00$ & 220 & 5.74 & -142.01 & 4.81 & 9.52 & 11.4 \\
\hline $04-21-00$ & $00-A$ & -154.01 & - & 7.76 & $3,834.76$ & $4,454.07$ & 1,349.10 & & & & & & \\
\hline $10-01-00$ & $00-A$ & -152.47 & - & 7.31 & $3,830.48$ & $4,449.27$ & $1,349.38$ & 163 & 6.44 & -146.38 & 2.75 & 14.42 & \\
\hline 04-20-01 & $00-A$ & -159.65 & - & 6.96 & $3,826.60$ & $4,445.00$ & $1,349.61$ & 201 & 5.77 & -146.97 & 2.50 & 10.48 & 12.2 \\
\hline 09-10-01 & 00-A & -159.63 & - & 6.89 & $3,822.71$ & $4,440.44$ & $1,349.80$ & 143 & 5.99 & -144.91 & 2.04 & 15.29 & \\
\hline $04-21-00$ & 00-A1 & -154.01 & - & 7.76 & $3,827.74$ & $4,461.17$ & 1,348.89 & & & & & & \\
\hline $10-01-00$ & 00-A1 & -152.47 & - & 7.31 & $3,823.00$ & $4,456.73$ & 1349.18 & 163 & 6.50 & -152.08 & 2.83 & 14.56 & \\
\hline 04-20-01 & 00-A1 & -159.65 & - & 6.96 & 3,819.31 & $4,452.98$ & $1,349.45$ & 201 & 5.27 & -149.48 & 3.23 & 9.57 & 11.8 \\
\hline 09-10-01 & 00-A1 & -159.63 & - & 6.89 & $3,815.18$ & $4,447.92$ & $1,349.58$ & 143 & 6.54 & -143.55 & 1.33 & 16.69 & \\
\hline 04-20-01 & $01-A$ & -159.65 & - & 6.96 & 3,835.35 & $4,455.43$ & $1,348.98$ & & & & & & \\
\hline 09-10-01 & $01-\mathrm{A}$ & -159.63 & - & 6.89 & 3,831.31 & $4,451.27$ & $1,349.28$ & 143 & 5.81 & -148.99 & 3.28 & 14.83 & \\
\hline Average & & -153.16 & - & 7.31 & & & & & 6.76 & -147.69 & 2.02 & 14.31 & 13.68 \\
\hline Standard d & leviation & 6.56 & & 0.56 & & & & & 0.93 & 2.35 & 0.96 & 3.20 & 1.82 \\
\hline
\end{tabular}


Table 12. Glacier surface slope, stake bottom locations, and stake motion determined from optical surveys, Site B, 1996-2001.

[Stake name: The first two digits represent the year the stake was installed; letters (A, B, D) represent the index site on the glacier (fig. 2); and a number following the letter is used to differentiate multiple stakes installed at the same site in 1 year. $\theta$ is the downdip direction with zero east and positive counterclockwise. $\varphi$ is the dip angle with zero horizontal and positive angles up. $\mathrm{X}, \mathrm{Y}, \mathrm{Z}$, stake bottom location. $\Delta \mathrm{XYZ}$ is the total three-dimensional displacement of the stake bottom between measurements. Horizontal displacement angle is measured positive counterclockwise; zero is east. Vertical displacement angle is measured positive up from horizontal. Seasonal speed is the stake displacement divided by the measurement period in years. Annual speed is the stake displacement divided by the measurement period in years for two or more measurement periods, so that the period is close to a year. Grad is a unit of measure for a plane angle and is equal to the plane angle in degrees multiplied by (100/90). Abbreviations: mm-dd-yy, month-day-year; m, meter; m/yr, meter per year]

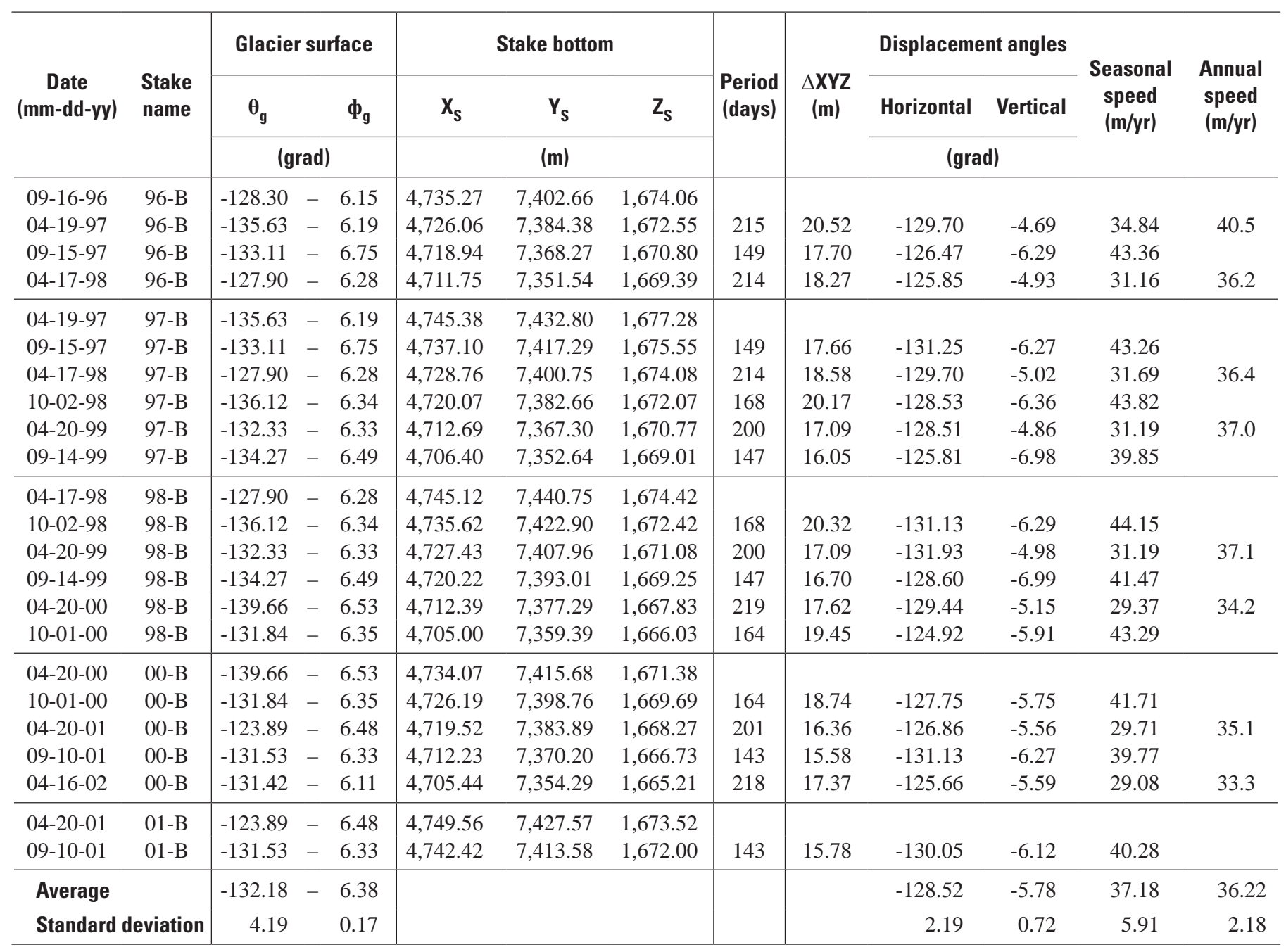


Table 13. Glacier surface slope, stake bottom locations, and stake motion determined from optical surveys, Site D, 1996-2001.

[Stake name: The first two digits represent the year the stake was installed; letters (A, B, D) represent the index site on the glacier (fig. 2); and a number following the letter is used to differentiate multiple stakes installed at the same site in 1 year. $\theta$ is the downdip direction with zero east and positive counterclockwise. $\phi$ is the dip angle with zero horizontal and positive angles up. $\mathrm{X}_{\mathrm{S}}, \mathrm{Y}_{\mathrm{S}}, \mathrm{Z}_{\mathrm{S}}$, stake bottom location. $\Delta \mathrm{XYZ}$ is the total three-dimensional displacement of the stake bottom between measurements. Horizontal displacement angle is measured positive counterclockwise; zero is east. Vertical displacement angle is measured positive up from horizontal. Seasonal speed is the stake displacement divided by the measurement period in years. Annual speed is the stake displacement divided by the measurement period in years for two or more measurement periods, so that the period is close to a year. Grad is a unit of measure for a plane angle and is equal to the plane angle in degrees multiplied by (100/90). Abbreviations: mm-dd-yy, month-day-year m, meter; m/yr, meter per year]

\begin{tabular}{|c|c|c|c|c|c|c|c|c|c|c|c|c|c|}
\hline \multirow{3}{*}{$\begin{array}{c}\text { Date } \\
\text { (mm-dd-yy) }\end{array}$} & \multirow{3}{*}{$\begin{array}{l}\text { Stake } \\
\text { name }\end{array}$} & \multicolumn{3}{|c|}{ Glacier surface } & \multicolumn{3}{|c|}{ Stake bottom } & \multirow{3}{*}{$\begin{array}{l}\text { Period } \\
\text { (days) }\end{array}$} & \multirow{3}{*}{$\begin{array}{c}\Delta X Y Z \\
(\mathrm{~m})\end{array}$} & \multicolumn{2}{|c|}{$\begin{array}{c}\text { Displacement } \\
\text { angles }\end{array}$} & \multirow{3}{*}{$\begin{array}{c}\text { Seasonal } \\
\text { speed } \\
(\mathrm{m} / \mathrm{yr})\end{array}$} & \multirow{3}{*}{$\begin{array}{c}\text { Annual } \\
\text { speed } \\
(\mathrm{m} / \mathrm{yr})\end{array}$} \\
\hline & & $\theta_{g}$ & & $\phi_{g}$ & $x_{s}$ & $\mathbf{Y}_{\mathrm{S}}$ & $\mathrm{Z}_{\mathrm{s}}$ & & & Horizontal & Vertical & & \\
\hline & & \multicolumn{3}{|c|}{ (grad) } & \multicolumn{3}{|c|}{ (m) } & & & \multicolumn{2}{|c|}{ (grad) } & & \\
\hline 04-18-96 & 94-D & 182.80 & - & 6.22 & $5,959.1$ & 7,379.8 & $1,819.4$ & & & & & & \\
\hline 09-16-96 & 94-D & -196.20 & - & 6.93 & $5,940.7$ & 7,390.6 & $1,817.0$ & 151 & 21.5 & 166.4 & -7.1 & 51.9 & \\
\hline 04-18-96 & 96-D & 182.80 & - & 6.22 & $6,020.5$ & 7,339.7 & $1,826.8$ & & & & & & \\
\hline 09-16-96 & 96-D & -196.20 & - & 6.93 & $6,002.4$ & 7,350.6 & $1,824.2$ & 151 & 21.2 & 165.5 & -7.7 & 51.3 & \\
\hline 04-19-97 & 96-D & -197.11 & - & 8.10 & $5,978.2$ & 7,364.3 & $1,821.2$ & 215 & 28.0 & 167.3 & -6.7 & 47.5 & 49.1 \\
\hline $09-15-97$ & 96-D & 183.30 & - & 6.85 & 5,962.2 & 7,374.1 & 1,819.2 & 149 & 18.9 & 164.8 & -7.0 & 46.2 & \\
\hline $04-15-98$ & 96-D & 196.96 & - & 8.10 & $5,944.4$ & 7,384.9 & 1,817.1 & 212 & 20.9 & 165.5 & -6.3 & 36.1 & 40.2 \\
\hline 04-20-99 & 97-D & 167.38 & - & 7.00 & $5,954.5$ & 7,378.1 & 1,818.3 & 200 & 20.4 & 164.6 & -6.7 & 37.2 & 40.7 \\
\hline 09-14-99 & 97-D & 171.97 & - & 7.13 & 5,939.6 & $7,387.0$ & $1,816.4$ & 147 & 17.4 & 165.8 & -6.7 & 43.2 & \\
\hline $04-15-98$ & 98-D & 196.96 & - & 8.10 & $6,031.1$ & 7,329.9 & $1,827.2$ & & & & & & \\
\hline $10-02-98$ & 98-D & 196.72 & - & 8.06 & 6,013.3 & 7,340.6 & $1,824.4$ & 170 & 21.0 & 165.5 & -8.7 & 45.1 & \\
\hline 04-20-99 & 98-D & 167.38 & - & 7.00 & $5,996.1$ & 7,351.2 & 1,822.1 & 200 & 20.3 & 164.8 & -6.9 & 37.1 & 40.8 \\
\hline 09-14-99 & 98-D & 171.97 & - & 7.13 & $5,981.2$ & 7,360.2 & $1,820.2$ & 147 & 17.5 & 165.5 & -7.0 & 43.4 & \\
\hline $04-20-00$ & 98-D & 181.74 & - & 7.48 & $5,962.3$ & 7,371.3 & 1,818.0 & 219 & 22.1 & 166.0 & -6.4 & 36.8 & 39.4 \\
\hline $04-20-00$ & 00-D & 181.74 & - & 7.48 & 6,019.9 & 7,333.9 & $1,827.2$ & & & & & & \\
\hline $10-01-00$ & 00-D & 177.11 & - & 7.92 & $6,002.9$ & 7,344.9 & 1,825.1 & 164 & 20.3 & 163.7 & -6.9 & 45.2 & \\
\hline 04-19-01 & 00-D & 182.01 & - & 6.74 & $5,984.4$ & 7,355.9 & 1,823.1 & 200 & 21.7 & 165.6 & -5.7 & 39.5 & 42.1 \\
\hline
\end{tabular}

\section{Meteorology}

Since October 1967, a weather station has operated at 1,480 m on the eastern ice-cored moraine of Gulkana Glacier (figs. 2 and 3 ; Kennedy and others, 1997) to measure air temperature and precipitation, a location representative of the glacier area meteorology. Since October 1996, wind speed has also been measured.

A radiation-shielded digital temperature sensor with an accuracy of $0.1{ }^{\circ} \mathrm{C}$ and time constant of 10 seconds in air was installed in 1996. The sensor brand is the same as the logger, so we assume the sensor specification is not invalidated by the analog to digital conversion in the logger. The radiation shield is open on the bottom and there is likely some temperature error due to upward radiation when snow is on the ground and/or there is little wind. This is a known problem with virtually all passive radiation shields. This air temperature sensor was sampled every minute and the 15-minute averages were logged and used to determine the daily averages shown in figures 8-12 and listed in tables 14-18. Following the National Climatic Data Center (1996) convention, monthly mean temperatures were calculated for months with nine or fewer missing daily values. 


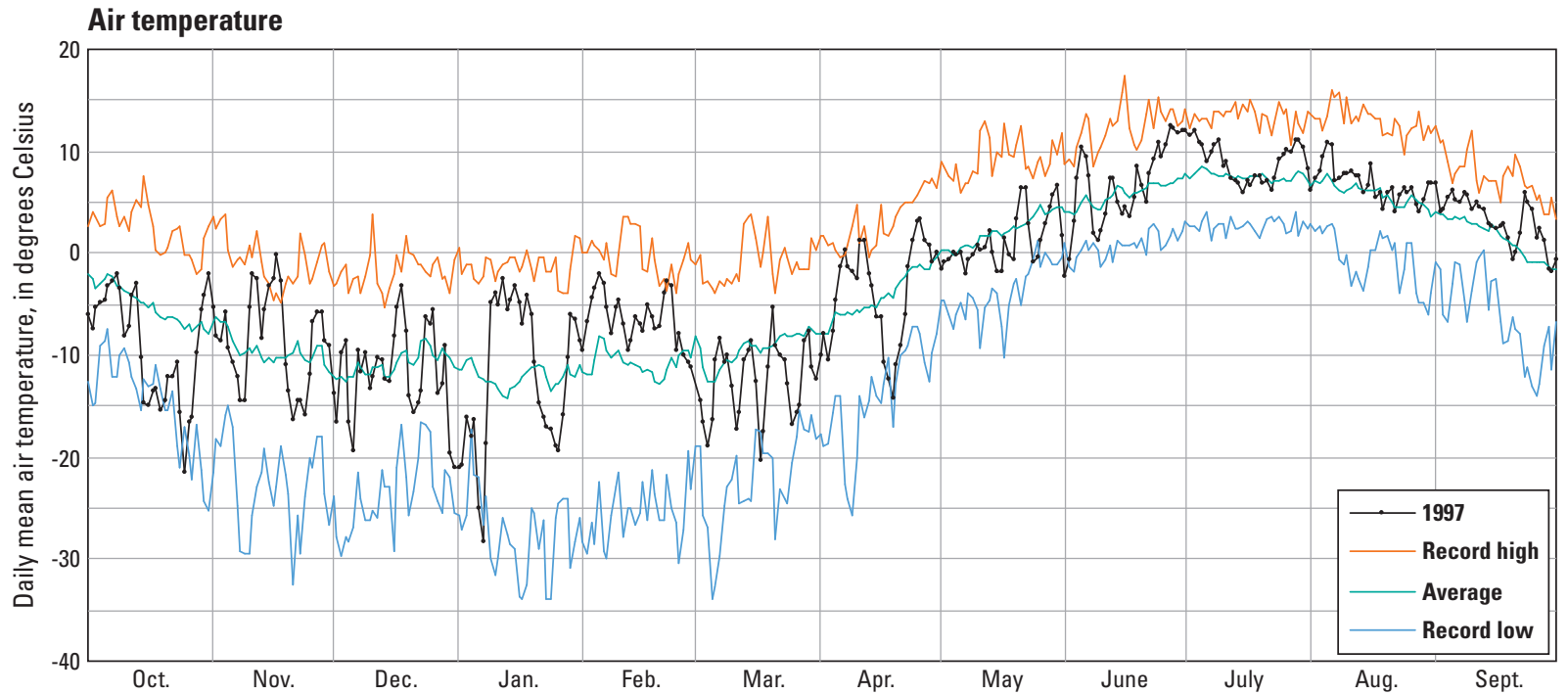

Precipitation catch
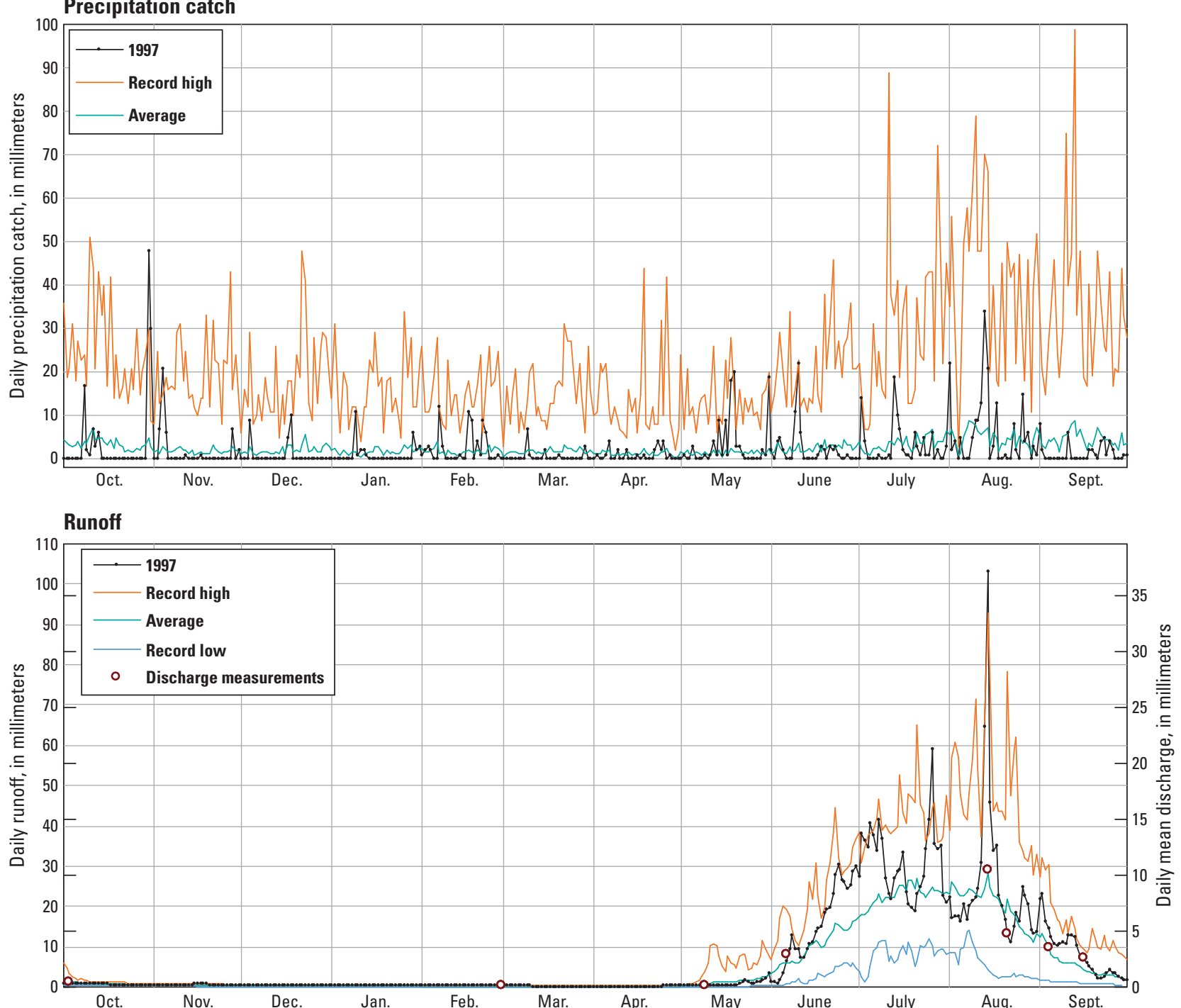

Figure 8. Daily values of mean air temperature, precipitation catch, and daily runoff at Phelan Creek, Alaska, water year 1997. 

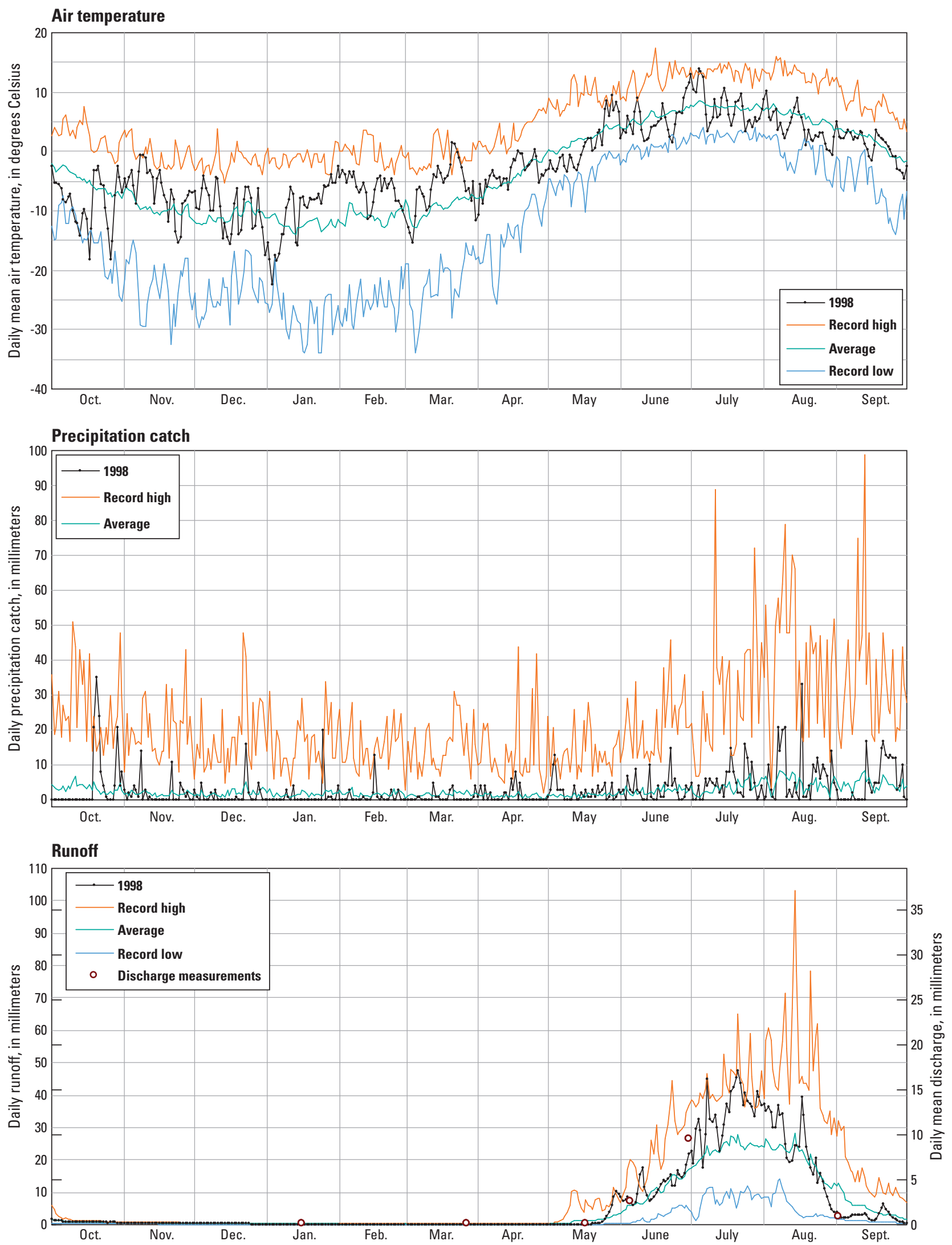

Figure 9. Daily values of mean air temperature, precipitation catch, and daily runoff at Phelan Creek, Alaska, water year 1998. 


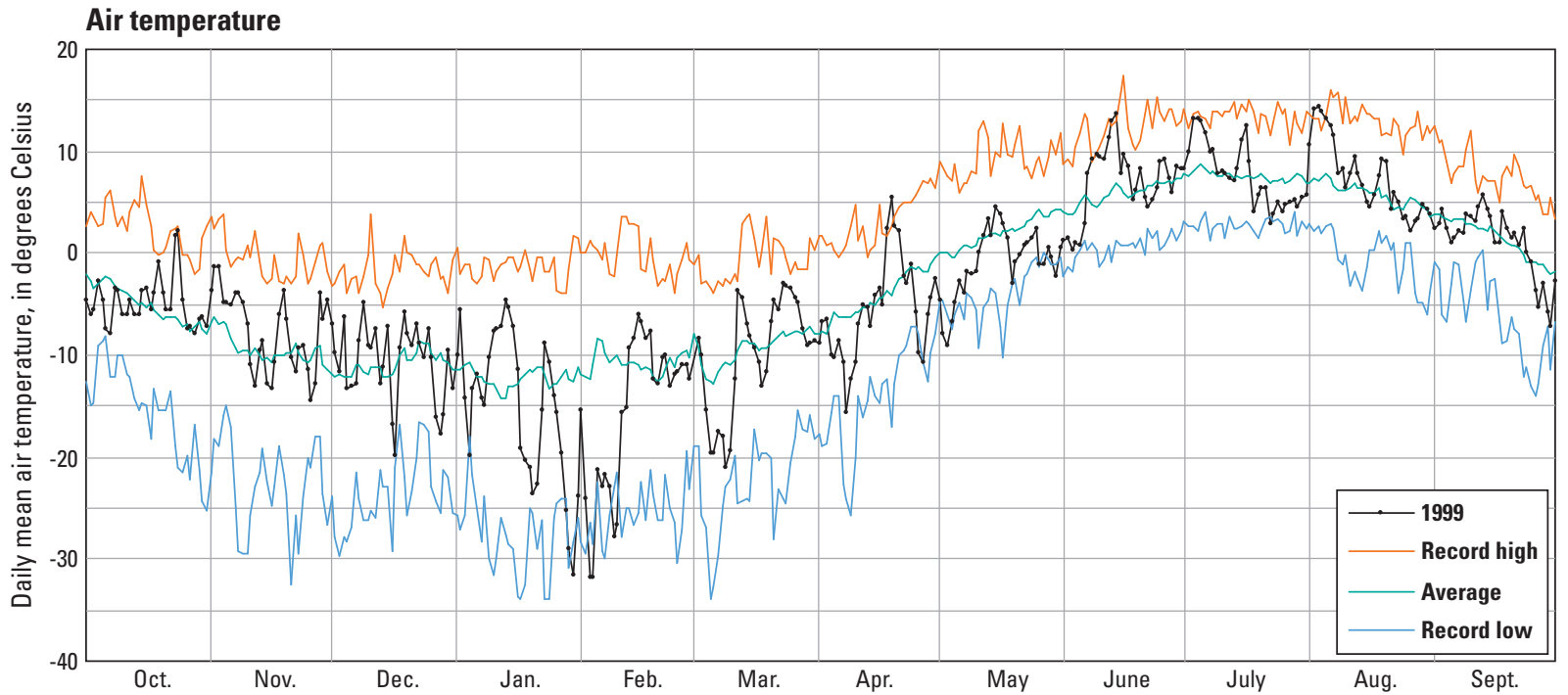

Precipitation catch
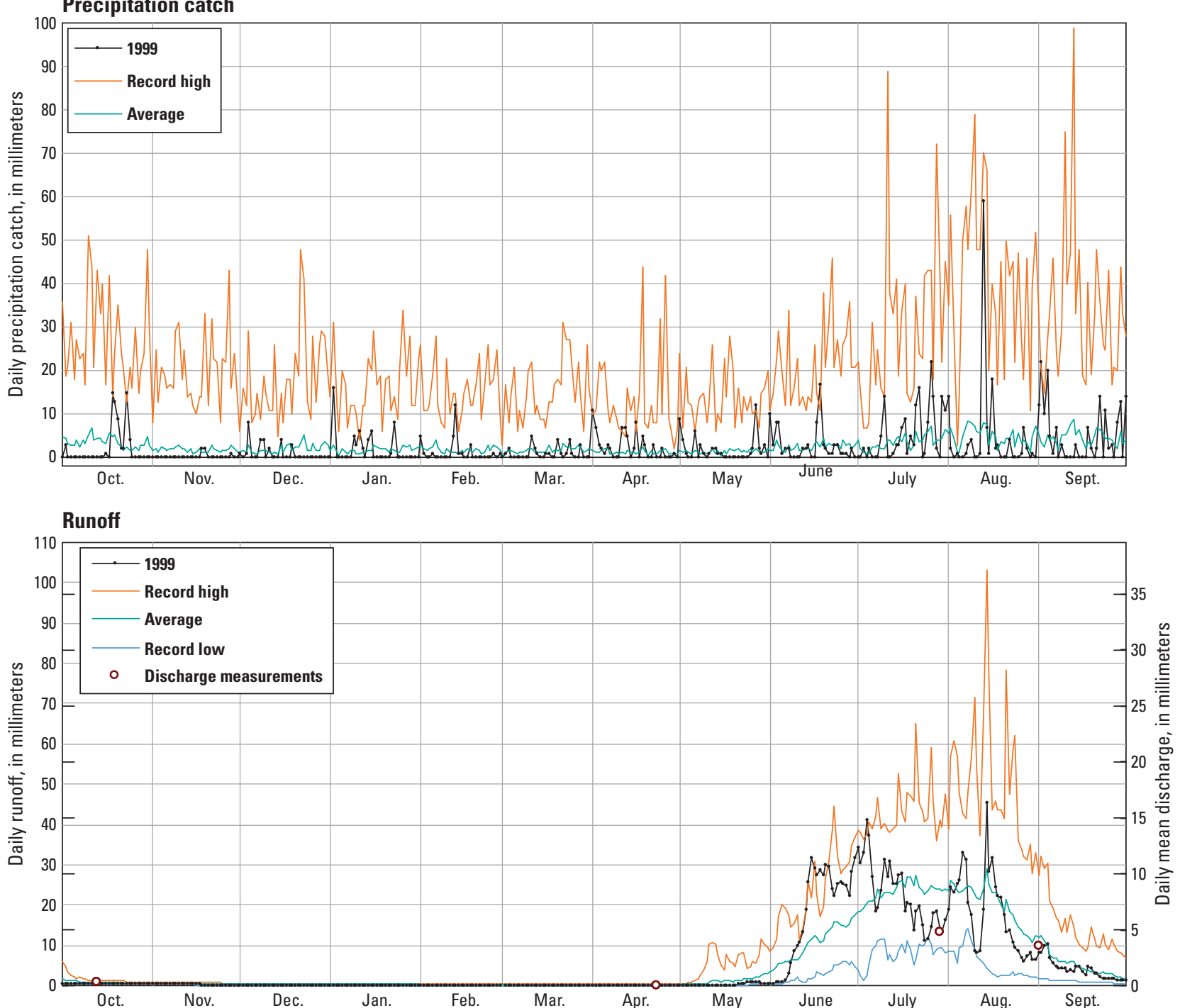

Figure 10. Daily values of mean air temperature, precipitation catch, and daily runoff at Phelan Creek, Alaska, water year 1999. 

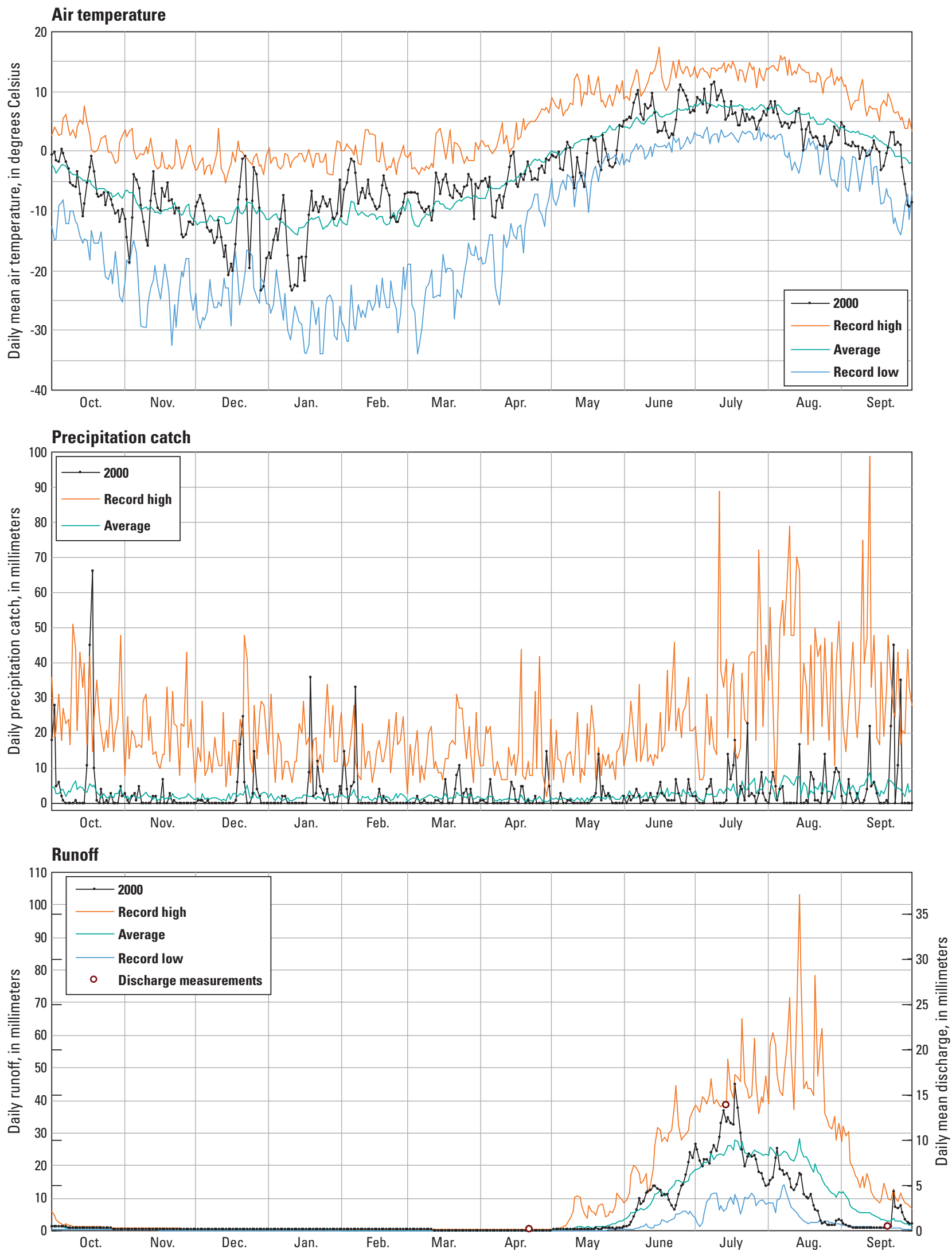

Figure 11. Daily values of mean air temperature, precipitation catch, and daily runoff at Phelan Creek, Alaska, water year 2000 

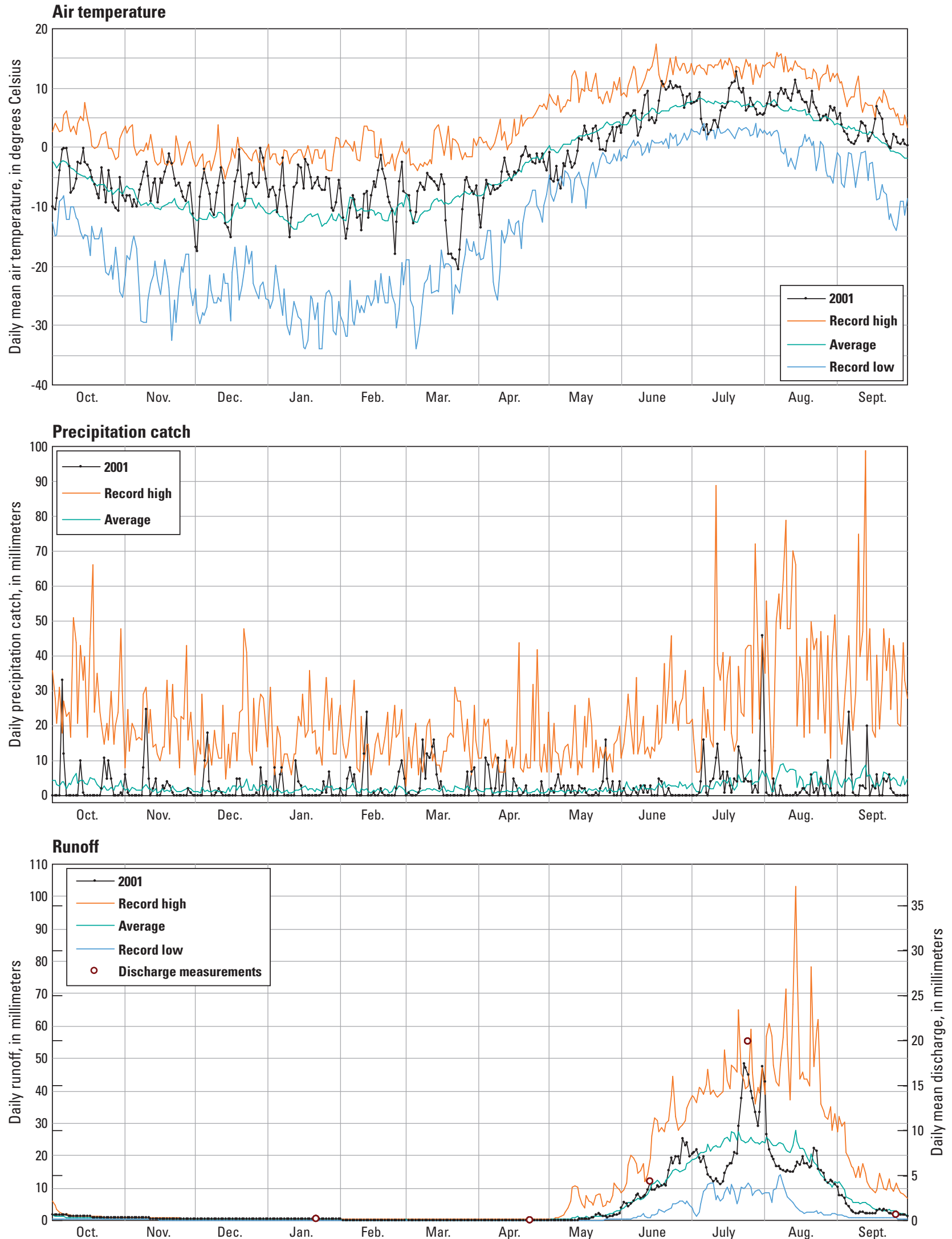

Figure 12. Daily values of mean air temperature, precipitation catch, and daily runoff at Phelan Creek, Alaska, water year 2001. 
Table 14. Daily, monthly, and annual average air temperature from analog sensor at 1,480 meters altitude, Gulkana Glacier basin, 1997 hydrologic year.

\begin{tabular}{|c|c|c|c|c|c|c|c|c|c|c|c|c|c|}
\hline \multicolumn{14}{|c|}{ Analog sensor } \\
\hline \multirow{3}{*}{ Day } & \multicolumn{12}{|c|}{ Air temperature, in degrees Celsius } & \multirow{3}{*}{ Annual } \\
\hline & \multicolumn{3}{|c|}{1996} & \multicolumn{9}{|c|}{1997} & \\
\hline & Oct. & Nov. & Dec. & Jan. & Feb. & Mar. & Apr. & May & Jun. & July & Aug. & Sept. & \\
\hline 1 & -6.5 & -5.8 & -14.9 & -22.1 & -10.3 & -15.7 & -9.6 & -1.0 & -1.5 & 10.5 & 5.9 & 2.9 & \\
\hline 2 & -8.0 & -8.7 & -17.2 & -21.4 & -7.8 & -17.1 & -11.6 & -1.2 & 2.3 & 10.6 & 6.7 & 3.1 & \\
\hline 3 & -6.0 & -9.8 & -10.9 & -17.2 & -5.6 & -19.4 & -8.5 & -1.6 & 6.6 & 9.5 & 8.5 & 4.2 & \\
\hline 4 & -5.1 & -6.6 & -9.8 & -18.7 & -4.4 & -17.0 & -4.9 & -1.5 & 9.3 & 9.5 & 9.6 & 5.5 & \\
\hline 5 & -4.5 & -9.7 & -17.2 & -17.4 & -2.9 & -10.4 & -1.9 & -1.2 & 8.9 & 7.4 & 9.2 & 3.7 & \\
\hline 6 & -3.9 & -11.2 & -20.3 & -25.9 & -3.8 & -8.9 & -0.2 & -3.8 & 6.5 & 8.6 & 5.2 & 3.6 & \\
\hline 7 & -3.7 & -12.6 & -10.9 & -28.8 & -6.4 & -11.5 & -1.9 & -1.6 & 0.6 & 9.4 & 7.2 & 4.6 & \\
\hline 8 & -3.0 & -15.3 & -12.5 & -18.2 & -9.2 & -10.8 & -2.0 & -0.7 & -0.1 & 9.7 & 6.5 & 4.2 & \\
\hline 9 & -4.3 & -15.1 & -10.8 & -5.2 & -5.9 & -13.9 & -2.8 & 0.2 & 0.5 & 6.8 & 6.1 & 2.9 & \\
\hline 10 & -8.9 & -5.9 & -14.1 & -4.8 & -6.0 & -18.4 & 1.8 & -1.1 & 2.5 & 7.6 & 6.8 & 3.4 & \\
\hline 11 & -8.4 & -2.8 & -13.3 & -6.1 & - 8.6 & -15.9 & 0.5 & -0.7 & 6.4 & 5.4 & 6.1 & 3.3 & \\
\hline 12 & -4.9 & -2.7 & -11.0 & -3.5 & -10.3 & -10.3 & -2.0 & 1.5 & 6.3 & 5.6 & 6.1 & 3.4 & \\
\hline 13 & -3.7 & -9.2 & -11.3 & -6.8 & -9.1 & -9.9 & -3.5 & -0.9 & 3.7 & 5.4 & 4.8 & 1.1 & \\
\hline 14 & -11.9 & -6.4 & -12.9 & -5.2 & -6.7 & -9.1 & -6.9 & -2.9 & 2.7 & 4.5 & 4.9 & 1.6 & \\
\hline 15 & -15.5 & -4.4 & -13.2 & -4.4 & -8.2 & -14.3 & -6.7 & -3.5 & 3.4 & 5.5 & 7.3 & 1.5 & \\
\hline 16 & -15.3 & -3.3 & -9.1 & -6.1 & -7.7 & -21.6 & -12.3 & -0.1 & 2.4 & 5.4 & 4.6 & 1.7 & \\
\hline 17 & -14.4 & -1.6 & -6.7 & -7.8 & -6.0 & -17.8 & -12.2 & -1.6 & 4.2 & 6.4 & 4.5 & 0.8 & \\
\hline 18 & -14.1 & -3.5 & -3.7 & -5.2 & -7.1 & -11.0 & -12.6 & -0.8 & 7.6 & 5.9 & 3.1 & -0.6 & \\
\hline 19 & -15.6 & -12.2 & -9.0 & -7.0 & -7.8 & -6.2 & -11.7 & 1.3 & 5.4 & 5.5 & 4.9 & -1.9 & \\
\hline 20 & -14.6 & -14.4 & -15.1 & -12.0 & -8.0 & -9.9 & -9.1 & 6.0 & 3.4 & 5.6 & 5.0 & -1.5 & \\
\hline 21 & -12.7 & -16.9 & -16.5 & -15.4 & -4.5 & -10.3 & -7.1 & 5.6 & 6.6 & 4.7 & 2.4 & 0.4 & \\
\hline 22 & -12.6 & -15.3 & -16.0 & -17.0 & -4.0 & -11.0 & -2.2 & 1.2 & 7.8 & 6.0 & 4.2 & 4.8 & \\
\hline 23 & -11.4 & -15.6 & -15.1 & -17.6 & -4.8 & -14.3 & 0.2 & -2.1 & 9.8 & 7.6 & 5.5 & 3.6 & \\
\hline 24 & -16.6 & -16.7 & -7.2 & -18.4 & -10.1 & -17.5 & 2.7 & -1.7 & 8.0 & 8.1 & 5.0 & 2.5 & \\
\hline 25 & -21.9 & -13.0 & -7.9 & -19.7 & -8.8 & -15.8 & 2.6 & -0.1 & 9.4 & 8.8 & 4.7 & -0.3 & \\
\hline 26 & -16.7 & -7.8 & -6.8 & -19.6 & -10.6 & -14.8 & 0.5 & 1.6 & 10.9 & 8.6 & 3.1 & 1.4 & \\
\hline 27 & -16.6 & -6.9 & -15.0 & -16.3 & -11.0 & -9.3 & -0.2 & 3.7 & 10.8 & 9.6 & 2.6 & -0.4 & \\
\hline 28 & -10.7 & -6.6 & -13.6 & -11.3 & -11.2 & -8.3 & -0.9 & 4.5 & 10.5 & 9.9 & 3.7 & -3.4 & \\
\hline 29 & -6.6 & -9.4 & -9.9 & -6.3 & & -11.4 & -0.7 & 5.7 & 10.7 & 9.3 & 6.1 & -2.7 & \\
\hline 30 & -4.9 & -9.9 & -21.2 & -7.7 & & -12.0 & -1.9 & 1.0 & 10.5 & 7.3 & 5.4 & -2.2 & \\
\hline 31 & -2.6 & -14.9 & -21.6 & -10.2 & & -10.7 & & -3.6 & & 4.8 & 5.2 & & \\
\hline $\begin{array}{c}\text { Monthly } \\
\text { average }\end{array}$ & -9.9 & -9.5 & -12.7 & -13.0 & -7.4 & -13.0 & -4.2 & 0.0 & 5.9 & 7.4 & 5.5 & 1.7 & -4.1 \\
\hline
\end{tabular}


Table 14. Daily, monthly, and annual average air temperature from digital sensor at 1,480 meters altitude, Gulkana Glacier basin, 1997 hydrologic year.-Continued

Digital sensor

\begin{tabular}{|c|c|c|c|c|c|c|c|c|c|c|c|c|c|}
\hline \multirow{2}{*}{ Day } & \multicolumn{12}{|c|}{ Air temperature, in degrees Celsius } & \multirow{2}{*}{ Annual } \\
\hline & Oct. & Nov. & Dec. & Jan. & Feb. & Mar. & Apr. & May & June & July & Aug. & Sept. & \\
\hline 1 & -6.1 & -5.3 & -13.7 & -21.1 & -9.6 & -14.4 & -8.0 & -0.9 & -0.5 & 11.6 & 7.3 & 4.1 & \\
\hline 2 & -7.3 & -8.0 & -16.5 & -20.7 & -6.6 & -16.5 & -10.6 & -0.5 & 3.0 & 12.0 & 8.1 & 4.3 & \\
\hline 3 & -5.3 & -8.7 & -9.7 & -16.2 & -4.3 & -19.0 & -7.5 & -0.0 & 7.2 & 10.9 & 9.6 & 5.6 & \\
\hline 5 & -4.5 & -9.2 & -16.5 & -16.4 & -2.1 & -10.5 & -1.3 & 0.1 & 9.5 & 9.0 & 10.5 & 5.1 & \\
\hline 6 & -3.3 & -10.7 & -19.5 & -25.1 & -2.9 & -8.3 & 0.3 & -2.1 & 7.6 & 10.0 & 7.0 & 5.0 & \\
\hline 7 & -2.8 & -12.1 & - 9.6 & -28.2 & -5.2 & -10.7 & -1.4 & -0.6 & 1.9 & 10.7 & 7.3 & 5.8 & \\
\hline 8 & -2.1 & -14.5 & -11.7 & -18.6 & -8.0 & -10.0 & -1.8 & -0.3 & 1.2 & 11.0 & 7.8 & 5.6 & \\
\hline 9 & -3.4 & -14.4 & -9.7 & -5.0 & -5.4 & -13.0 & -2.5 & 0.8 & 2.1 & 8.5 & 7.8 & 4.4 & \\
\hline 10 & -8.2 & -5.2 & -13.2 & -4.0 & -4.7 & -17.2 & 1.2 & 0.4 & 3.8 & 9.0 & 8.0 & 4.9 & \\
\hline 14 & -10.3 & -5.6 & -12.3 & -4.6 & -6.1 & -8.6 & -6.1 & -1.7 & 3.7 & 5.9 & 6.6 & 2.8 & \\
\hline 15 & -14.6 & -3.2 & -12.5 & -3.3 & -7.1 & -12.5 & -6.2 & -1.9 & 4.4 & 7.0 & 8.7 & 2.5 & \\
\hline 16 & -15.0 & -2.4 & -8.2 & -4.9 & -7.6 & -20.3 & -10.6 & 1.4 & 3.6 & 6.7 & 5.5 & 2.6 & \\
\hline 17 & -13.6 & -0.1 & -5.4 & -7.0 & -5.1 & -17.4 & -12.3 & -0.2 & 5.6 & 7.6 & 5.9 & 2.8 & \\
\hline 18 & -13.2 & -2.8 & - 3.1 & -4.1 & -6.2 & -11.3 & -14.2 & -0.5 & 8.5 & 7.6 & 4.4 & 1.4 & \\
\hline 19 & -15.3 & -11.0 & -7.8 & -6.1 & -7.4 & -5.2 & -10.9 & 3.4 & 6.5 & 6.8 & 5.9 & -0.6 & \\
\hline 20 & -14.4 & -13.6 & -14.0 & -10.7 & -7.2 & -9.0 & -9.2 & 6.3 & 5.1 & 7.1 & 6.4 & 0.1 & \\
\hline 21 & -12.2 & -16.3 & -15.6 & -14.6 & -3.8 & -10.0 & -6.0 & 6.3 & 7.9 & 6.2 & 4.0 & 2.0 & \\
\hline 22 & -12.0 & -14.4 & -14.8 & -16.0 & -2.8 & -10.5 & -1.4 & 2.9 & 9.2 & 7.3 & 5.8 & 6.0 & \\
\hline 23 & -10.7 & -14.5 & -13.6 & -17.1 & - 3.3 & -12.9 & 1.3 & -0.8 & 10.8 & 9.1 & 6.4 & 5.1 & \\
\hline 24 & -15.6 & -15.8 & -6.3 & -17.4 & -9.6 & -16.9 & 3.2 & -0.4 & 9.5 & 9.7 & 6.0 & 4.2 & \\
\hline $\begin{array}{l}\text { Monthly } \\
\text { average }\end{array}$ & -9.2 & - 8.6 & -11.7 & -12.2 & -6.6 & -12.5 & - 3.6 & 1.1 & 7.1 & 8.8 & 6.8 & 3.1 & - 3.1 \\
\hline $\begin{array}{c}\text { Departure } \\
\text { from normal }\end{array}$ & -4.2 & 0.3 & -1.0 & -0.4 & 4.1 & -2.9 & 0.5 & -0.9 & 1.1 & 1.2 & 1.0 & 1.6 & -0.1 \\
\hline $\begin{array}{l}\text { Standardized } \\
\text { value }\end{array}$ & -1.9 & 0.1 & -0.4 & -0.1 & 1.2 & -1.2 & 0.2 & -0.6 & 0.9 & 1.2 & 0.6 & 1.1 & -0.1 \\
\hline
\end{tabular}


Table 15. Daily, monthly, and annual average air temperature from analog sensor at 1,480 meters altitude, Gulkana Glacier basin, 1998 hydrologic year.

\begin{tabular}{|c|c|c|c|c|c|c|c|c|c|c|c|c|c|}
\hline \multicolumn{14}{|c|}{ Analog sensor } \\
\hline \multirow{3}{*}{ Day } & \multicolumn{12}{|c|}{ Air temperature, in degrees Celsius } & \multirow{3}{*}{ Annual } \\
\hline & \multicolumn{3}{|c|}{1997} & \multicolumn{9}{|c|}{1998} & \\
\hline & Oct. & Nov. & Dec. & Jan. & Feb. & Mar. & Apr. & May & June & July & Aug. & Sept. & \\
\hline 1 & -4.3 & -6.0 & -8.3 & -17.1 & -5.6 & -14.0 & -6.8 & -2.4 & 2.6 & 8.8 & 9.1 & 0.9 & \\
\hline 2 & -7.1 & -7.7 & -11.1 & -20.0 & -4.9 & -15.1 & -10.3 & -4.2 & 1.9 & 8.7 & 4.5 & 1.2 & \\
\hline 3 & -6.7 & -5.8 & -11.3 & -22.9 & -5.2 & -16.4 & -6.1 & -4.5 & 5.2 & 13.2 & 3.9 & 0.7 & \\
\hline 4 & -6.8 & -4.6 & -7.7 & -19.0 & -6.5 & -12.0 & -7.7 & -3.7 & 2.5 & 12.7 & 5.7 & 1.9 & \\
\hline 5 & -7.0 & -7.2 & -5.6 & -19.5 & -4.9 & -7.5 & -5.2 & -2.2 & 1.3 & 11.7 & 5.8 & 1.9 & \\
\hline 6 & -9.0 & -10.6 & -7.6 & -17.9 & -5.6 & -6.8 & -4.9 & -1.6 & 5.3 & 4.9 & 2.6 & 2.0 & \\
\hline 7 & -9.9 & -5.4 & -9.6 & -15.6 & -4.8 & -8.6 & -4.9 & -3.6 & 7.6 & 1.8 & 3.4 & 0.3 & \\
\hline 8 & -8.8 & -2.3 & -11.6 & -15.5 & -7.8 & -8.9 & -5.8 & -4.8 & 5.5 & 3.5 & 1.7 & 1.0 & \\
\hline 9 & -8.8 & -2.2 & -11.2 & -11.1 & - 8.7 & -11.4 & -5.2 & -2.0 & 2.5 & 4.3 & 1.0 & 2.8 & \\
\hline 10 & -11.5 & -2.4 & -5.8 & -9.8 & -8.2 & -9.6 & - 7.1 & -1.9 & 0.3 & 7.2 & 2.6 & 2.3 & \\
\hline 11 & -12.5 & -5.2 & -5.8 & -7.7 & -6.7 & - 7.9 & -5.8 & -3.7 & 0.9 & 4.5 & 3.7 & 2.0 & \\
\hline 12 & -13.4 & -4.9 & -10.7 & -8.8 & -8.5 & -6.0 & -5.7 & -3.7 & 1.2 & 4.8 & 4.8 & 0.3 & \\
\hline 13 & -15.6 & -5.8 & -16.1 & -16.9 & -12.5 & -5.2 & -8.0 & -3.7 & 2.8 & 7.7 & 6.2 & -0.9 & \\
\hline 14 & -11.8 & -10.1 & -14.1 & -16.7 & -12.2 & - 3.7 & -4.5 & -1.8 & 2.5 & 9.6 & 8.1 & -2.4 & \\
\hline 15 & -10.8 & -6.0 & -16.0 & - 9.6 & -10.1 & -4.8 & -2.6 & -0.9 & 3.3 & 7.1 & 5.2 & -2.8 & \\
\hline 16 & -13.0 & -4.6 & -17.1 & -10.3 & -7.7 & -6.8 & -2.7 & -0.1 & 3.7 & 5.2 & 4.1 & -0.3 & \\
\hline 17 & -19.3 & -6.7 & -15.4 & -10.6 & -6.1 & -5.8 & -2.4 & 1.4 & 4.4 & 2.9 & 2.1 & 2.2 & \\
\hline 18 & -14.1 & -8.7 & -10.7 & -10.8 & -6.2 & -5.8 & -1.0 & 2.2 & 7.0 & 4.8 & -0.3 & 0.7 & \\
\hline 19 & -4.6 & -10.5 & -8.0 & -9.5 & - 7.6 & -2.9 & -0.9 & -0.8 & 4.1 & 6.9 & 2.0 & 0.1 & \\
\hline 20 & -4.7 & -13.0 & -15.1 & -9.5 & -5.9 & 0.4 & -4.1 & -0.5 & 2.4 & 7.1 & 0.7 & -0.1 & \\
\hline 21 & -3.9 & -8.0 & -14.6 & -9.7 & - 7.3 & 0.3 & -3.4 & 2.1 & 0.9 & 8.3 & 0.4 & -0.1 & \\
\hline 22 & -7.0 & -9.8 & -9.9 & -9.1 & -6.1 & -1.1 & - 3.1 & 2.3 & -0.3 & 5.9 & 1.8 & -1.0 & \\
\hline 23 & -7.0 & -14.9 & -7.5 & -8.8 & -5.8 & -2.1 & -2.8 & 0.3 & 3.1 & 1.7 & 0.9 & -1.1 & \\
\hline 24 & -10.7 & -16.4 & -7.8 & -10.7 & -7.7 & -4.2 & -0.1 & 6.4 & 5.7 & 3.7 & 1.7 & -2.3 & \\
\hline 25 & -15.1 & -16.1 & - 9.8 & -7.4 & - 9.8 & -6.0 & -2.0 & 6.6 & 5.8 & 3.8 & 1.9 & -2.4 & \\
\hline 26 & -19.6 & -10.2 & -14.6 & -7.6 & -9.9 & -8.1 & -5.9 & 4.7 & 5.4 & 2.7 & 0.0 & -4.7 & \\
\hline 27 & -16.4 & -9.7 & -13.9 & -7.5 & -10.4 & -7.5 & -5.4 & 8.2 & 7.9 & 4.0 & -0.8 & -4.3 & \\
\hline 28 & -9.2 & -8.9 & -7.7 & -5.1 & -11.2 & -10.1 & -6.0 & 5.3 & 9.9 & 3.7 & -1.7 & -5.2 & \\
\hline 29 & -3.8 & -8.5 & -11.8 & -6.6 & & -6.2 & -4.8 & 7.3 & 10.2 & 3.9 & -2.3 & -6.3 & \\
\hline 30 & -5.8 & -7.9 & -14.3 & -7.1 & & -13.4 & -4.5 & 4.4 & 11.7 & 6.2 & 1.2 & - 3.8 & \\
\hline 31 & -8.7 & & -19.0 & -4.1 & & -12.6 & & 1.2 & & 7.8 & 3.3 & & \\
\hline $\begin{array}{l}\text { Monthly } \\
\text { average }\end{array}$ & -9.9 & -8.0 & -11.3 & -11.7 & -7.6 & -7.4 & -4.7 & 0.2 & 4.2 & 6.1 & 2.7 & -0.6 & -4.0 \\
\hline
\end{tabular}


Table 15. Daily, monthly, and annual average air temperature from digital sensor at 1,480 meters altitude, Gulkana Glacier basin, 1998 hydrologic year.-Continued

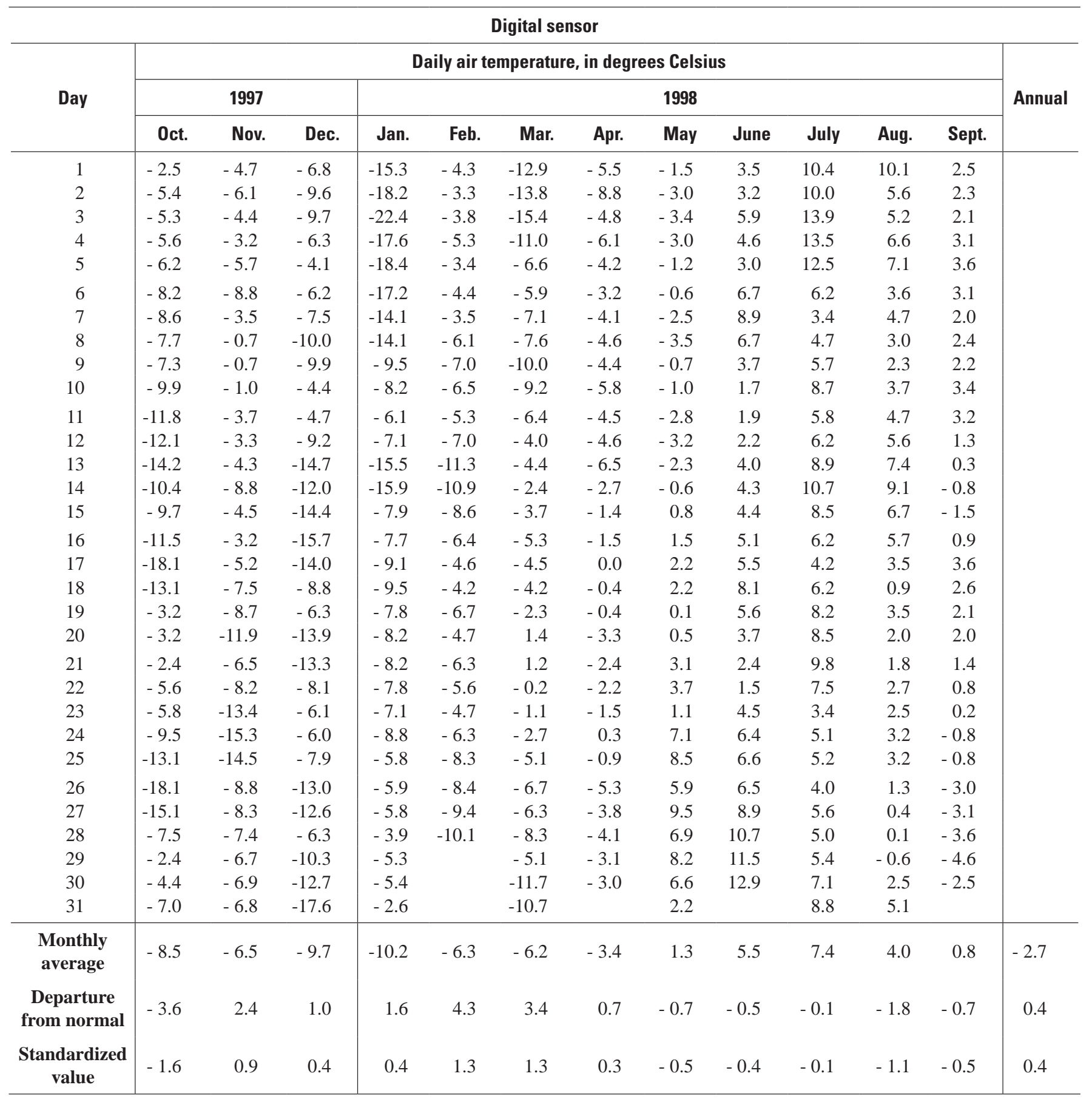


Table 16. Daily, monthly, and annual average air temperature from digital sensor at 1,480 meters altitude, Gulkana Glacier basin, 1999 hydrologic year.

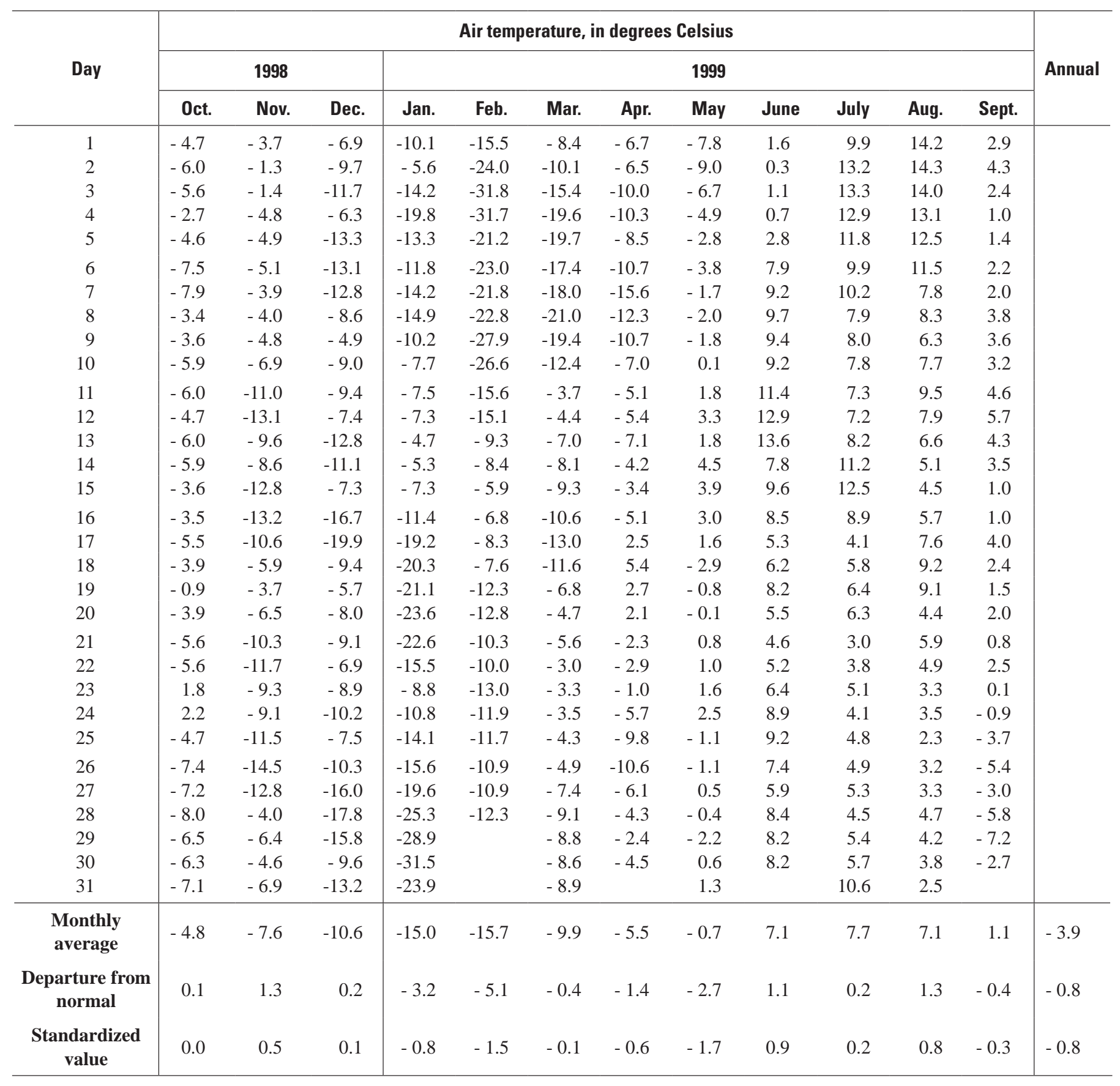


Table 17. Daily, monthly, and annual average air temperature from digital sensor at 1,480 meters altitude, Gulkana Glacier basin, 2000 hydrologic year.

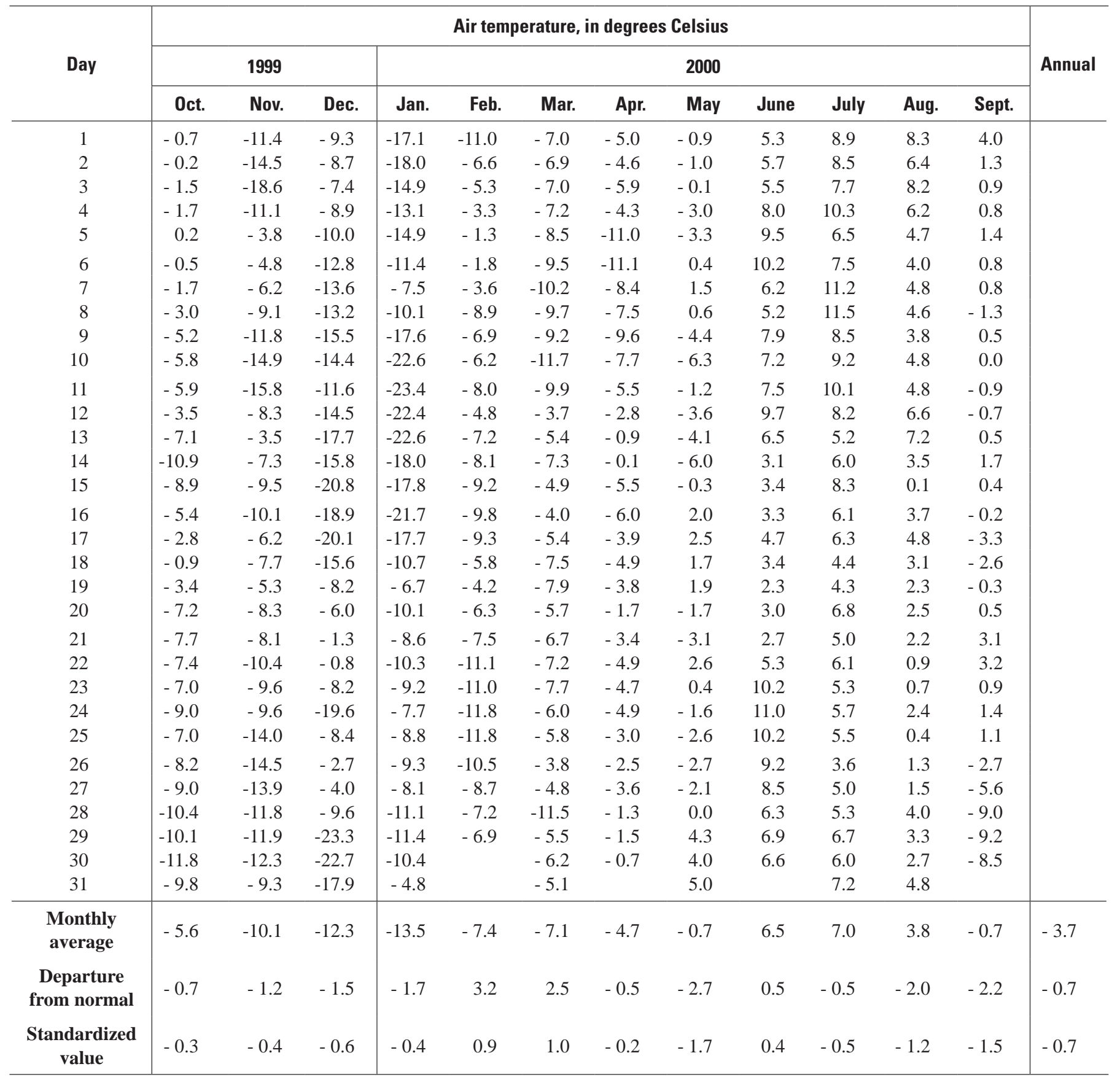


Table 18. Daily, monthly, and annual average air temperature from digital sensor at 1,480 meters altitude, Gulkana Glacier basin, 2001 hydrologic year.

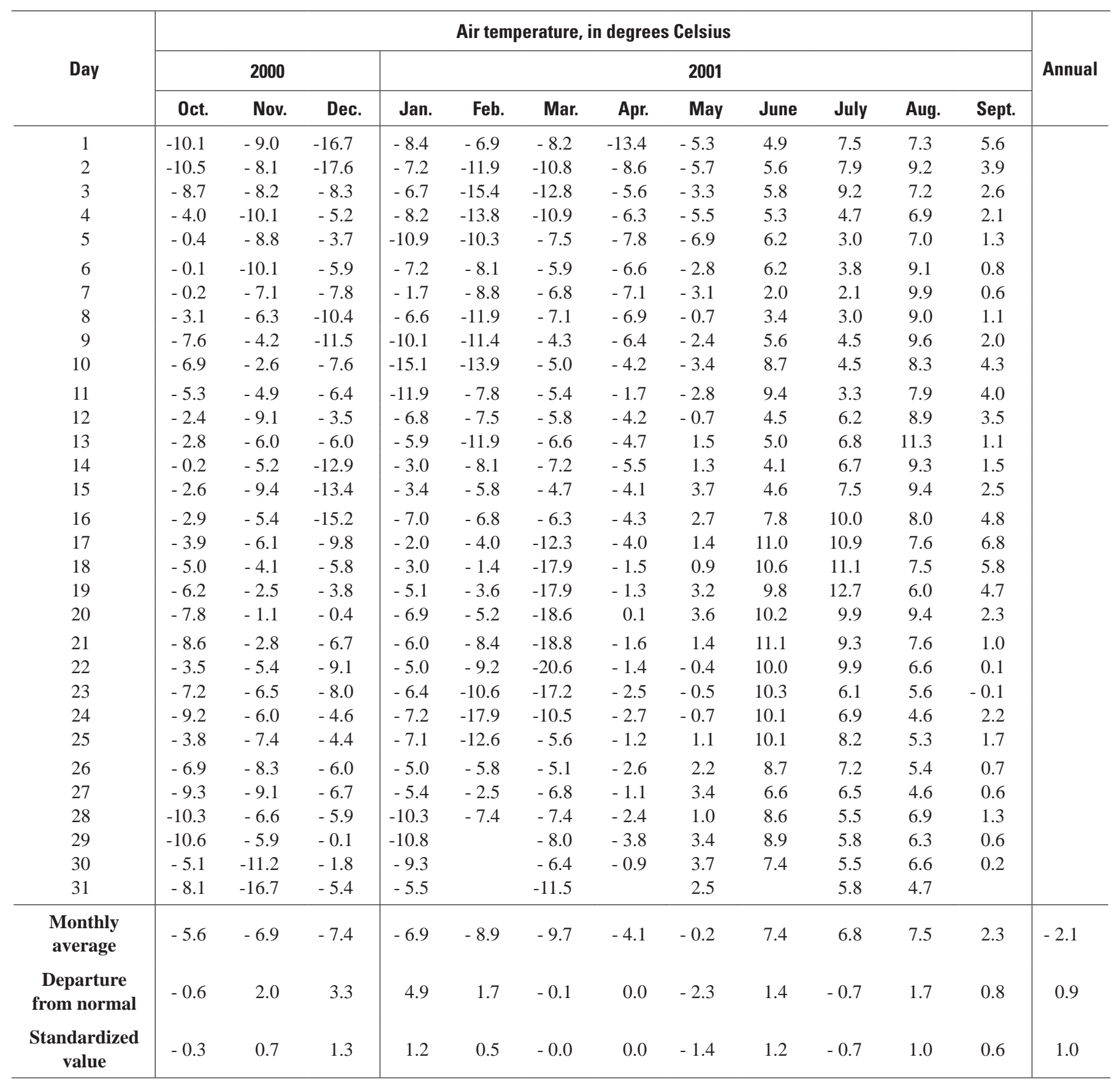

An analog temperature recorder with a slow time response and an accuracy of $1.0^{\circ} \mathrm{C}$ (Kennedy and others, 1997) has operated in its current configuration since the beginning of record in October 1967. After a 3-year overlap period, analysis of analog temperatures terminated in 1998, although data collection continued until spring 2007 as a backup. We expect differences between the analog sensor and the digital sensor due to the large difference in their time constants; a bias analysis for 1996-98 is shown in figure 13.
A storage-type precipitation gage with a modified Nipher wind shield (Kennedy and others, 1997) catches part of the total precipitation that falls at the weather-station site (tables 19-23). Daily precipitation catch has an estimated accuracy of $0.001 \mathrm{~m}$ and cumulative precipitation catch an accuracy of $0.002 \mathrm{~m}$ per month (Mayo and others, 1992; Kennedy, 1995). 
Water year 1996
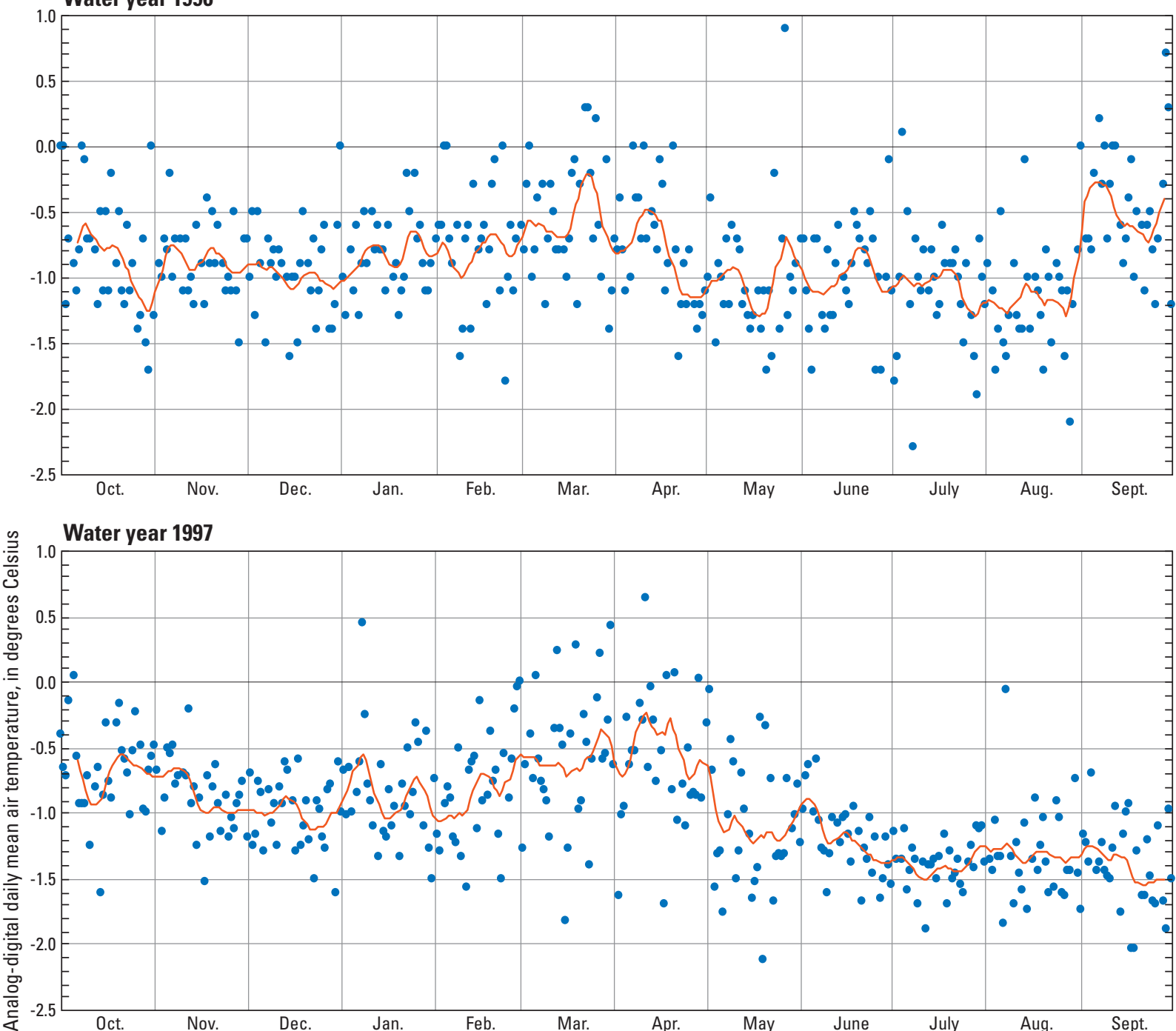

Water year 1998

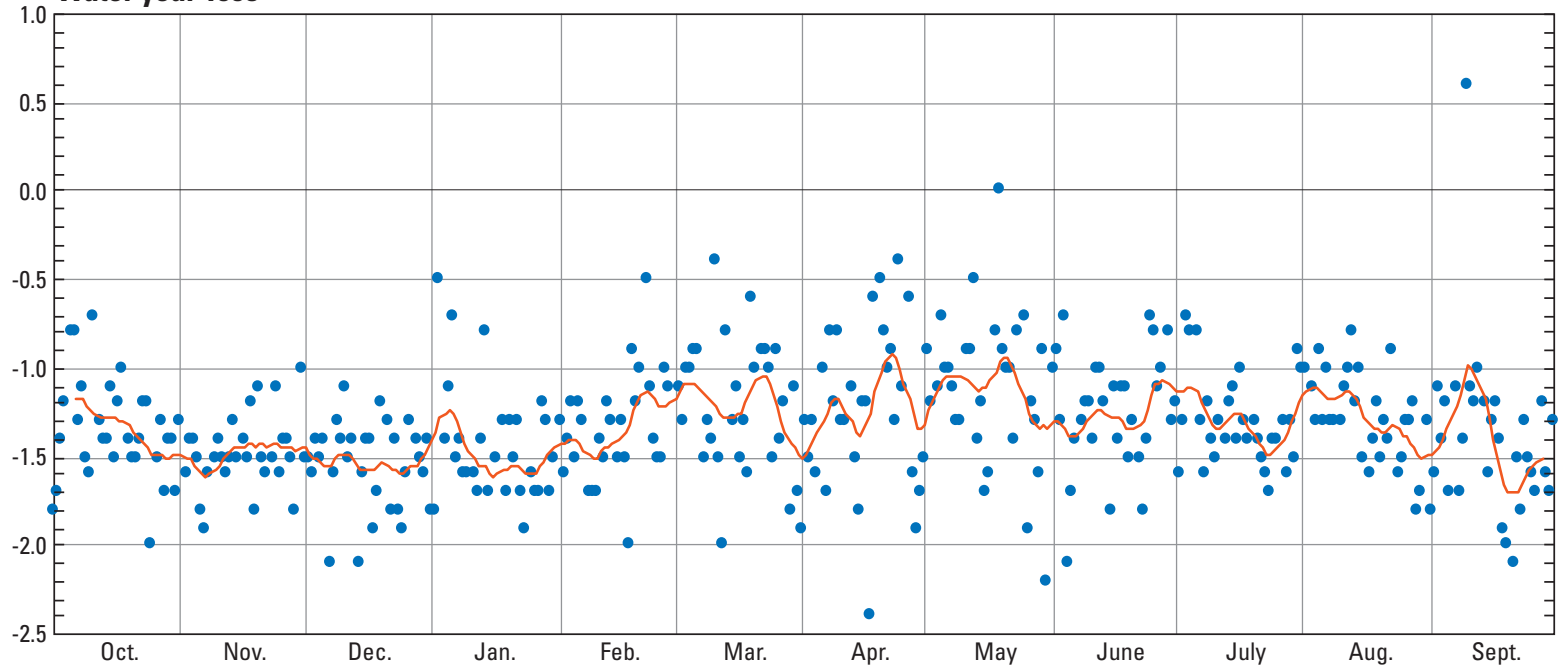

Figure 13. Difference between analog and digital temperature sensors at 1,480 meters altitude, Gulkana Glacier, Alaska, water years 1996-98. Red line is an inverse distance weighted smoothing of data with an 11-day window. 
Table 19. Daily, monthly, and annual precipitation catch recorded at 1,480 meters altitude, Gulkana Glacier basin, 1997 hydrologic year.

\begin{tabular}{|c|c|c|c|c|c|c|c|c|c|c|c|c|c|}
\hline \multirow{3}{*}{ Day } & \multicolumn{12}{|c|}{ Precipitation, in millimeters } & \multirow{3}{*}{ Annua } \\
\hline & \multicolumn{3}{|c|}{1996} & \multicolumn{9}{|c|}{1997} & \\
\hline & 0ct. & Nov. & Dec. & Jan. & Feb. & Mar. & Apr. & May & June & July & Aug. & Sept. & \\
\hline 1 & 0 & 0 & 0 & 0 & 1 & 0 & 1 & 1 & 0 & 14 & 2 & 2 & \\
\hline 2 & 0 & 0 & 0 & 0 & 2 & 0 & 0 & 0 & 4 & 4 & 4 & 0 & \\
\hline 3 & 0 & 7 & 0 & 0 & 3 & 0 & 0 & 0 & 5 & 0 & 0 & 0 & \\
\hline 4 & 0 & 21 & 9 & 0 & 2 & 0 & 1 & 3 & 2 & 0 & 5 & 0 & \\
\hline 5 & 0 & 6 & 0 & 0 & 0 & 0 & 4 & 0 & 0 & 0 & 0 & 0 & \\
\hline 6 & 0 & 0 & 0 & 0 & 0 & 0 & 0 & 0 & 0 & 0 & 0 & 0 & \\
\hline 7 & 0 & 0 & 0 & 0 & 12 & 0 & 0 & 0 & 0 & 1 & 0 & 0 & \\
\hline 8 & 17 & 0 & 0 & 0 & 3 & 7 & 2 & 1 & 11 & 0 & 5 & 0 & \\
\hline 9 & 2 & 0 & 0 & 11 & 0 & 1 & 0 & 0 & 22 & 0 & 9 & 0 & \\
\hline 10 & 1 & 0 & 0 & 1 & 0 & 0 & 0 & 1 & 6 & 1 & 9 & 6 & \\
\hline 11 & 7 & 0 & 0 & 2 & 0 & 0 & 2 & 4 & 0 & 0 & 13 & 0 & \\
\hline 12 & 3 & 1 & 0 & 2 & 0 & 0 & 0 & 1 & 0 & 19 & 34 & 0 & \\
\hline 13 & 6 & 0 & 0 & 1 & 0 & 0 & 0 & 9 & 0 & 10 & 21 & 0 & \\
\hline 14 & 0 & 0 & 0 & 0 & 1 & 2 & 0 & 1 & 0 & 7 & 0 & 0 & \\
\hline 15 & 0 & 0 & 0 & 0 & 0 & 0 & 1 & 9 & 0 & 2 & 3 & 0 & \\
\hline 16 & 0 & 0 & 0 & 0 & 0 & 0 & 0 & 1 & 1 & 1 & 13 & 0 & \\
\hline 17 & 0 & 1 & 5 & 0 & 11 & 0 & 1 & 18 & 3 & 1 & 0 & 2 & \\
\hline 18 & 0 & 0 & 10 & 0 & 9 & 0 & 0 & 20 & 2 & 0 & 0 & 2 & \\
\hline 19 & 0 & 0 & 0 & 0 & 0 & 1 & 0 & 3 & 0 & 6 & 1 & 1 & \\
\hline 20 & 0 & 0 & 0 & 0 & 4 & 0 & 0 & 3 & 3 & 3 & 0 & 0 & \\
\hline 21 & 0 & 0 & 0 & 0 & 1 & 0 & 2 & 1 & 2 & 1 & 0 & 4 & \\
\hline 22 & 0 & 0 & 0 & 0 & 9 & 0 & 4 & 0 & 3 & 5 & 8 & 5 & \\
\hline 23 & 0 & 0 & 0 & 0 & 6 & 0 & 2 & 0 & 1 & 1 & 2 & 1 & \\
\hline 24 & 0 & 0 & 0 & 0 & 0 & 0 & 4 & 0 & 0 & 1 & 0 & 4 & \\
\hline 25 & 0 & 0 & 0 & 0 & 0 & 0 & 0 & 0 & 0 & 6 & 15 & 2 & \\
\hline 26 & 0 & 0 & 0 & 0 & 0 & 0 & 1 & 0 & 1 & 0 & 4 & 0 & \\
\hline 27 & 0 & 0 & 0 & 0 & 1 & 0 & 0 & 0 & 0 & 2 & 6 & 0 & \\
\hline 28 & 0 & 7 & 0 & 0 & 0 & 3 & 0 & 2 & 0 & 0 & 0 & 0 & \\
\hline 29 & 0 & 0 & 0 & 6 & & 0 & 0 & 1 & 0 & 0 & 3 & 1 & \\
\hline 30 & 48 & 2 & 0 & 2 & & 0 & 0 & 19 & 0 & 3 & 1 & 1 & \\
\hline 31 & 30 & 0 & 0 & 3 & & 0 & & 2 & & 22 & 8 & & \\
\hline Total & 114 & 45 & 24 & 28 & 65 & 14 & 25 & 100 & 66 & 110 & 166 & 31 & 788 \\
\hline $\begin{array}{l}\text { Departure } \\
\text { from normal }\end{array}$ & 3 & -13 & -42 & -29 & 11 & -46 & -17 & 52 & -16 & -10 & 18 & -107 & -195 \\
\hline $\begin{array}{l}\text { Standardized } \\
\text { value }\end{array}$ & 0.1 & -0.3 & -1.0 & -1.0 & 0.3 & -1.2 & -0.7 & 2.2 & -0.4 & -0.1 & 0.2 & -1.3 & -0.8 \\
\hline
\end{tabular}


Table 20. Daily, monthly, and annual precipitation catch recorded at 1,480 meters altitude, Gulkana Glacier basin, 1998 hydrologic year.

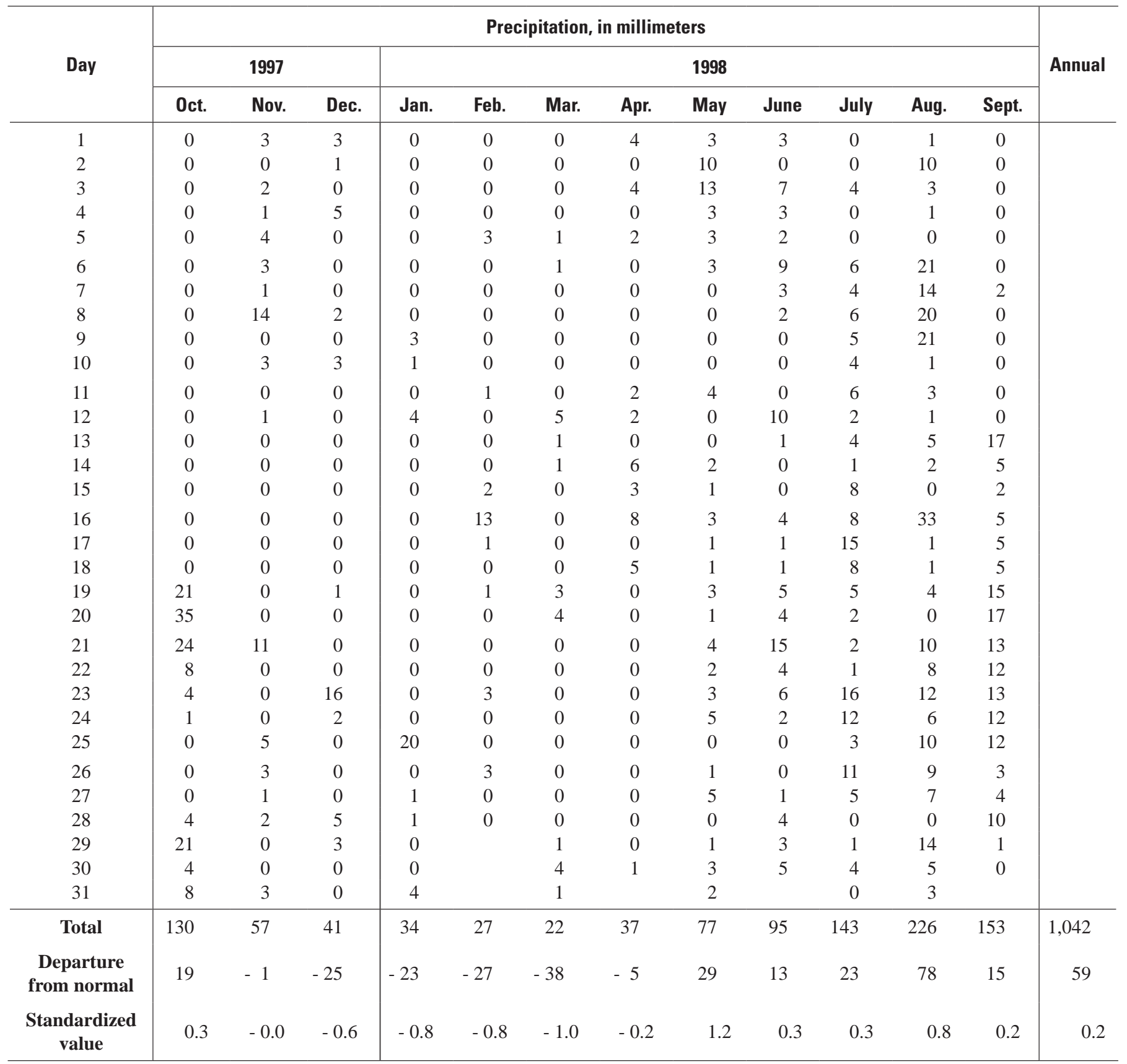


Table 21. Daily, monthly, and annual precipitation catch recorded at 1,480 meters altitude, Gulkana Glacier basin, 1999 hydrologic year.

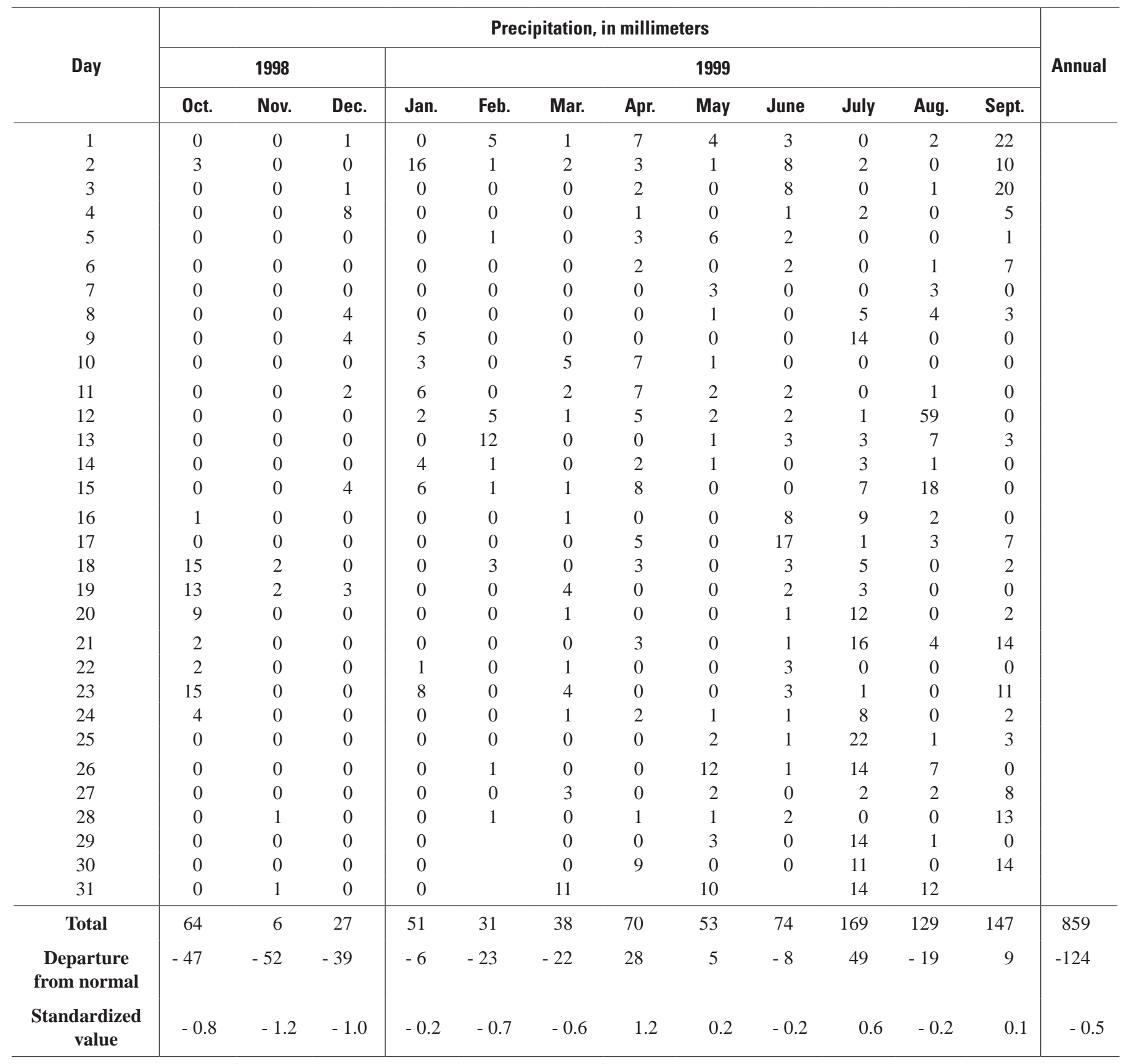


Table 22. Daily, monthly, and annual precipitation catch recorded at 1,480 meters altitude, Gulkana Glacier basin, 2000 hydrologic year.

\begin{tabular}{|c|c|c|c|c|c|c|c|c|c|c|c|c|c|}
\hline \multirow[b]{2}{*}{ Day } & \multicolumn{12}{|c|}{ Precipitation, in millimeters } & \multirow[b]{2}{*}{ Annual } \\
\hline & \multicolumn{3}{|c|}{1999} & \multicolumn{9}{|c|}{2000} & \\
\hline 1 & 18 & 3 & 0 & 0 & 3 & 0 & 1 & 0 & 0 & 1 & 3 & 0 & \\
\hline 2 & 28 & 1 & 1 & 0 & 15 & 0 & 0 & 0 & 2 & 0 & 9 & 0 & \\
\hline 3 & 5 & 0 & 1 & 0 & 4 & 0 & 0 & 0 & 1 & 0 & 5 & 7 & \\
\hline 5 & 2 & 3 & 0 & 0 & 5 & 0 & 0 & 0 & 4 & 4 & 4 & 0 & \\
\hline 6 & 1 & 1 & 1 & 0 & 6 & 0 & 0 & 0 & 2 & 5 & 5 & 1 & \\
\hline 7 & 0 & 5 & 0 & 2 & 33 & 1 & 0 & 1 & 0 & 7 & 0 & 3 & \\
\hline 8 & 0 & 0 & 0 & 2 & 0 & 0 & 0 & 1 & 1 & 0 & 0 & 0 & \\
\hline 9 & 0 & 0 & 0 & 0 & 0 & 0 & 0 & 0 & 1 & 0 & 0 & 0 & \\
\hline 10 & 0 & 0 & 0 & 0 & 0 & 0 & 0 & 0 & 1 & 0 & 0 & 1 & \\
\hline 14 & 0 & 2 & 0 & 0 & 0 & 0 & 4 & 0 & 3 & 14 & 0 & 6 & \\
\hline 15 & 0 & 0 & 0 & 0 & 0 & 0 & 1 & 0 & 6 & 7 & 0 & 3 & \\
\hline 16 & 11 & 0 & 0 & 0 & 0 & 7 & 0 & 0 & 3 & 11 & 1 & 0 & \\
\hline 17 & 45 & 7 & 0 & 0 & 4 & 0 & 5 & 1 & 2 & 18 & 0 & 0 & \\
\hline 18 & 66 & 0 & 1 & 9 & 0 & 0 & 5 & 2 & 1 & 0 & 9 & 0 & \\
\hline 19 & 10 & 0 & 6 & 36 & 2 & 0 & 0 & 3 & 1 & 5 & 7 & 1 & \\
\hline 20 & 1 & 3 & 17 & 2 & 1 & 4 & 1 & 14 & 1 & 4 & 1 & 0 & \\
\hline 21 & 0 & 0 & 25 & 3 & 0 & 8 & 0 & 1 & 1 & 1 & 1 & 22 & \\
\hline 22 & 4 & 1 & 6 & 12 & 0 & 11 & 0 & 5 & 7 & 23 & 0 & 45 & \\
\hline 23 & 0 & 0 & 0 & 5 & 0 & 0 & 2 & 2 & 3 & 2 & 3 & 0 & \\
\hline 24 & 1 & 0 & 0 & 3 & 0 & 3 & 0 & 3 & 0 & 3 & 14 & 11 & \\
\hline Total & 209 & 30 & 83 & 84 & 73 & 47 & 52 & 40 & 61 & 124 & 114 & 169 & 1,086 \\
\hline $\begin{array}{c}\text { Departure } \\
\text { from normal }\end{array}$ & 98 & -28 & 17 & 27 & 19 & -13 & 10 & -8 & -21 & 4 & -34 & 31 & 103 \\
\hline $\begin{array}{l}\text { Standardized } \\
\text { value }\end{array}$ & 1.7 & -0.6 & 0.4 & 0.9 & 0.6 & -0.3 & 0.4 & -0.4 & -0.5 & 0.0 & -0.4 & 0.4 & 0.4 \\
\hline
\end{tabular}


Table 23. Daily, monthly, and annual precipitation catch recorded at 1,480 meters altitude, Gulkana Glacier basin, 2001 hydrologic year.

\begin{tabular}{|c|c|c|c|c|c|c|c|c|c|c|c|c|c|}
\hline \multirow{3}{*}{ Day } & \multicolumn{12}{|c|}{ Precipitation, in millimeters } & \multirow{3}{*}{ Annual } \\
\hline & \multicolumn{3}{|c|}{2000} & \multicolumn{9}{|c|}{2001} & \\
\hline & Oct. & Nov. & Dec. & Jan. & Feb. & Mar. & Apr. & May & June & July & Aug. & Sept. & \\
\hline 1 & 0 & 6 & 0 & 0 & 2 & 1 & 0 & 2 & 2 & 0 & 1 & 0 & \\
\hline 2 & 0 & 1 & 0 & 0 & 0 & 0 & 0 & 1 & 0 & 0 & 0 & 0 & \\
\hline 3 & 0 & 0 & 0 & 0 & 0 & 0 & 11 & 3 & 0 & 1 & 0 & 0 & \\
\hline 4 & 0 & 0 & 0 & 8 & 5 & 0 & 9 & 6 & 0 & 4 & 5 & 10 & \\
\hline 5 & 33 & 0 & 10 & 0 & 8 & 1 & 2 & 2 & 2 & 16 & 0 & 24 & \\
\hline 6 & 12 & 0 & 18 & 6 & 4 & 2 & 0 & 3 & 1 & 1 & 0 & 6 & \\
\hline 7 & 0 & 0 & 4 & 8 & 6 & 16 & 2 & 0 & 0 & 0 & 3 & 1 & \\
\hline 8 & 0 & 0 & 0 & 0 & 0 & 5 & 11 & 3 & 3 & 4 & 0 & 0 & \\
\hline 9 & 0 & 8 & 0 & 0 & 0 & 12 & 0 & 1 & 1 & 5 & 0 & 0 & \\
\hline 10 & 0 & 25 & 1 & 0 & 0 & 11 & 3 & 3 & 3 & 11 & 0 & 3 & \\
\hline 11 & 0 & 5 & 2 & 0 & 12 & 14 & 10 & 0 & 1 & 15 & 0 & 3 & \\
\hline 12 & 0 & 0 & 1 & 0 & 24 & 16 & 0 & 1 & 3 & 5 & 0 & 2 & \\
\hline 13 & 10 & 2 & 0 & 10 & 0 & 6 & 0 & 3 & 0 & 7 & 1 & 20 & \\
\hline 14 & 1 & 5 & 0 & 4 & 0 & 2 & 0 & 2 & 0 & 1 & 0 & 0 & \\
\hline 15 & 0 & 0 & 0 & 3 & 1 & 4 & 5 & 2 & 0 & 7 & 1 & 3 & \\
\hline 16 & 0 & 0 & 0 & 0 & 0 & 0 & 3 & 1 & 5 & 3 & 2 & 2 & \\
\hline 17 & 0 & 3 & 0 & 0 & 0 & 0 & 0 & 0 & 4 & 1 & 2 & 6 & \\
\hline 18 & 0 & 2 & 0 & 0 & 2 & 0 & 0 & 0 & 0 & 5 & 1 & 0 & \\
\hline 19 & 0 & 4 & 5 & 0 & 1 & 0 & 1 & 0 & 0 & 4 & 0 & 0 & \\
\hline 20 & 0 & 3 & 5 & 0 & 0 & 0 & 3 & 1 & 2 & 14 & 6 & 5 & \\
\hline 21 & 0 & 1 & 0 & 0 & 0 & 0 & 0 & 0 & 0 & 11 & 1 & 4 & \\
\hline 22 & 2 & 0 & 0 & 0 & 0 & 0 & 0 & 2 & 0 & 4 & 2 & 6 & \\
\hline 23 & 11 & 0 & 0 & 0 & 0 & 0 & 0 & 4 & 0 & 5 & 0 & 2 & \\
\hline 24 & 5 & 0 & 0 & 1 & 0 & 0 & 0 & 16 & 0 & 4 & 5 & 1 & \\
\hline 25 & 10 & 0 & 0 & 4 & 2 & 0 & 0 & 5 & 0 & 4 & 2 & 0 & \\
\hline 26 & 5 & 0 & 0 & 1 & 7 & 7 & 0 & 1 & 0 & 1 & 0 & 0 & \\
\hline 27 & 0 & 0 & 1 & 7 & 10 & 0 & 4 & 4 & 0 & 3 & 10 & 0 & \\
\hline 28 & 0 & 2 & 0 & 0 & 5 & 7 & 0 & 0 & 0 & 1 & 2 & 0 & \\
\hline 29 & 0 & 1 & 8 & 0 & & 8 & 0 & 0 & 0 & 10 & 0 & 0 & \\
\hline 30 & 1 & 0 & 0 & 0 & & 2 & 0 & 4 & 0 & 46 & 0 & 0 & \\
\hline 31 & 0 & 0 & 4 & 0 & & 0 & & 0 & & 13 & 1 & & \\
\hline Total & 90 & 68 & 59 & 52 & 89 & 114 & 64 & 70 & 27 & 206 & 45 & 98 & 982 \\
\hline $\begin{array}{l}\text { Departure } \\
\text { from normal }\end{array}$ & -21 & 10 & -7 & -5 & 35 & 54 & 22 & 22 & -55 & 86 & -103 & -40 & -1 \\
\hline $\begin{array}{l}\text { Standardized } \\
\text { value }\end{array}$ & -0.4 & 0.2 & -0.2 & -0.2 & 1.1 & 1.3 & 0.9 & 0.9 & -1.3 & 1.0 & -1.1 & -0.5 & -0.0 \\
\hline
\end{tabular}


Prior to 1996, precipitation catch data were processed with a temperature and water-concentration dependent density correction. However, analysis that was not possible at the beginning of the monitoring program indicates that the density correction is incomplete and actually increases the measurement error rather than reducing it. Hence, the density correction was not applied to data after 1995. Comparison of density-corrected data to non-density-corrected data for earlier years shows monthly and annual differences of several percent. These differences are judged to be minor compared to other errors. Daily value differences can be greater, with precipitation occasionally shifted from 1 day to an adjacent day.

A Taylor Scientific Engineering WS-3 rotor anemometer, designed for a very windy, rugged mountain environment, records 3-m height wind speeds. The threshold wind speed is $1.34 \mathrm{~m} / \mathrm{s}$ and it has an accuracy of about $0.5 \mathrm{~m} / \mathrm{s}$. The time constant for a step increase in wind speed is 7-8 seconds and 19-20 seconds for a step decrease. Fifteen-minute Average wind speeds are recorded (fig. 14, tables 24-28).

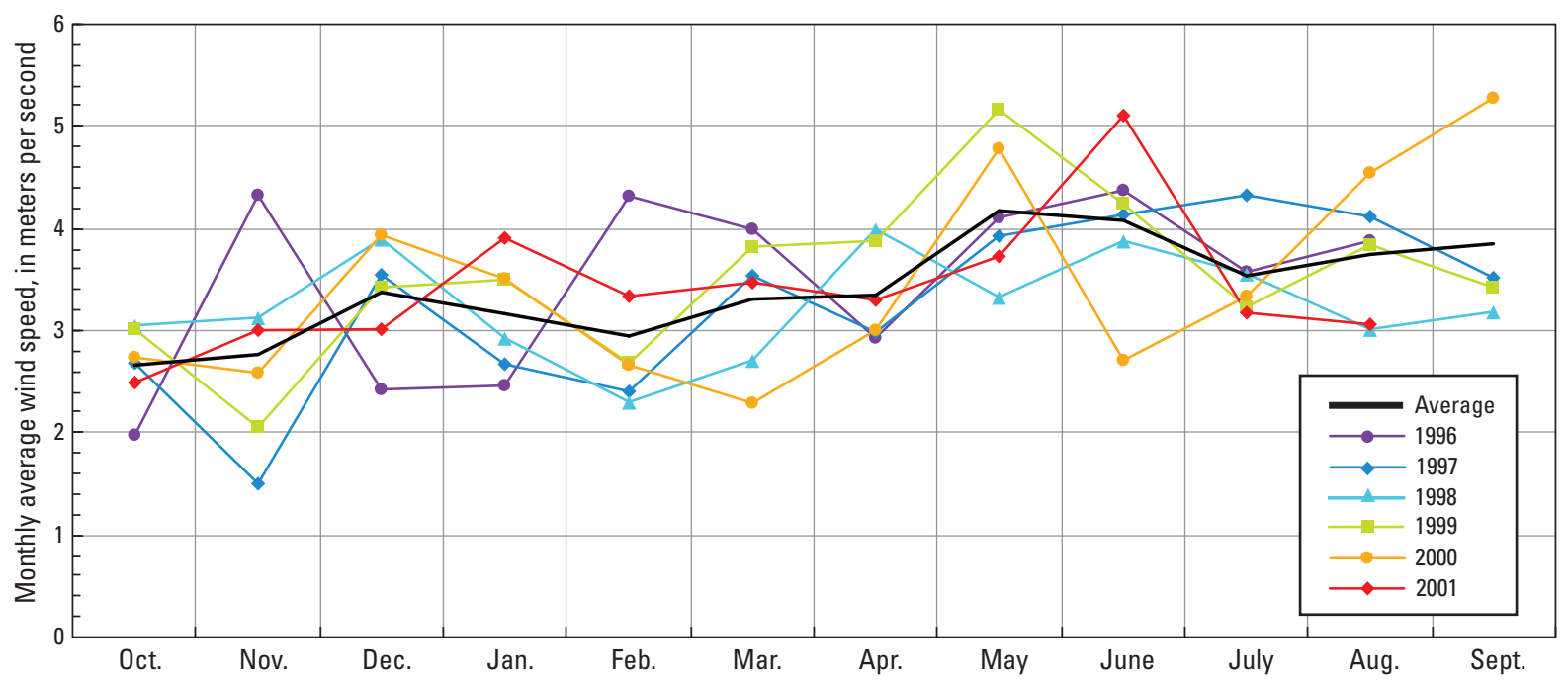

Figure 14. Monthly average wind speed at 1,480 meters altitude, Gulkana Glacier basin, Alaska, water years 1997-2001. 
Table 24. Daily, monthly, and annual average wind speed at 1,480 meters altitude, Gulkana Glacier basin, 1997 hydrologic year.

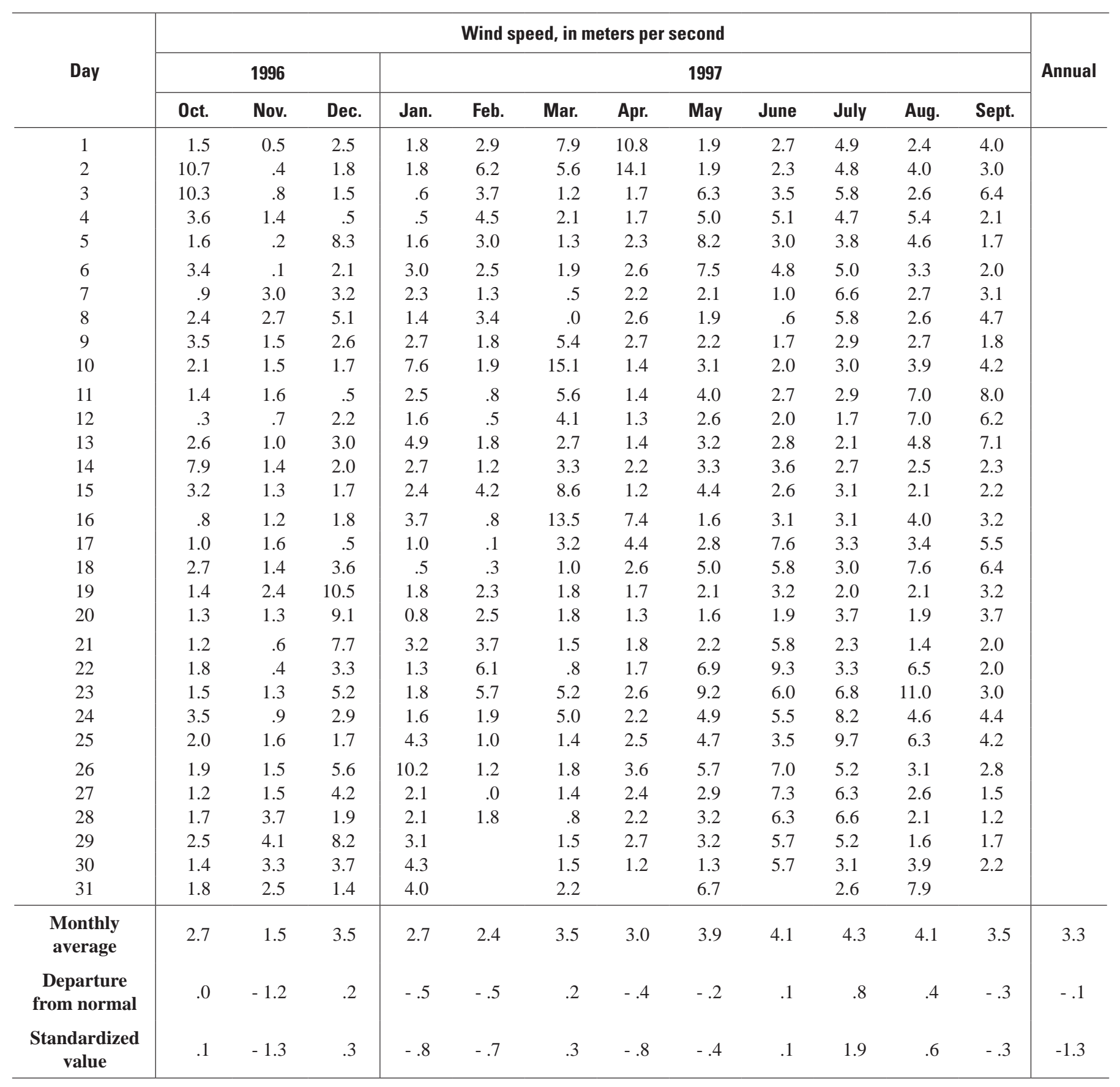


Table 25. Daily, monthly, and annual average wind speed at 1,480 meters altitude, Gulkana Glacier basin, 1998 hydrologic year.

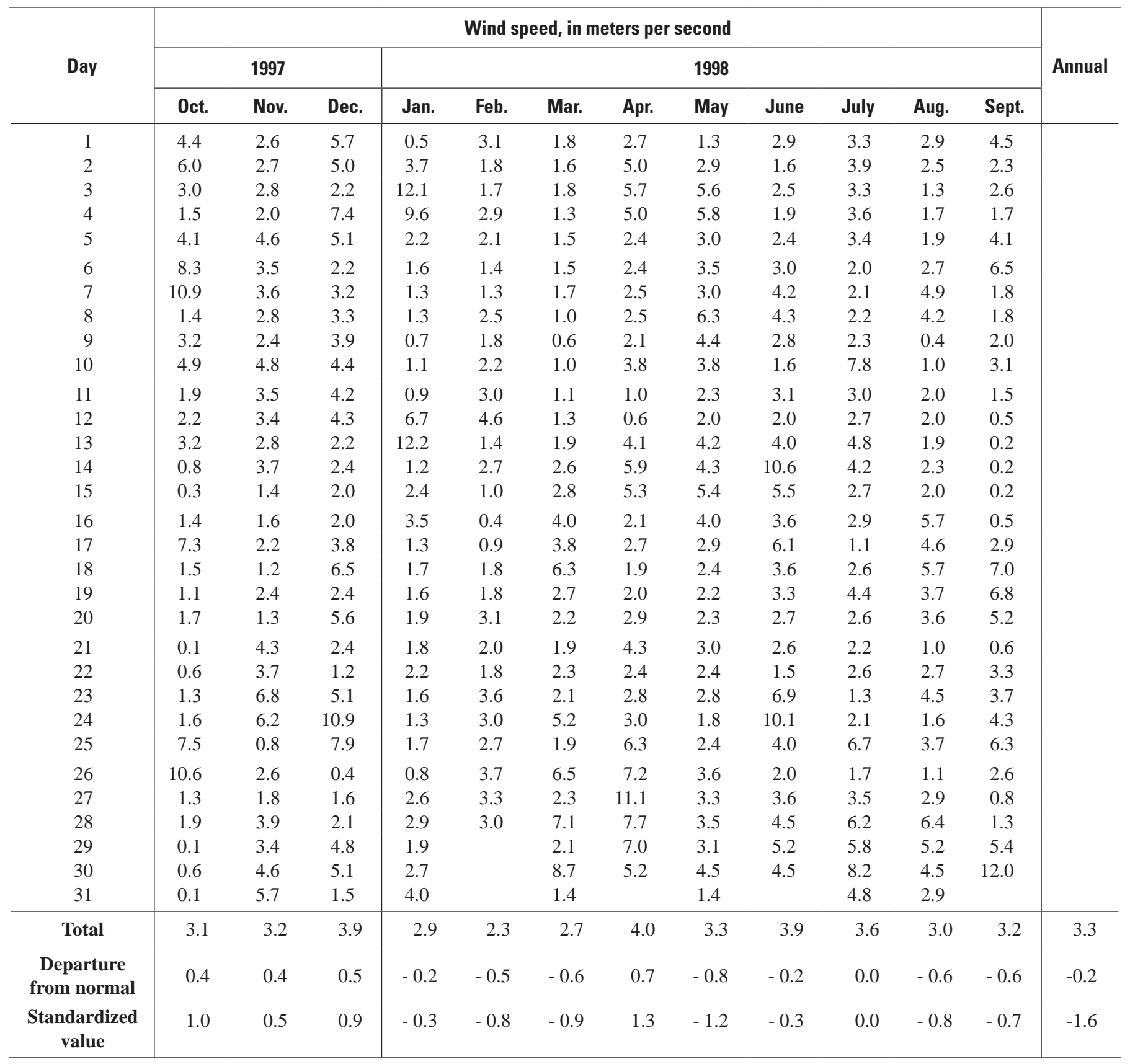


Table 26. Daily, monthly, and annual average wind speed at 1,480 meters altitude, Gulkana Glacier basin, 1999 hydrologic year.

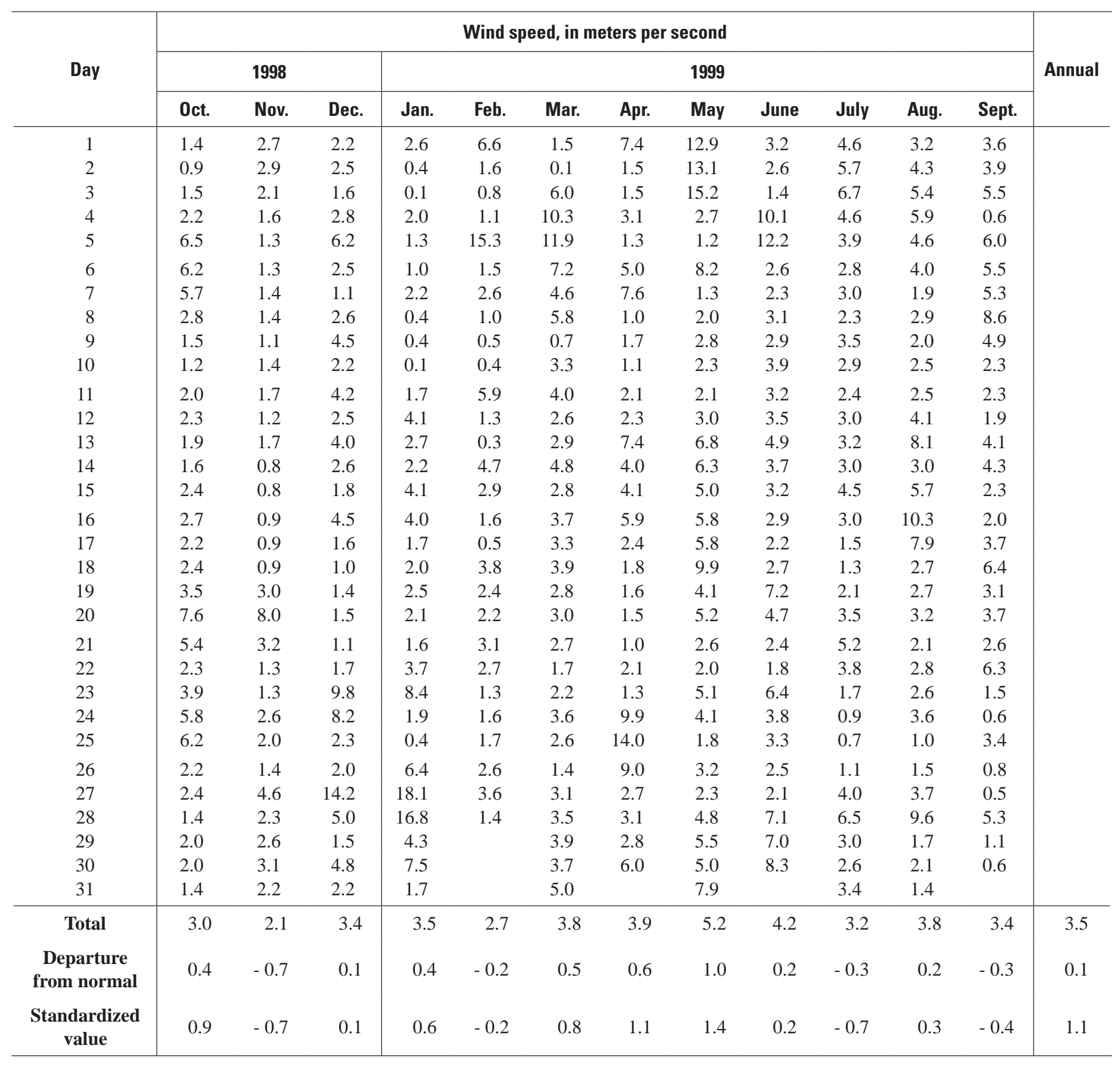


Table 27. Daily, monthly, and annual average wind speed at 1,480 meters altitude, Gulkana Glacier basin, 2000 hydrologic year.

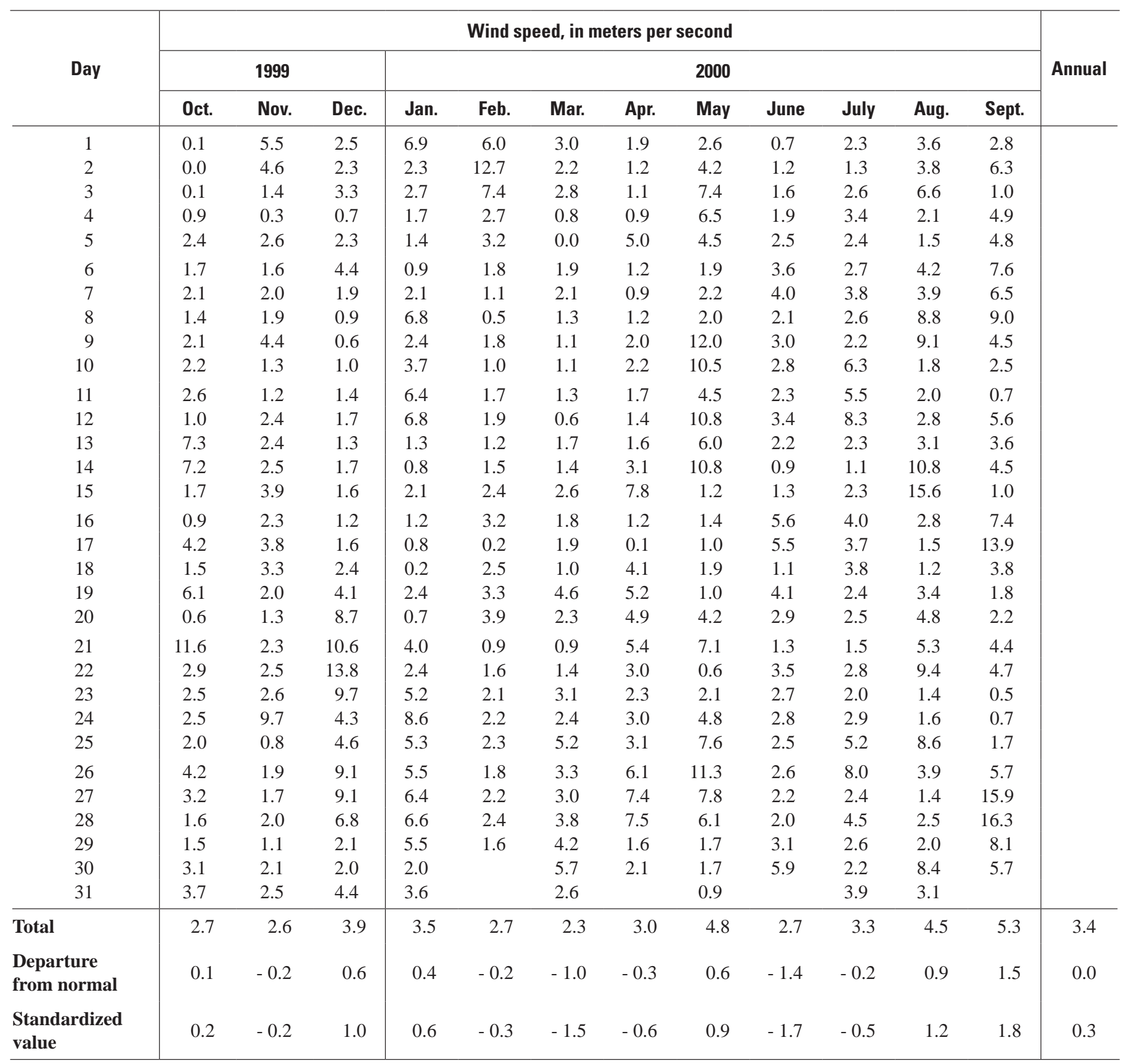


Table 28. Daily, monthly, and annual average wind speed at 1,480 meters altitude, Gulkana Glacier basin, 2001 hydrologic year. [-, daily missing value]

\begin{tabular}{|c|c|c|c|c|c|c|c|c|c|c|c|c|c|}
\hline Day & \multicolumn{12}{|c|}{ Wind speed, in meters per second } & Annual \\
\hline 1 & 3.8 & 0.6 & 2.6 & 2.4 & 4.9 & 3.2 & 2.8 & 6.5 & 12.1 & 2.7 & 5.6 & 10.0 & \\
\hline 2 & 2.6 & 1.6 & 1.6 & 2.3 & 2.1 & 2.0 & 2.2 & 5.8 & 6.9 & 3.4 & 2.7 & 2.7 & \\
\hline 3 & 1.8 & 9.1 & 4.0 & 5.6 & 1.0 & 1.9 & 7.6 & 4.7 & 1.8 & 2.4 & 3.9 & 0.8 & \\
\hline 5 & .9 & 1.6 & 5.4 & .7 & .0 & 2.8 & 5.2 & 2.9 & 1.8 & 2.2 & 3.0 & 2.4 & \\
\hline 6 & 3.0 & 1.7 & 3.3 & 3.3 & .0 & 3.4 & 2.9 & 3.6 & 5.2 & 10.1 & 2.9 & 1.8 & \\
\hline 7 & 5.6 & 1.3 & 1.6 & 4.3 & 8.8 & 3.0 & 1.4 & 6.6 & .9 & 7.2 & 1.9 & 6.7 & \\
\hline 8 & 3.3 & 1.7 & 1.4 & 5.6 & 3.1 & 2.1 & 1.7 & 1.3 & 3.7 & 1.7 & 2.2 & 2.7 & \\
\hline 9 & 3.1 & 1.8 & 2.3 & 4.2 & 6.9 & .3 & 2.4 & 1.9 & 4.1 & 2.7 & 2.0 & 2.8 & \\
\hline 10 & 1.6 & 7.4 & 4.6 & 3.6 & 1.8 & 1.9 & 3.2 & 2.5 & 3.5 & 2.6 & 2.0 & 2.1 & \\
\hline 14 & 2.6 & 3.2 & 1.8 & 4.7 & 1.6 & 2.6 & 3.1 & 4.9 & 11.9 & 5.1 & 4.1 & 2.6 & \\
\hline 15 & 1.6 & 1.1 & 1.5 & 10.3 & 3.2 & 2.4 & 5.2 & 2.5 & 12.1 & 3.1 & 1.5 & 2.4 & \\
\hline 16 & 2.7 & 2.8 & 1.9 & 4.7 & 4.1 & 3.7 & 2.3 & 1.3 & 4.2 & 4.6 & 2.5 & 2.7 & \\
\hline 17 & 2.3 & 5.2 & 2.3 & 3.1 & 3.0 & 11.3 & 2.9 & .9 & 3.3 & 3.9 & 2.6 & 4.8 & \\
\hline 18 & 2.2 & 5.2 & 2.0 & 5.1 & 2.4 & 9.6 & 2.9 & 3.8 & 2.5 & 2.7 & 2.2 & 4.9 & \\
\hline 19 & 1.1 & 4.2 & 3.7 & 6.2 & 1.7 & 8.9 & 2.0 & 1.4 & 3.0 & 3.8 & 2.9 & .9 & \\
\hline 20 & 3.3 & 1.8 & 5.0 & 2.8 & 1.3 & 2.2 & 1.6 & 2.7 & 3.8 & 3.9 & 6.3 & .7 & \\
\hline 21 & 2.6 & 4.1 & 5.3 & 3.2 & 2.0 & 3.2 & 2.0 & 1.7 & 3.6 & 3.5 & 8.1 & 1.5 & \\
\hline 22 & 1.8 & 2.6 & 4.7 & 2.4 & 1.2 & 2.5 & 3.2 & 2.0 & 6.0 & 2.7 & 8.2 & 3.0 & \\
\hline 23 & 1.0 & 1.5 & 1.9 & 5.4 & 2.8 & 1.1 & 2.7 & 2.4 & 4.3 & 1.4 & 2.3 & 6.6 & \\
\hline 24 & 2.4 & 3.3 & 2.3 & 5.1 & 4.3 & 1.2 & 6.6 & .9 & 3.1 & 2.4 & 1.6 & 2.4 & \\
\hline Monthly average & 2.5 & 3.0 & 3.0 & 3.9 & 3.3 & 3.5 & 3.3 & 3.7 & 5.1 & 3.2 & 3.1 & 3.5 & 3.4 \\
\hline $\begin{array}{c}\text { Departure } \\
\text { from normal }\end{array}$ & -.2 & .2 & -.4 & .8 & .4 & .2 & .0 & -.4 & 1.0 & -.4 & -.7 & -.3 & 0.0 \\
\hline $\begin{array}{c}\text { Standardized } \\
\text { value }\end{array}$ & -.4 & .2 & -.6 & 1.3 & .5 & .3 & -.1 & -.6 & 1.3 & -.8 & -1.1 & -.3 & 0.1 \\
\hline
\end{tabular}




\section{Streamflow}

The Phelan Creek stream-gaging station (USGS station number 15478040) is part of a large-scale USGS streammonitoring network in Alaska. Data collection and analysis are conducted by standard techniques developed by the USGS (Wahl and others, 1995). Daily values of discharge are reported along with those for the rest of Alaska in the annual USGS Water-Data Report series (U.S. Geological Survey, 1968-2002; also available at http://ak.water.usgs.gov/ glaciology/gulkana/streamflow/). An 11-year gap in the record exists from 1979-1989.
The gaging station is located at an altitude of $1,125 \mathrm{~m}$ and about $1 \mathrm{~km}$ downstream from the present glacier terminus (igs. 2, $\underline{3}$, and 15). The creekbed consists of poorly sorted gravel and small boulders, and the channel is subject to frequent changes during high flows, making it difficult to monitor accurately. Phelan Creek freezes from early autumn until mid-spring, and runoff must be estimated during this interval. Stream stage is continuously recorded, and discharge measurements are made periodically to determine the relation between stage and discharge at different streamflow levels (including ice covered) and to detect any shifts in the relation due to changes in the creekbed.

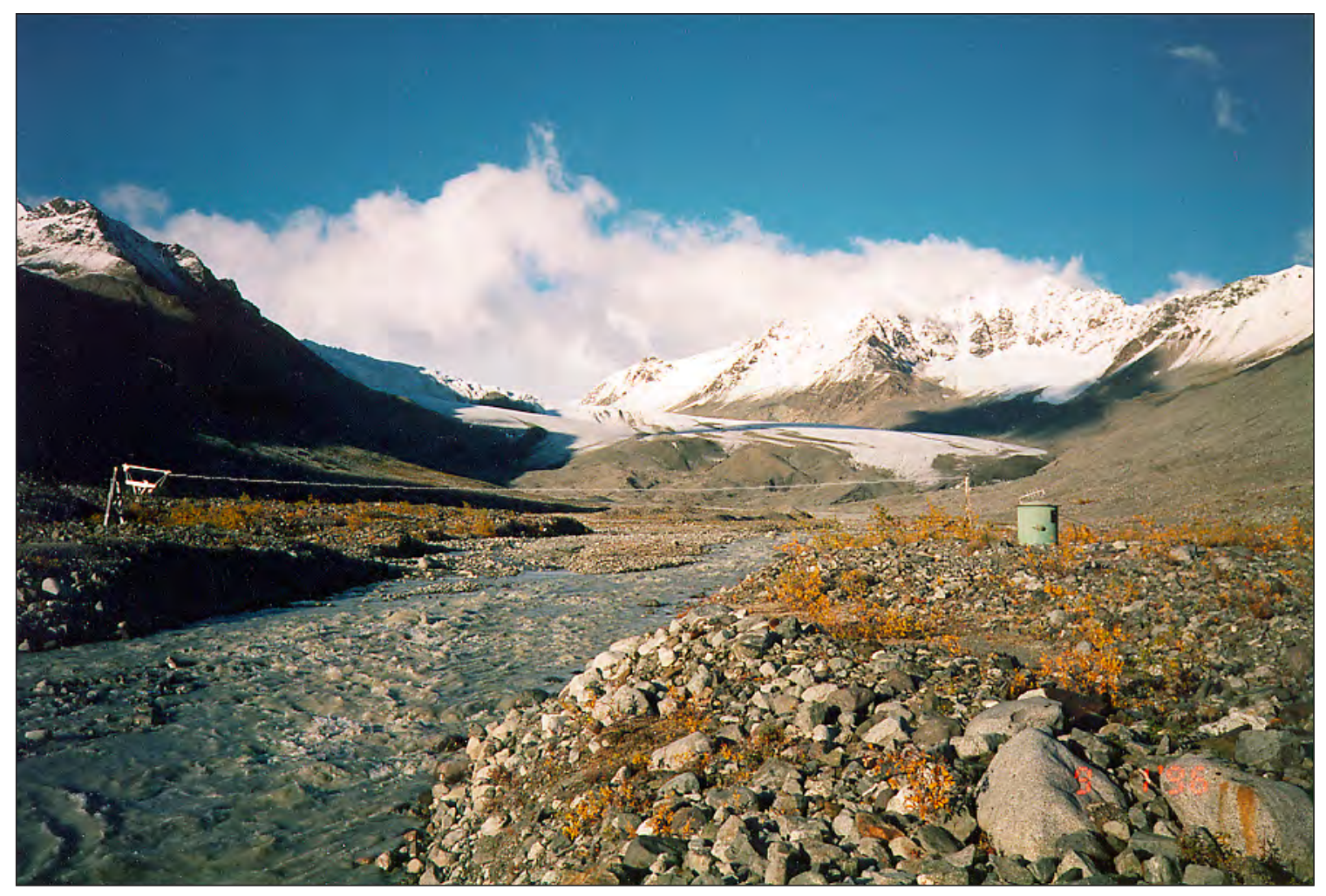

Figure 15. The Gulkana Glacier terminus from just downstream of the Phelan Creek stream-gaging station, USGS station 15478040, Alaska, September 1, 1996. The gaging station site is about $1 \mathrm{~km}$ downstream of the present glacier terminus. The channel bed is composed of typical ground moraine material, poorly sorted gravel and small boulders, which is subject to frequent changes during high flows. The mean discharge for September 1, 1996, was about 9 cubic meters per second (runoff of about 24 millimeters), a value typical of mid-summer, but high for early September. The cable way and small hand car are used for making discharge measurements when the flow is too deep and fast for safe wading. The round structure to the right of the creek houses instrumentation and satellite telemetry. 


\section{Results}

\section{Glacier Mass Balance}

Winter balance is generally less variable than summer or net balance (standard deviation of the former is about half the latter). The average measured winter balance for 1997-2001 of 1.15 mweq was about equal to the period-of-record average, though 2000 and 2001 were well above average. A linear trend in the 1966-2001 winter balance indicates an increase of $0.03 \mathrm{mweq} / \mathrm{decade}$, which is small and may be insignificant. In most years, the glacier-wide maximum snow balance occurs 4-6 weeks after the measured winter snow balance and generally after the snow balance has peaked and started to decline on the lower glacier. A simple temperature- and precipitation-forced model used to evaluate the difference between measured balance and maximum balance shows that maximum balances are generally 5-15 percent greater than the measured balance.

Net balance values are listed in table 29. The glacieraveraged, net balance year typically starts and ends about the end of August, but can start as early as mid-August or as late as the end of September. Of note, 1997 was the most negative year of record so far with a net balance of -1.71 mweq and the 1997-2001 average net balance of -0.77 mweq was about 2.5 times more negative than the previous 31-year average of -0.30 mweq (fig. 16). The 1966-2001 linear regression trend shows a decreasing net balance by 0.20 mweq/decade. Summer balance shows large changes over the project history. The year 1997 also exhibited the most negative summer balance of -2.71 mweq and furthermore the 1997-2001 average summer balance of -1.92 mweq was almost 1 standard deviation below the previous 31-year average of -1.41 mweq. The 1966-2001 linear regression trend shows a decreasing summer balance by 0.18 mweq/decade.

As previously discussed, the integral in equation (5) was estimated as the sum of the product of site balances $b_{A}, b_{B}$, and $b_{D}$ (fig. 2), and area weighting coefficients which were determined from maps. As examples, for 1966 the estimation is:

$$
\bar{b}=0.203 b_{A}+0.270 b_{B}+0.527 b_{D}+b_{\text {int }},
$$

and for 2001:

$$
\bar{b}=0.157 b_{A}+0.198 b_{B}+0.645 b_{D}+\bar{b}_{\text {int }} .
$$

These examples show decreased representation of the region represented by site A as the lower glacier thins and retreats. During the same time interval, little change occurred at upper site $\mathrm{D}$, which represents more of the glacier area.

Table 30 lists detailed glacier-wide, reference surface balances (Elsberg and others, 2001, Harrison and others, 2005) for the 5 years of this study and table 31 provides a summary for all years. Reference surface balances are calculated by the same method as the conventional balances except that the AAD is fixed in 1966 at the beginning of the record. Reference surface balances correct the balances by removing the glacier's dynamic response of the glacier from the balance as if its area or altitude had remained invariant. Some find this balance parameter is better suited for climate analysis in longterm studies than the conventional balance. For instance, when climate undergoes a permanent step change, the conventional balance of a glacier initially in equilibrium with the climate undergoes only a temporary step change then slowly reverts back to its equilibrium zero balance, but the reference surface balance, like the climate, will undergo a permanent step change. The correction is large for Gulkana Glacier as shown in figure 17. The cumulative reference surface balance volume for the 36-year period of record is 28 percent more negative than the conventional balance volume.

Estimates of the ELA and AAR are listed in table 29. The 1997-2001 average AAR is about 0.53 compared to the previous 31 -year average of 0.60 , which represents approximately a 35-m rise in the ELA. Both parameter changes are consistent with the trend towards more negative balances. 
Table 29. Index-site and area-integrated conventional balance quantities for Gulkana Glacier and Gulkana Glacier basin, 1997-2001 hydrologic years.

[Abbreviations: m, meters; mweq, meters water equivalent; mm-dd-yy, month-day-year; $\mathrm{km}^{2}$, square kilometers; ${ }^{\circ} \mathrm{C}$, degrees Celsius]

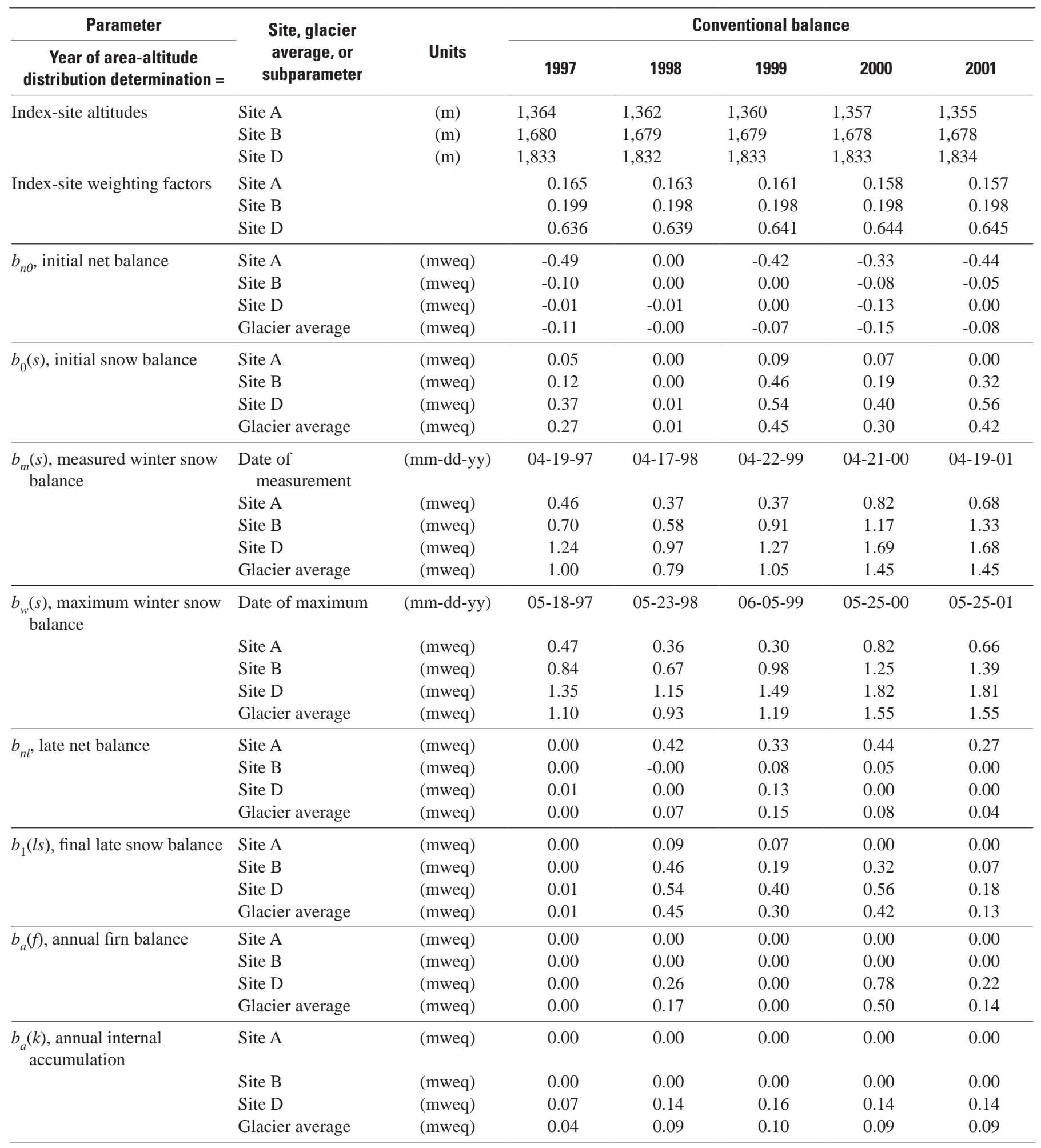


Table 29. Index-site and area-integrated conventional balance quantities for Gulkana Glacier and Gulkana Glacier basin, 1997-2001 hydrologic years.-Continued

[Abbreviations: m, meters; mweq, meters water equivalent; mm-dd-yy, month-day-year; km² , square kilometers; ${ }^{\circ} \mathrm{C}$, degrees Celsius]

\begin{tabular}{|c|c|c|c|c|c|c|c|}
\hline $\begin{array}{c}\text { Parameter } \\
\text { Year of area-altitude } \\
\text { distribution determination = }\end{array}$ & $\begin{array}{l}\text { Site, glacier } \\
\text { average, or } \\
\text { subparameter }\end{array}$ & Units & \multicolumn{5}{|c|}{ Conventional balance } \\
\hline \multirow{2}{*}{$\begin{array}{l}b_{a}(i), \text { annual old firn and ice } \\
\text { balance }\end{array}$} & Site B & (mweq) & -2.30 & -0.95 & -1.35 & -0.14 & -0.99 \\
\hline & Site D & (mweq) & -0.49 & 0.00 & -0.27 & 0.00 & 0.00 \\
\hline \multirow[t]{4}{*}{$\begin{array}{l}b_{a}(j), \text { annual internal ablation } \\
\quad \text { (glacier averaged) }\end{array}$} & $\begin{array}{l}\text { From geothermal } \\
\text { heat flux }\end{array}$ & (mweq) & -0.005 & -0.005 & -0.005 & -0.005 & -0.005 \\
\hline & $\begin{array}{l}\text { From potential } \\
\text { energy loss from } \\
\text { ice motion }\end{array}$ & (mweq) & -0.005 & -0.005 & -0.005 & -0.005 & -0.005 \\
\hline & $\begin{array}{l}\text { From potential } \\
\text { energy loss } \\
\text { from water flow } \\
\text { (estimated) }\end{array}$ & (mweq) & -0.075 & -0.060 & -0.054 & -0.035 & -0.048 \\
\hline & Total & (mweq) & -0.08 & -0.07 & -0.06 & -0.04 & -0.06 \\
\hline \multirow[t]{6}{*}{$b_{n}$, net balance } & $\begin{array}{l}\text { Start of net balance } \\
\text { year for glacier } \\
\text { average }\end{array}$ & (mm-dd-yy) & $08-25-96$ & 09-28-97 & 08-21-98 & 09-03-99 & $08-18-00$ \\
\hline & $\begin{array}{l}\text { End of net balance } \\
\text { year for glacier } \\
\text { average }\end{array}$ & (mm-dd-yy) & $09-27-97$ & 08-20-98 & 09-02-99 & $08-17-00$ & 09-03-01 \\
\hline & Site A & (mweq) & -5.34 & -3.07 & -4.44 & -2.41 & -4.13 \\
\hline & Site B & (mweq) & -2.39 & -0.95 & -1.27 & -0.17 & -1.04 \\
\hline & Site D & (mweq) & -0.43 & 0.39 & 0.02 & 0.79 & 0.36 \\
\hline & $\begin{array}{l}\text { Glacier average } \\
\left.\quad \text { (includes } b_{\mathrm{a}}(j)\right)\end{array}$ & (mweq) & -1.71 & -0.51 & -1.02 & 0.06 & -0.68 \\
\hline \multicolumn{2}{|l|}{ ELA, equilibrium-line altitude } & $(\mathrm{m})$ & 1,867 & 1,787 & 1,831 & 1,705 & 1,794 \\
\hline \multicolumn{2}{|l|}{ Accumulation area } & $\left(\mathrm{km}^{2}\right)$ & 8.08 & 10.07 & 8.83 & 11.41 & 9.51 \\
\hline \multicolumn{2}{|l|}{ Ablation area } & $\left(\mathrm{km}^{2}\right)$ & 10.04 & 8.06 & 9.29 & 6.72 & 8.61 \\
\hline \multicolumn{2}{|l|}{ AAR, accumulation-area ratio } & & 0.45 & 0.56 & 0.49 & 0.63 & 0.52 \\
\hline \multicolumn{2}{|c|}{ Calculated annual precipitation Basin average } & $(\mathrm{m})$ & 1.34 & 1.98 & 1.22 & 1.66 & 1.37 \\
\hline Annual basin runoff & $\begin{array}{l}\text { 1,125-meter stream } \\
\text { gage }\end{array}$ & (m) & 2.54 & 2.34 & 1.93 & 1.62 & 1.85 \\
\hline Percent of runoff from balance & Basin value & (percent) & 47 & 15 & 37 & -2 & 26 \\
\hline
\end{tabular}



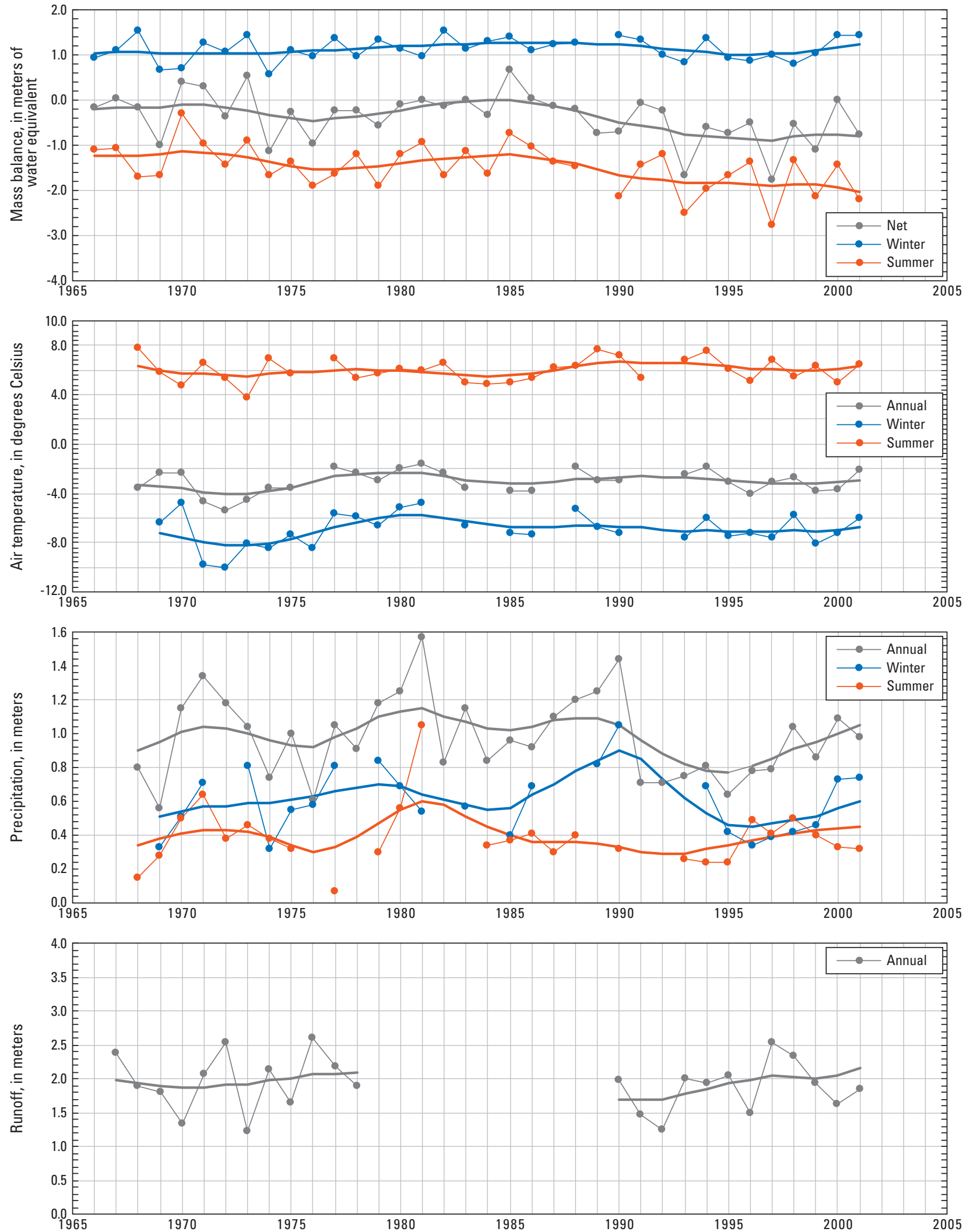

Figure 16. Net, winter, and summer values for mass balance, air temperature, precipitation catch, and runoff, Gulkana Glacier basin, Alaska, water years 1966-2001. 
Table 30. Index-site and area-integrated reference surface balance quantities for Gulkana Glacier and Gulkana Glacier Basin, 1997-2001 hydrologic years.

[Reference surface is 1967 area-altitude distribution. Abbreviations: m, meters; mweq, meters water equivalent; mm-dd-yy, month-day-year; km², square kilometers; ${ }^{\circ} \mathrm{C}$, degrees Celsius]

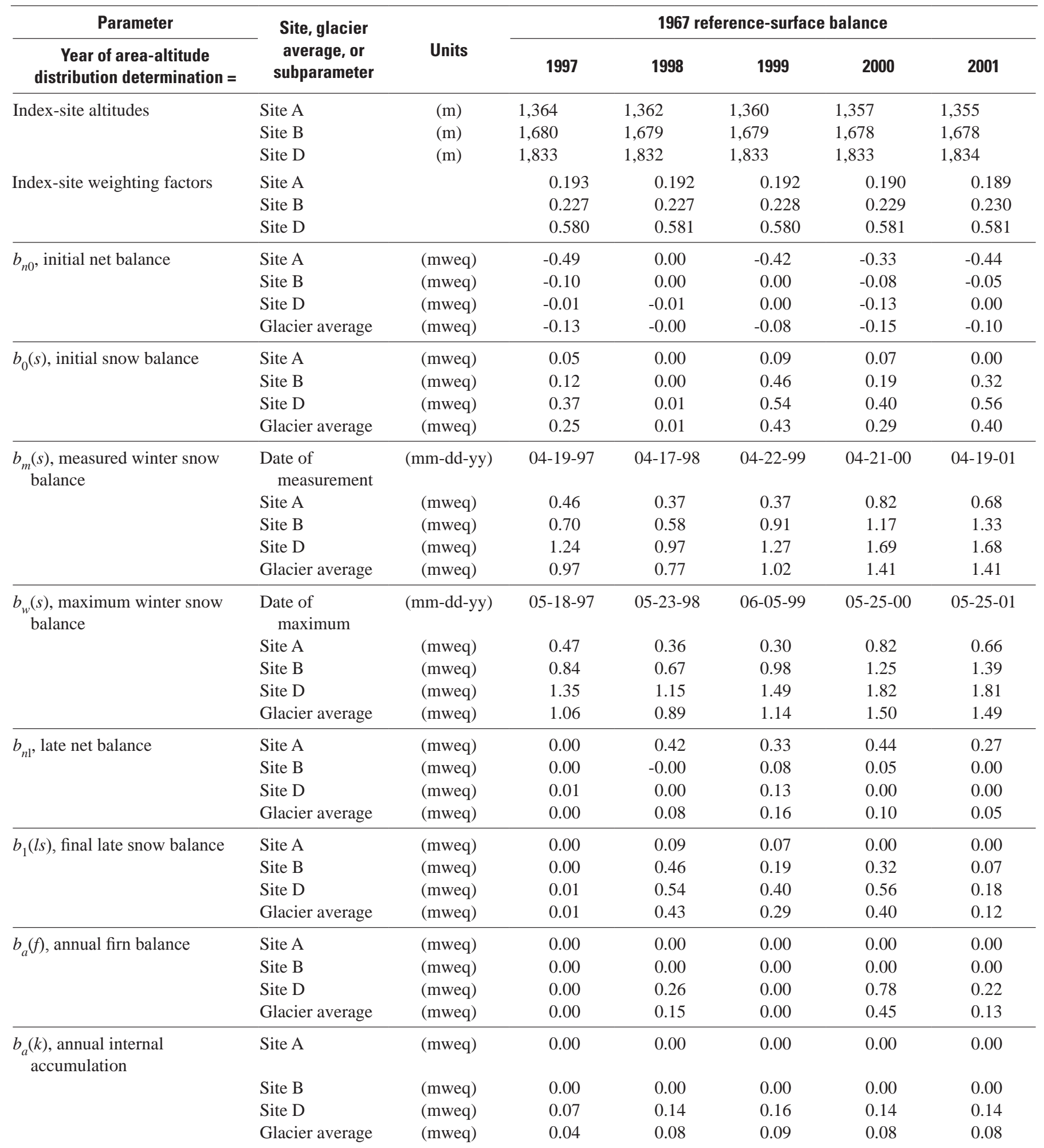


Table 30. Index-site and area-integrated reference surface balance quantities for Gulkana Glacier and Gulkana Glacier Basin, 1997-2001 hydrologic years. - Continued

[Reference surface is 1967 area-altitude distribution. Abbreviations: m, meters; mweq, meters water equivalent; mm-dd-yy, month-day-year; km², square kilometers; ${ }^{\circ} \mathrm{C}$, degrees Celsius]

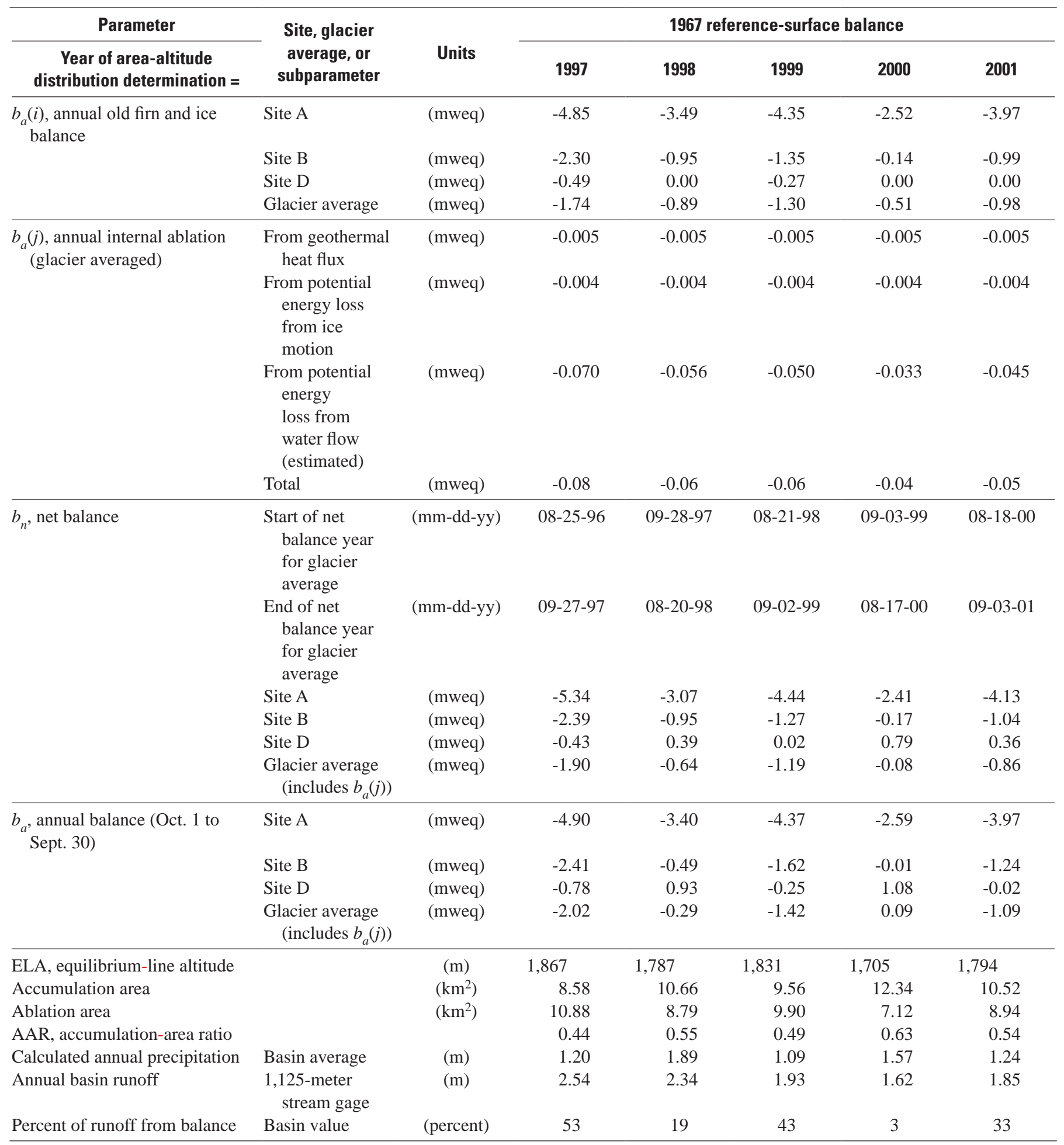


Table 31. Conventional and reference surface yearly and cumulative net balances and cumulative volume changes.

[Abbreviations: mweq, meters water equivalent; $\mathrm{km}^{3}$ weq, cubic kilometers water equivalent]

\begin{tabular}{|c|c|c|c|c|c|c|}
\hline \multirow[b]{2}{*}{ Year } & \multicolumn{3}{|c|}{ Conventional-net balance } & \multicolumn{3}{|c|}{ Reference-surface net balance } \\
\hline & $\begin{array}{l}\text { Yearly } \\
\text { (mweq) }\end{array}$ & $\begin{array}{l}\text { Cumulative } \\
\text { (mweq) }\end{array}$ & $\begin{array}{c}\text { Cumulative } \\
\text { volume } \\
\text { (km³ weq) }\end{array}$ & $\begin{array}{c}\text { Yearly } \\
\text { (mweq) }\end{array}$ & $\begin{array}{l}\text { Cumulative } \\
\text { (mweq) }\end{array}$ & $\begin{array}{c}\text { Cumulative } \\
\text { volume } \\
\left(\mathrm{km}^{3} \text { weq }\right)\end{array}$ \\
\hline 1966 & -0.16 & -0.16 & -0.003 & -0.16 & -0.16 & -0.003 \\
\hline 1967 & 0.02 & -0.14 & -0.003 & 0.01 & -0.15 & -0.003 \\
\hline 1968 & -0.17 & -0.31 & -0.006 & -0.18 & -0.34 & -0.007 \\
\hline 1969 & -1.00 & -1.31 & -0.025 & -1.03 & -1.37 & -0.027 \\
\hline 1970 & 0.39 & -0.92 & -0.018 & 0.34 & -1.03 & -0.020 \\
\hline 1971 & 0.29 & -0.63 & -0.012 & 0.24 & -0.79 & -0.015 \\
\hline 1972 & -0.37 & -1.00 & -0.019 & -0.42 & -1.22 & -0.024 \\
\hline 1973 & 0.54 & -0.46 & -0.009 & 0.46 & -0.75 & -0.015 \\
\hline 1974 & -1.12 & -1.58 & -0.030 & -1.21 & -1.96 & -0.038 \\
\hline 1975 & -0.25 & -1.83 & -0.034 & -0.34 & -2.30 & -0.045 \\
\hline 1976 & -0.96 & -2.79 & -0.052 & -1.05 & -3.35 & -0.065 \\
\hline 1977 & -0.24 & -3.03 & -0.056 & -0.36 & -3.71 & -0.072 \\
\hline 1978 & -0.22 & -3.25 & -0.060 & -0.33 & -4.04 & -0.079 \\
\hline 1979 & -0.56 & -3.81 & -0.071 & -0.67 & -4.71 & -0.092 \\
\hline 1980 & -0.09 & -3.90 & -0.072 & -0.20 & -4.92 & -0.096 \\
\hline 1981 & 0.02 & -3.89 & -0.072 & -0.07 & -4.99 & -0.097 \\
\hline 1982 & -0.14 & -4.03 & -0.075 & -0.26 & -5.25 & -0.102 \\
\hline 1983 & 0.00 & -4.03 & -0.074 & -0.15 & -5.39 & -0.105 \\
\hline 1984 & -0.34 & -4.36 & -0.081 & -0.45 & -5.84 & -0.114 \\
\hline 1985 & 0.66 & -3.70 & -0.069 & 0.55 & -5.29 & -0.103 \\
\hline 1986 & 0.04 & -3.65 & -0.068 & -0.07 & -5.36 & -0.104 \\
\hline 1987 & -0.14 & -3.79 & -0.070 & -0.26 & -5.62 & -0.109 \\
\hline 1988 & -0.20 & -3.99 & -0.074 & -0.34 & -5.96 & -0.116 \\
\hline 1989 & -0.73 & -4.73 & -0.087 & -0.88 & -6.85 & -0.133 \\
\hline 1990 & -0.71 & -5.44 & -0.100 & -0.83 & -7.68 & -0.149 \\
\hline 1991 & -0.08 & -5.52 & -0.102 & -0.20 & -7.88 & -0.153 \\
\hline 1992 & -0.23 & -5.74 & -0.106 & -0.33 & -8.21 & -0.160 \\
\hline 1993 & -1.68 & -7.42 & -0.136 & -1.82 & -10.03 & -0.195 \\
\hline 1994 & -0.58 & -8.00 & -0.147 & -0.72 & -10.75 & -0.209 \\
\hline 1995 & -0.70 & -8.70 & -0.159 & -0.83 & -11.58 & -0.225 \\
\hline 1996 & -0.46 & -9.17 & -0.168 & -0.62 & -12.20 & -0.237 \\
\hline 1997 & -1.71 & -10.88 & -0.199 & -1.90 & -14.10 & -0.274 \\
\hline 1998 & -0.51 & -11.38 & -0.208 & -0.64 & -14.74 & -0.287 \\
\hline 1999 & -1.02 & -12.40 & -0.226 & -1.19 & -15.93 & -0.310 \\
\hline 2000 & 0.06 & -12.35 & -0.225 & -0.08 & -16.01 & -0.311 \\
\hline 2001 & -0.68 & -13.03 & -0.238 & -0.86 & -16.87 & -0.328 \\
\hline
\end{tabular}




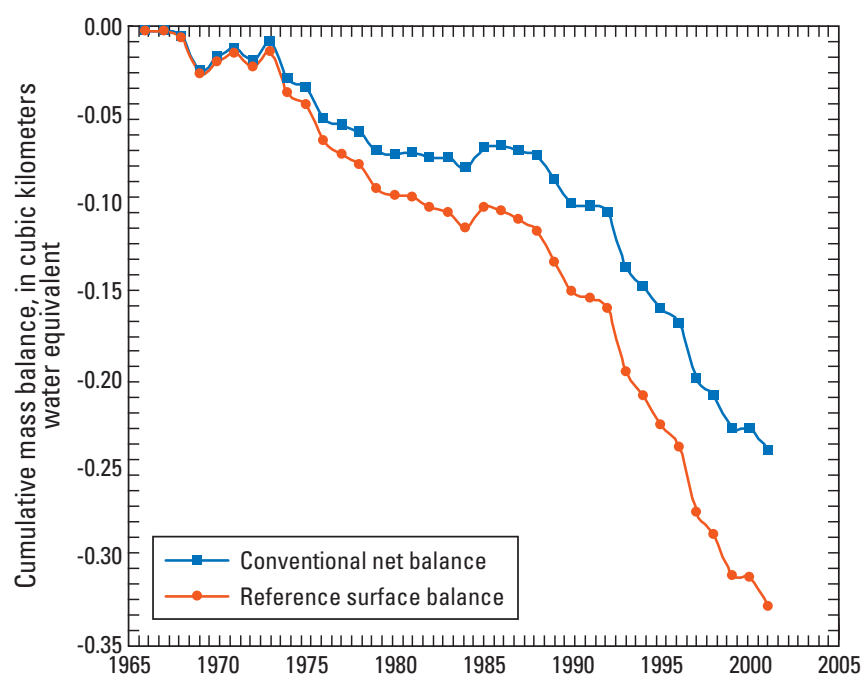

Figure 17. Cumulative conventional net balance and the cumulative reference surface net balance using the reference surface area altitude distribution of 1967, Gulkana Glacier, Alaska. Cumulative reference surface net balance volume is 38 percent more negative than the cumulative conventional net balance volume at the end of this 36 -year period.

\section{Glacier Geometry}

Measurements of glacier geometry include AADs, terminus position, and glacier surface altitude. Selected AADs are shown in figure 4 and all are given in table 2. Between the LIA maximum and 2001, the area of Gulkana Glacier decreased by $8.2 \mathrm{~km}^{2}$ or 33 percent. Between 1966 and 2001, the area decreased by $2.8 \mathrm{~km}^{2}$ or 14 percent.

Our research shows that the terminus retreated 1,650 m between 1949 (U.S. Geological Survey 1:63,360-scale Mt. Hayes A-3 quadrangle topographic map) and 1996 at an average retreat rate of $35 \mathrm{~m} / \mathrm{yr}$. Since 1996, retreat rates have been 15-20 m/yr along the south margin where the terminus is fed by the main trunk of the glacier. Along the southwest margin, an area not fed directly by the main trunk, retreat is two to three times faster than along the south margin. Faster retreat is expected to continue along the southwest margin as source ice for this area gets cutoff by the thinning of ice coming over the icefall just upstream.

We show the measured glacier surface altitude in figure 6 and table 10. Between 1997 and 2001, the general thinning trend of the 1990s continued. However, between 2000-02, the glacier began to thicken at site $\mathrm{D}$, reversing the thinning of the previous 10-15 years.

\section{Ice Motion}

At site $\mathrm{A}$ in the ablation zone, we measured speeds between 10-15 m/yr (table 11); higher speeds between 30-40 m/yr occur at site B near the ELA (table 12); motion at site $\mathrm{D}$ in the lower accumulation zone ranges between 35-50 m/yr (table 13). Motion varies seasonally with summer speeds 20-30 percent greater than winter speeds. A general slowing trend began in the early 1990s with speeds reduced by almost 40 percent since then. As stated in the methods, stake positions are known to the highest level of accuracy possible, given the leaning and bending of stakes after installation. Taken together, all sources of error yield position estimates accurate to $0.3 \mathrm{~m}$, resulting in well-resolved speed estimates over seasonal and annual time scales.

\section{Meteorology}

During 1997-2001, winter, summer, and annual average air temperatures (fig. 16) were all about normal for the 19682001 period of record $\left(-7^{\circ} \mathrm{C},+6^{\circ} \mathrm{C}\right.$, and $-3^{\circ} \mathrm{C}$ respectively). Daily and monthly values with statistics are shown in figures 8-12 and tables 14-18. Daily mean temperatures reach $+15^{\circ} \mathrm{C}$ in summer and $-30^{\circ} \mathrm{C}$ in winter. A small warming trend exists in the data; the annual trend is $+0.16^{\circ} \mathrm{C} /$ decade, the winter trend is $+0.17^{\circ} \mathrm{C} /$ decade, and the summer trend is $+0.10^{\circ} \mathrm{C} /$ decade. We compared temperature data over the 3 -year interval of different instrument overlap, which indicates that the digital sensors average $1.0^{\circ} \mathrm{C}$ warmer than the analog sensor (igg. 13, tables 14-15). The difference exhibits both seasonal and interannual variability, but the variability from the average of $+1.0^{\circ} \mathrm{C}$ is within the accuracy threshold of the analog sensor. To remedy the sensor differences or bias, $1.0^{\circ} \mathrm{C}$ was added to all values in the analog record (October 1, 1967September 30, 1998).

Over the 5 years of our study, annual average wind speed was $3.5 \mathrm{~m} / \mathrm{s}$. Daily and monthly average and period-ofrecord average monthly wind speeds are shown in figure 14 and tables 24-28. Seasonal variability was small, and winds were slightly stronger in summer. Maximum daily average wind speed for these years was $18.1 \mathrm{~m} / \mathrm{s}$; the maximum 15 -minute average wind speed was $32.7 \mathrm{~m} / \mathrm{s}$. Wind speeds exceeded the threshold of 5-8 m/s for blowing snow (Barry, 1989) 15-20 percent of the time. Hence, wind entrainment and redeposition of snow is an important process in the mass balance of Gulkana Glacier. Wind-speed data have been collected at Gulkana Glacier only since 1996 prohibiting any analysis of trends. 
Precipitation catch (figs. 8-12 and 14, tables 19-23) averages about $1 \mathrm{~m} / \mathrm{yr}$, with monthly averages of 5-6 cm from November through June, but is two to three times greater during summer and early autumn. (Note that this apparent seasonal increase in precipitation may be negated by a seasonal change in the catch efficiency; see discussion of catch efficiency later in this section.) During this 5-year study, annual precipitation catch was about equal to the 1968-2001 average. Precipitation shows significant variability at decadal time scales (fig. 16). Linear regression trend analysis for 1968-2001 shows a decrease of about 2-3 percent per decade in the annual and summer values, but no trend in the winter values. The statistical significance of these small trends was not estimated.

Detailed knowledge of the gage efficiency is highly desired, and we have analyzed the relation between gage catch and true basin precipitation. As a starting point, we compared the Gulkana data to the nearest National Weather

Service station, in Paxson (station 50709704), $20 \mathrm{~km}$ south of the glacier at an altitude of $823 \mathrm{~m}$. Precipitation recorded at Paxson data between 1975-84 and 1986-92 is approximately 50 percent lower than at Gulkana Glacier station. Correlation between the two data sets is with only a moderate Pearson correlation coefficient of 0.62 between the two daily time series. Hence, Paxson precipitation is considered a poor proxy to Gulkana Glacier basin precipitation explaining less than 40 percent of the variability of the Gulkana record.

Gage catch efficiencies vary with wind speed, direction, and the nature of the precipitation. For an identical gage at Wolverine Glacier, which has a windier environment than Gulkana, Mayo and others (1992) determined that the gagecatch efficiency was 0.31 over a 10 -year period. Estimates for water years 1968-78 and 1990-2001 at Gulkana Glacier, yielded a long-term average catch efficiency of about 60 percent assuming precipitation variations over the basin, evaporation, condensation, and sublimation are insignificant. Despite the low catch efficiency, precipitation catch is useful as an indicator of true basin precipitation.

In 1996, a complete mockup of our gage and wind shield was sent to Environment Canada's Climate Processes Section, Climate Research Division, Science and Technology Branch, to calibrate the precipitation catch efficiency as a function of wind speed. They analyzed data from winter, 1999, studying 21 precipitation events (5 rain events, 6 mixed precipitation events, and 10 snow events) total. These storms each deposited more than $3 \mathrm{~mm}$ of precipitation and ranged in duration between 2 hours and 3 days (fig. 18). The highest average wind speeds for individual events were $6.1 \mathrm{~m} / \mathrm{s}$ for rain, $5.7 \mathrm{~m} / \mathrm{s}$ for mixed precipitation, and $4.8 \mathrm{~m} / \mathrm{s}$ for snow, considerably less than the maximum daily average local wind speeds, but consistent with monthly average wind speeds. True precipitation for the study was estimated from a gage inside a double fenced intercomparison reference shield (DFIR). The DFIR is designated as the standard reference shield by the World Meteorological Organization for collection of true precipitation (Goodison and others, 1998) and the shield is recommended for use in the U.S. Climate Reference Network. For rain events, linear regression analysis suggests the Gulkana Glacier gage collects true precipitation when wind speeds are less than $6 \mathrm{~m} / \mathrm{s}$. For mixed rain and snow events, catch efficiency drops by 3 percent for every $1 \mathrm{~m} / \mathrm{s}$ increase in wind speed, and for pure snow events, catch efficiency is reduced 10 percent for every $1 \mathrm{~m} / \mathrm{s}$ increase in wind speed. Corrections have not been applied to the data based on these findings, but the statistics reveal patterns present in the data that may not be explained by true precipitation trends. Note that our statistics are weak as only 21 storm events from one winter were analyzed.

\section{Basin Runoff}

Typical summer discharge from Phelan Creek ranges from 4 to $20 \mathrm{~m}^{3} / \mathrm{s}$, and exhibits strong diurnal fluctuations. Winter values are more constant and range from 0.04 to $0.1 \mathrm{~m}^{3} / \mathrm{s}$, which is about three to four times the average contribution from geothermal and strain heating forced melt of the glacier bed (March and Trabant, 1997). Record peak discharge is $65 \mathrm{~m}^{3} / \mathrm{s}$ (Linn and others, 1997). The 1997-2001 discharge records are described fully with the Alaska water data in the USGS Water-Data Report annual series AK-97-1 to AK-01-1 (U.S. Geological Survey, 1968-2002). The low frequency of discharge measurements coupled with the poor quality of gage-height record and the changeable nature of the creekbed result in a discharge record that was rated as poor, meaning the standard error is greater than \pm 7.5 percent of the true value (Linn and others, 1997). The standard error of the daily discharge values at Phelan Creek gaging station during summer open-water conditions previously was estimated at 10 percent, except for those periods when discharge was estimated, in which case a 20 percent standard error was assumed (R. Kemnitz, U.S. Geological Survey, oral commun., 1997). These estimates of error are considered generally applicable for the discharge record discussed in this report.

Daily mean discharge data were converted to runoff by dividing the discharge values by the basin area (figs. 8-12, tables 32-36). Runoff is estimated for the frozen period (from about October 1 to May 31) on the basis of air temperature, precipitation, and a few winter discharge measurements. During this time, runoff typically undergoes a gradual decline, referred to as the winter recession, with little interannual variability. From pre-1996 data, we estimate winter runoff standard errors to be less than $\pm 0.2 \mathrm{~mm} / \mathrm{d}$ with increasing errors up to $\pm 5 \mathrm{~mm} / \mathrm{d}$ coinciding with the onset of the melt season by early June. 

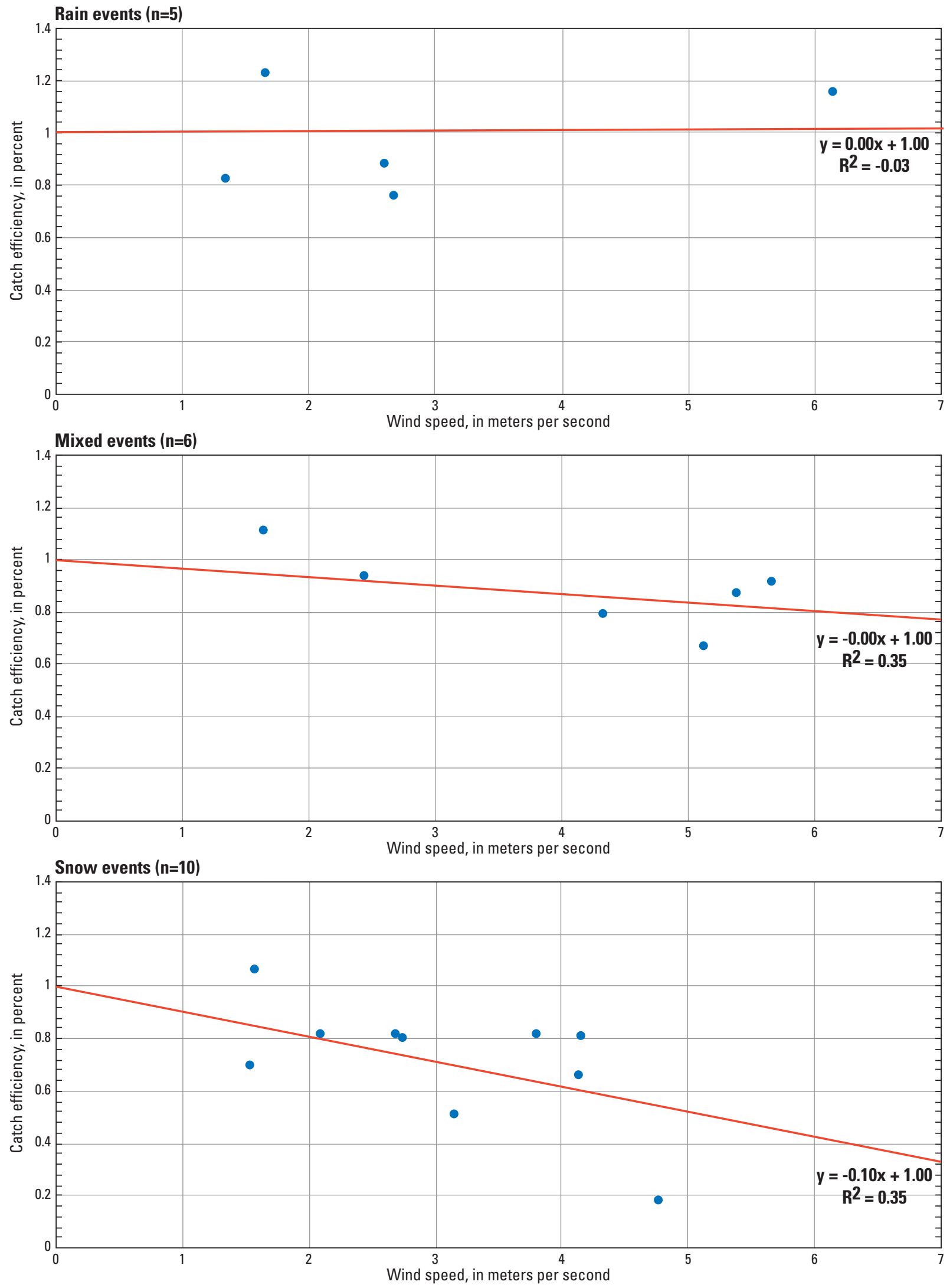

Figure 18. Precipitation gage catch efficiency relative to wind speed of the U.S. Geological Survey gage shield type in the Gulkana Glacier basin, Alaska, compared to the double fenced intercomparison reference shield for precipitation events greater than 3 millimeters during the winter 1997-98 at Toronto, Ontario. 
Table 32. Daily mean, monthly, and annual runoff from the Gulkana Glacier Basin, 1997 hydrologic year.

[Values in millimeters, averaged over the basin; except standardized values which are in units of standard deviation. Data in parentheses ( ) indicate value is estimated (see text for explanation)]

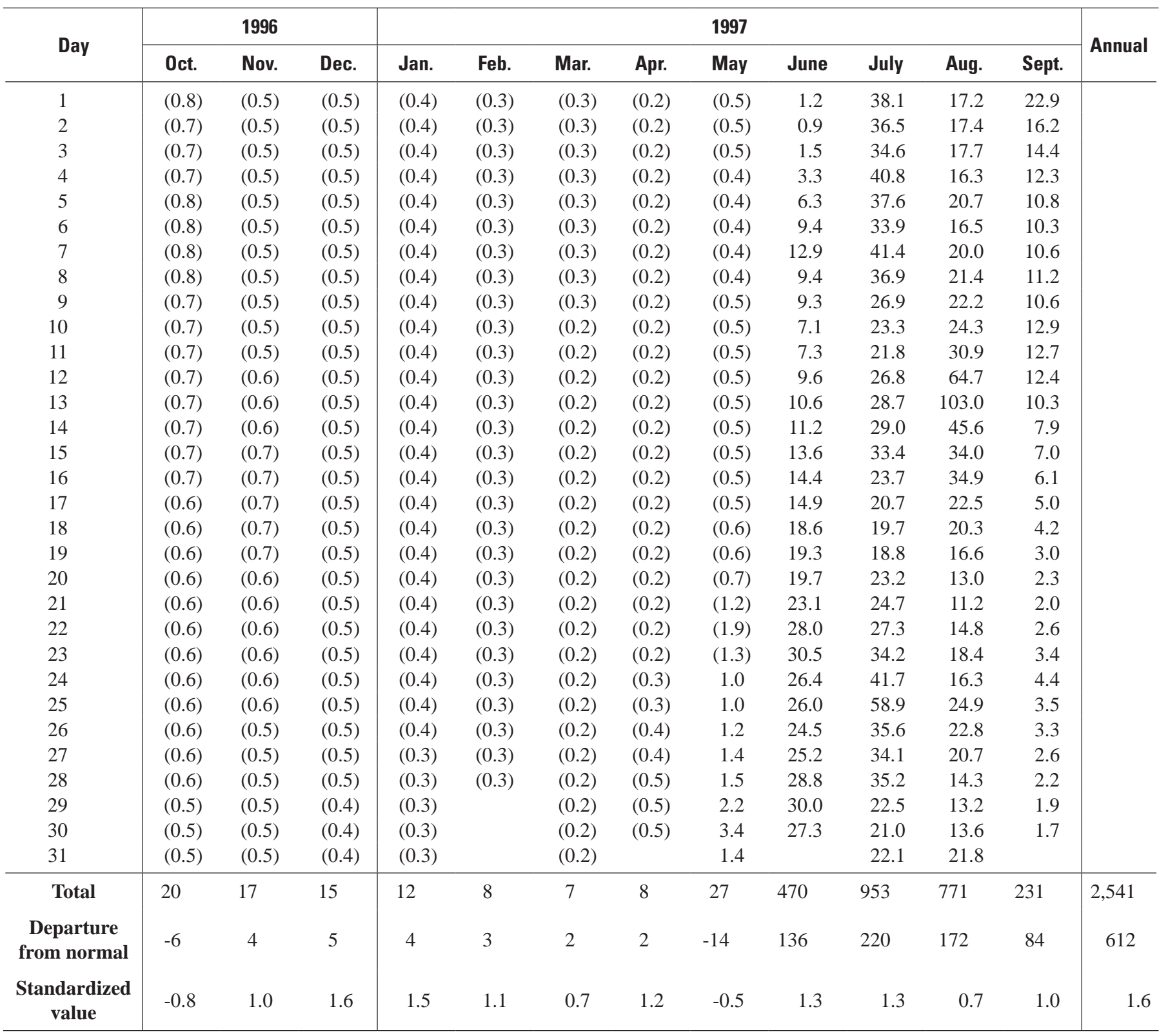


Table 33. Daily mean, monthly, and annual runoff from the Gulkana Glacier Basin, 1998 hydrologic year.

[Values in millimeters, averaged over the basin; except standardized values which are in units of standard deviation. Data in parentheses ( ) indicate value is estimated (see text for explanation)]

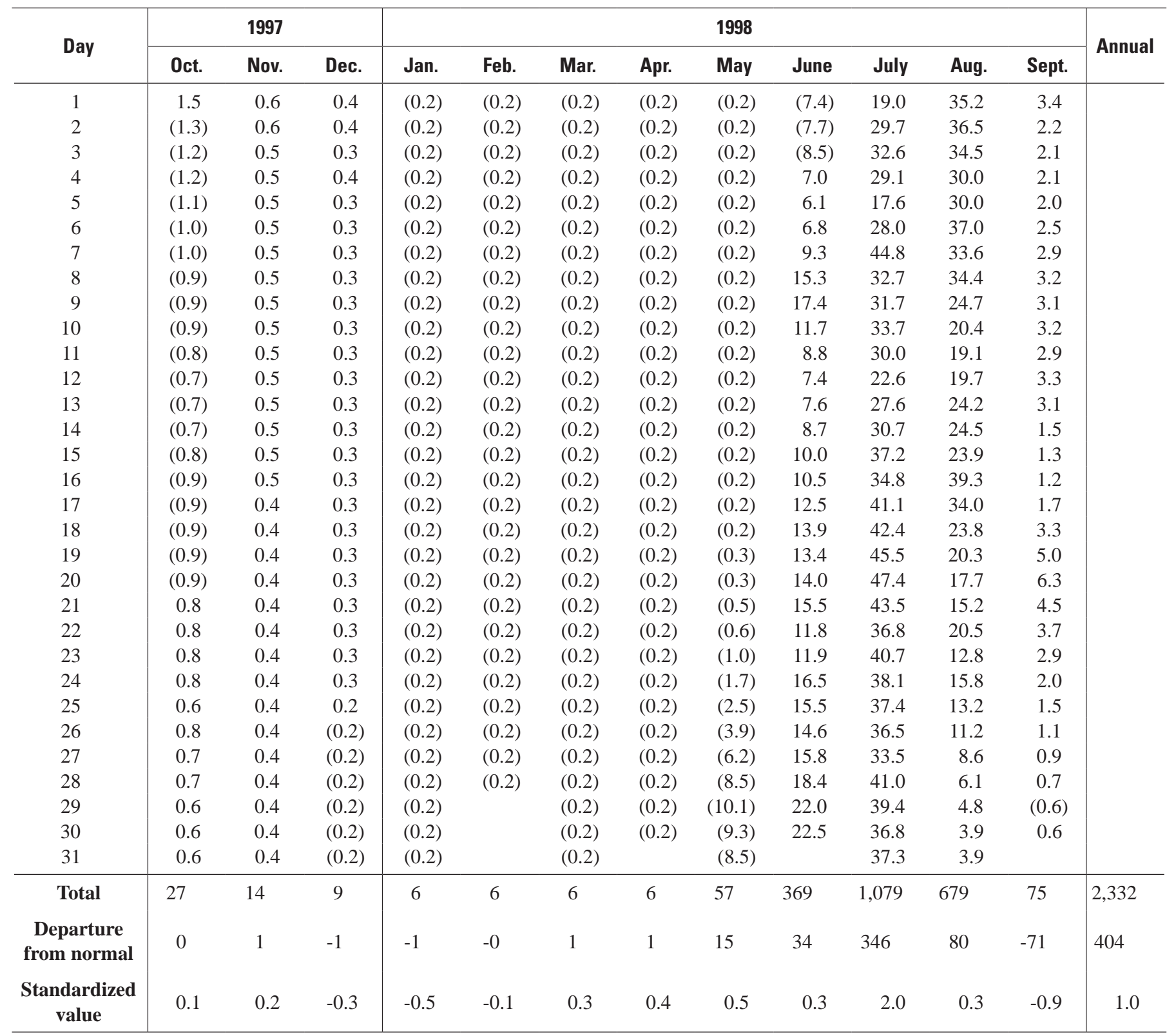


Table 34. Daily mean, monthly, and annual runoff from the Gulkana Glacier Basin, 1999 hydrologic year.

[Values in millimeters, averaged over the basin; except standardized values which are in units of standard deviation. Data in parentheses ( ) indicate value is estimated (see text for explanation)]

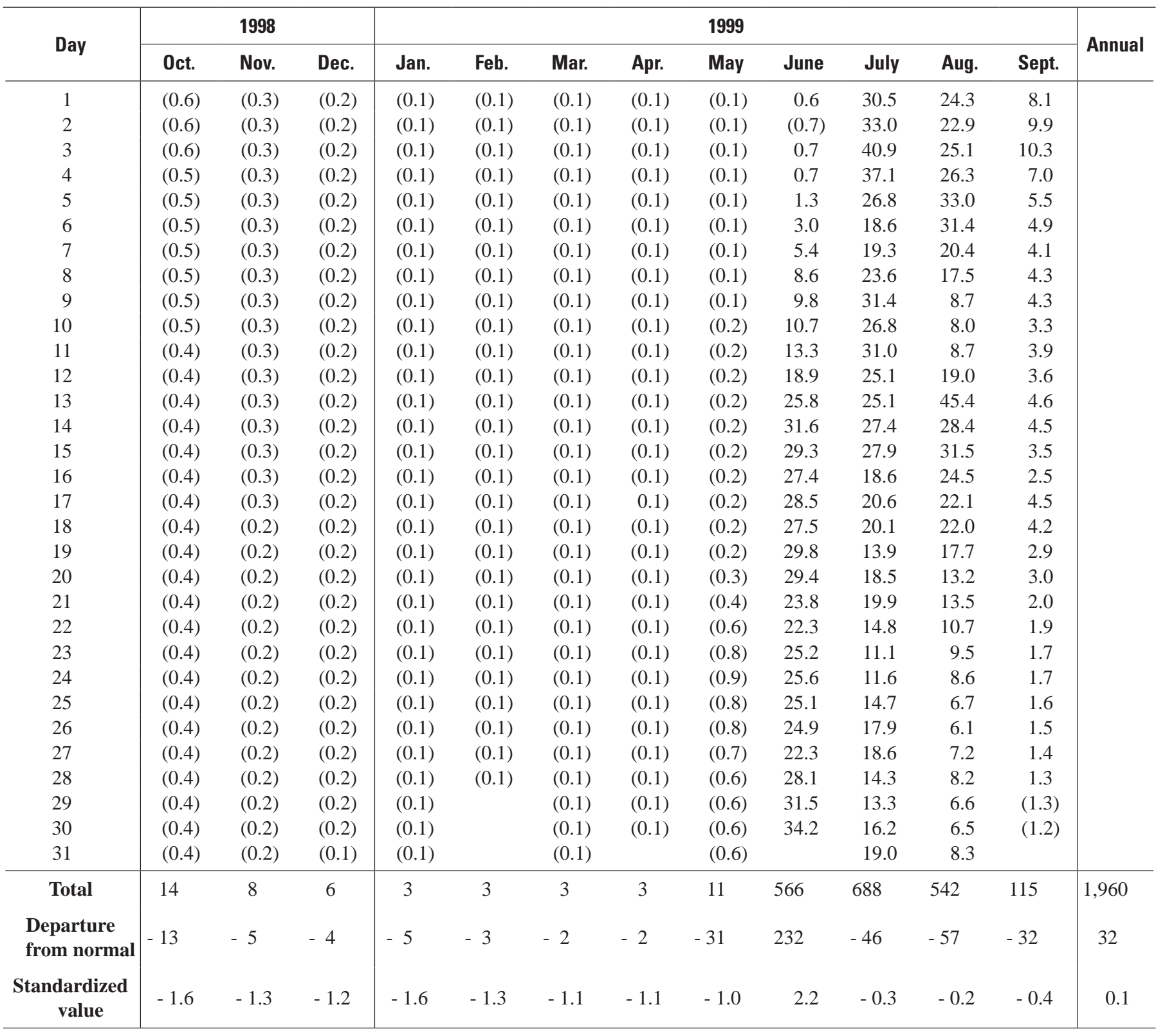


Table 35. Daily mean, monthly, and annual runoff from the Gulkana Glacier Basin, 2000 hydrologic year.

[Values in millimeters, averaged over the basin; except standardized values which are in units of standard deviation. Data in parentheses ( ) indicate value is estimated (see text for explanation)]

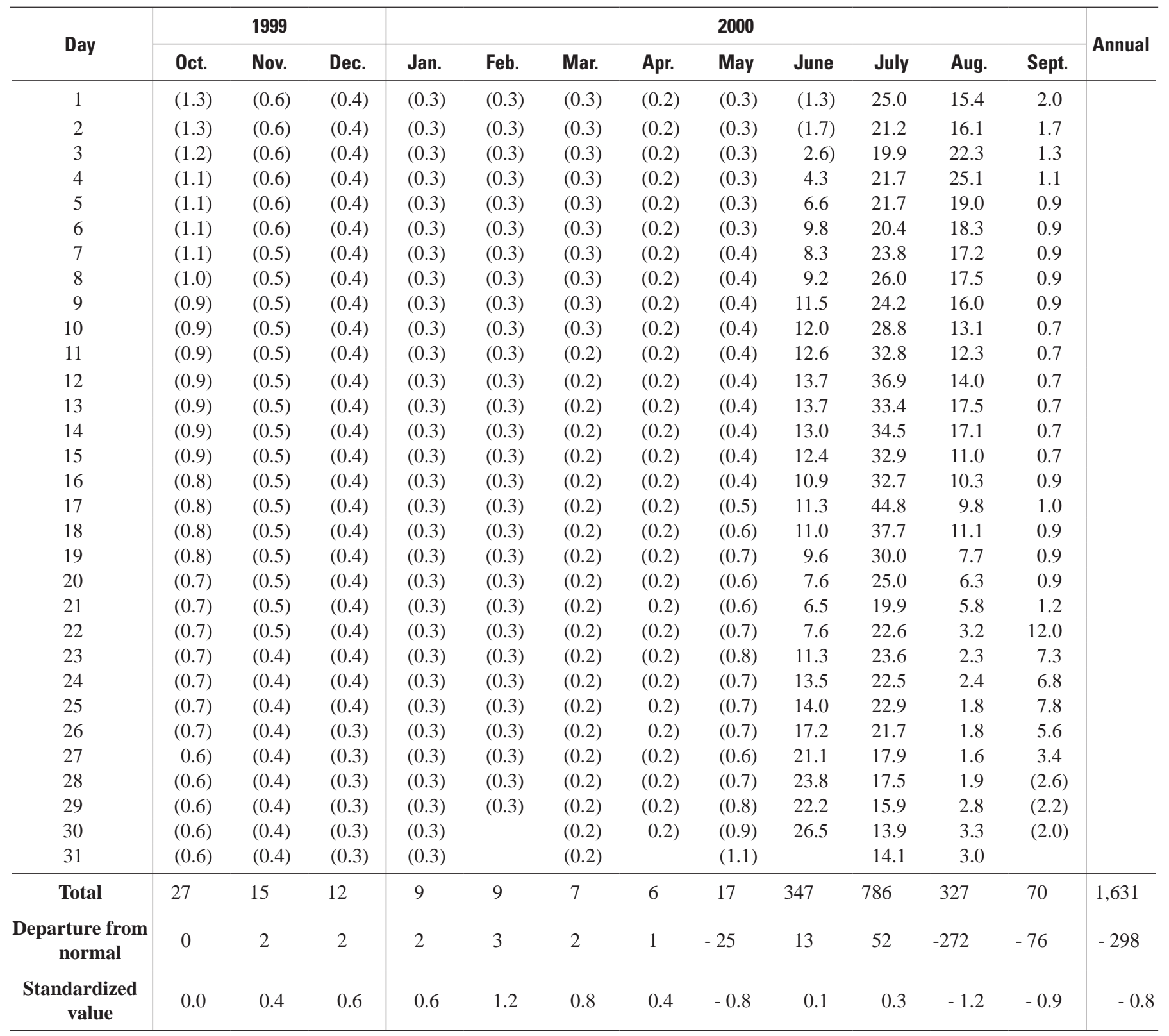


Table 36. Daily mean, monthly, and annual runoff from the Gulkana Glacier Basin, 2001 hydrologic year.

[Values in millimeters, averaged over the basin; except standardized values which are in units of standard deviation. Data in parentheses ( ) indicate value is estimated (see text for explanation)]

\begin{tabular}{|c|c|c|c|c|c|c|c|c|c|c|c|c|c|}
\hline Day & \multicolumn{3}{|c|}{2000} & \multicolumn{9}{|c|}{2001} & Annual \\
\hline 1 & 1.8 & 0.8 & 0.5 & 0.4 & 0.3 & 0.2 & 0.2 & 0.2 & 5.7 & 20.8 & 26.6 & 7.9 & \\
\hline 3 & 1.7 & 0.8 & 0.5 & 0.3 & 0.2 & 0.2 & 0.2 & 0.2 & 4.4 & 19.2 & 19.7 & 6.8 & \\
\hline 4 & 1.6 & 0.8 & 0.5 & 0.3 & 0.2 & 0.2 & 0.2 & 0.2 & 5.1 & 17.8 & 18.7 & 5.3 & \\
\hline 5 & 1.6 & 0.8 & 0.5 & 0.3 & 0.2 & 0.2 & 0.2 & 0.2 & 6.1 & 19.9 & 16.5 & 4.2 & \\
\hline 7 & 1.5 & 0.7 & 0.5 & 0.3 & 0.2 & 0.2 & 0.2 & 0.2 & 8.2 & 14.2 & 16.0 & 2.4 & \\
\hline 8 & 1.3 & 0.7 & 0.5 & 0.3 & 0.2 & 0.2 & 0.2 & 0.2 & 6.9 & 13.1 & 15.4 & 2.1 & \\
\hline 9 & 1.3 & 0.7 & 0.4 & 0.3 & 0.2 & 0.2 & 0.2 & 0.2 & 7.1 & 11.8 & 15.0 & 2.1 & \\
\hline 10 & 1.3 & 0.7 & 0.5 & 0.3 & 0.2 & 0.2 & 0.2 & 0.2 & 7.9 & 12.7 & 15.6 & 2.4 & \\
\hline 11 & 1.3 & 0.7 & 0.4 & 0.3 & 0.2 & 0.2 & 0.2 & 0.2 & 9.4 & 12.0 & 14.9 & 2.4 & \\
\hline 12 & 1.2 & 0.6 & 0.4 & 0.3 & 0.2 & 0.2 & 0.2 & 0.2 & 12.0 & 11.3 & 15.0 & 2.2 & \\
\hline 16 & 1.1 & 0.6 & 0.4 & 0.3 & 0.2 & 0.2 & 0.2 & 0.4 & 10.1 & 17.4 & 19.6 & 2.0 & \\
\hline 17 & 1.1 & 0.6 & 0.4 & 0.3 & 0.2 & 0.2 & 0.2 & 0.5 & 10.6 & 17.5 & 17.6 & 2.8 & \\
\hline 18 & 1.0 & 0.6 & 0.4 & 0.3 & 0.2 & 0.2 & 0.2 & 0.7 & 11.0 & 21.0 & 17.6 & 3.3 & \\
\hline 19 & 1.0 & 0.6 & 0.4 & 0.3 & 0.2 & 0.2 & 0.2 & 1.0 & 10.9 & 20.4 & 16.4 & 3.1 & \\
\hline 20 & $(1.0)$ & 0.6 & 0.4 & 0.3 & 0.2 & 0.2 & 0.2 & $(1.7)$ & 15.2 & 29.0 & 16.9 & 3.3 & \\
\hline 21 & $(1.0)$ & 0.6 & 0.4 & 0.3 & 0.2 & 0.2 & 0.2 & (2.2) & 19.2 & 37.5 & 22.2 & 3.0 & \\
\hline 22 & 0.9 & 0.5 & 0.4 & 0.3 & 0.2 & 0.2 & 0.2 & (1.8) & 17.5 & 48.5 & 21.2 & 2.1 & \\
\hline 23 & 0.9 & 0.5 & 0.4 & 0.3 & 0.2 & 0.2 & 0.2 & (1.3) & 19.9 & 47.0 & 15.9 & 2.0 & \\
\hline 24 & 0.9 & 0.5 & 0.4 & 0.3 & 0.2 & 0.2 & 0.2 & (1.1) & 19.8 & 45.1 & 15.6 & 1.8 & \\
\hline 25 & 0.9 & 0.5 & 0.4 & 0.3 & 0.2 & 0.2 & 0.2 & 1.0 & 17.7 & 39.7 & 14.6 & 1.7 & \\
\hline 26 & 0.9 & 0.5 & 0.4 & 0.3 & 0.2 & 0.2 & 0.2 & 1.1 & 25.1 & 37.5 & 12.5 & 1.6 & \\
\hline 27 & $(0.9)$ & 0.5 & 0.4 & 0.3 & 0.2 & 0.2 & 0.2 & 1.3 & 23.1 & 33.4 & 11.6 & 1.5 & \\
\hline $\begin{array}{c}\text { Standardized } \\
\text { value }\end{array}$ & 1.2 & 1.4 & 1.0 & 0.6 & -0.0 & 0.3 & 0.4 & -0.5 & 0.4 & 0.3 & -0.4 & -0.7 & -0.1 \\
\hline
\end{tabular}

Mean annual runoff for the period of record is about $1.9 \mathrm{~m}$ (fig. 16). Monthly runoff normally peaks in July $(0.7 \mathrm{~m})$ with August a close second $(0.6 \mathrm{~m})$, and June a distant third $(0.3 \mathrm{~m})$. The cumulative sum of estimated winter runoff represents about 15 percent of the annual runoff. Annual runoff was well above normal in 1997 and 1998, about normal in 1999 and 2001, and well below normal in 2000. The average runoff for the 5-year period (1997-2001) shown in figure 16 is 9 percent greater than the average prior to 1997. We estimate a 12 percent augmentation in runoff attributable to glacier shrinkage when considering all available data. Recent data (1997-2001) demonstrate a larger, 22 percent augmentation of runoff. The augmentation is the ratio of the glacier averaged mass balance (converted to a basin-averaged value) to the basin averaged runoff for similar time periods and expressed as a percent. 


\section{Summary}

The 1997-2001 measured winter snow, maximum winter snow, summer, net, and annual mass balances in the Gulkana Glacier basin were evaluated on the basis of meteorological, hydrological, and glaciological data. Conventional and reference surface mass balances are reported.

Seasonal values for the mass balance, surface altitude, and ice motion at index measurement sites and the glacier wide mass balance are reported. Winter balance for the 5-year period discussed in this report was about equal to the previous 31-year average (1.1 mweq) and exhibits no overall trend. The 1997-2001 average net balance of -0.77 mweq was about 2.5 times more negative than the previous 31-year average of -0.30 mweq. The trend indicates a decreasing net balance by -0.2 mweq/decade. The 1997 net balance, -1.71 mweq, was the most negative year of record. The 1997-2001 average summer balance of -1.92 mweq was almost 1 standard deviation below the previous 31-year average of -1.41 mweq. The trend indicates a decreasing summer balance by -0.18 mweq/decade. The 1997 summer balance, -2.76 mweq, was the most negative year of record.

Daily values of air temperature, precipitation catch, and wind speed from the Gulkana Glacier weather station are reported. Evaluation of a permanent air temperature sensor change is reported. Mean annual air temperature at an altitude of $1,480 \mathrm{~m}$ was about $-3.1^{\circ} \mathrm{C}$ for the $1968-2001$ and indicates a warming trend of $+0.16^{\circ} \mathrm{C} /$ decade, mostly due to increased winter temperatures. This translates into mean annual temperature at the 1966-2001 average ELA of $5.0^{\circ} \mathrm{C}$. Precipitation catch averages $1.0 \mathrm{~m}$ for 1968-2001, with a drying trend of 2-3 percent per decade in the annual and summer values, but no trend in the winter values. Catch efficiency of the gage is only 60 percent on average, so true precipitation is about 60 percent greater than the catch, or on average $1.6 \mathrm{~m}$. Gage testing at a facility in Canada showed that the catch efficiency of our wind-shielded gage for solid precipitation is reduced from 100 percent by about 10 percent for every $1 \mathrm{~m} / \mathrm{s}$ increase in wind speed. Winds average $3.5 \mathrm{~m} / \mathrm{s}$ but are greater than $5-8 \mathrm{~m} / \mathrm{s}$, the threshold for blowing snow, 15-20 percent of the time.

Annual values for the area altitude distribution, equilibrium line altitude, and accumulation area ratio are reported. Additionally, an AAD was reported for 1900 and annually from 1966 to 2001. Annual terminus/lower-glacier position is included plus that for 1985 and 1993. AADs show a decline in area since the peak of the Little Ice Age in 1900. The rate of decline has been faster since 1993. As of 2001, the glacier has lost about $8.2 \mathrm{~km}^{2}$ or 33 percent of its area since 1900 , and about $2.8 \mathrm{~km}^{2}$ or 14 percent of its area since 1966. The 1997-2001 average AAR is 0.53 compared to the previous 31-year average of 0.60 . The 1997-2001 average ELA is $35 \mathrm{~m}$ higher than the previous 31-year average of 1,760 m. The 1997-2001 glacier surface altitude data demonstrates continued glacier-wide thinning trend of the 1990s, except for site D where recent thickening has been measured.

Ice motion for $1997-2001$ was $10-15 \mathrm{~m} / \mathrm{yr}$ at site $\mathrm{A}$ in the ablation zone, $30-40 \mathrm{~m} / \mathrm{yr}$ at site B in the central reach of the glacier, and 35-50 m/yr at site D in the lower accumulation zone. A general slowing trend occurred through the 1990s with a reduction in speed of about 40 percent since 1990. Motion varies seasonally with summer speeds 20-30 percent greater than winter speeds.

From 1949 to 1996, the terminus retreated about 1,650 m yielding an average retreat rate of $35 \mathrm{~m} / \mathrm{yr}$. Since then it has been retreating 15-20 m/yr along the south margin where the terminus is best defined and fed by the main trunk of the glacier and two to three times faster along the southwest margin. Faster retreat is expected to continue along the southwest margin as source ice for this area is cutoff by thinning ice coming over the icefall just upstream.

Runoff for 1997-2001 was 7 percent greater than the previous period of record (1967-78, 1990-96) average, about $1.9 \mathrm{~m}$. Runoff has been augmented by the shrinkage of the glacier in 19 of the total 24 years of runoff measurements. For the period of record prior to 1997, glacier shrinkage made up an average of 13 percent of the runoff. For the 5-year period of this study, 1997-2001, glacier shrinkage contributed an average of 25 percent of the runoff.

\section{References Cited}

Barry, R.G., 1989, Climate of the Arctic Ocean, in Herman, Y., ed., The Arctic Seas-climatology, oceanography, geology, and biology: New York, Van Nostrand Reinhold, p. 1-46.

Bidlake, W.R., Josberger, E.G., and Savoca, M.E., 2007, Water, ice, and meteorological measurements at south Cascade Glacier, Washington, balance years 2004 and 2005: U.S. Geological Survey Scientific Investigations Report 2007-5055, 70 p., accessed June 11, 2009, at http://pubs. water.usgs.gov/sir20075055/.

Bitz, C.M., and Battisti, D.S., 1999, Interannual to decadal variability in climate and the glacier mass balance in Washington, Western Canada, and Alaska: Journal of Climate, v. 12, p. 3181-3196, accessed July 12, 2010, at http://www.atmos.washington.edu/ david/bb99.pdf.

Braithwaite, R.J., 1995, Positive degree-day factors for ablation on the Greenland ice sheet studied by energybalance modelling: Journal of Glaciology, v. 41, no. 137, p. 153-160. 
Braithwaite, R.J., and Olesen, O.B., 1985, Ice ablation in West Greenland in relation to air temperature and global radiation: Zeitschrift für Gletscherkunde und Glazialgeologie, v. 20, no. 1984, p. 155-168.

Braithwaite, R.J., and Olesen, O.B., 1993, Seasonal variation of ice ablation at the margin of the Greenland ice sheet and its sensitivity to climate change, Qamanârssûp sermia, West Greenland: Journal of Glaciology, v. 39, no. 132, p. 267274.

Cheney, Ward, and Kincaid, David, 1980, Numerical mathematics and computing (2d ed.): Monterey, Calif., Brooks/Cole Publishing Co., 562 p.

Cox, L.H., 2002, Comparison of geodetic and glaciological mass balance on Gulkana Glacier, Alaska: Fairbanks, University of Alaska, M.S. thesis, 44 p., accessed June 16, 2009, at http://ak.water.usgs.gov/glaciology/gulkana/ reports/2002 Leif Cox MS Thesis.pdf.

Cox, L.H., and March, R.S., 2004, Comparison of geodetic and glaciological mass-balance techniques, Gulkana Glacier, Alaska, U.S.A.: Journal of Glaciology, v. 50, no. 170, p. 363-370, accessed April 28, 2008, at http://ak.water. usgs.gov/glaciology/gulkana/reports/2004 geodetic vs glaciologic/index.htm.

Dowdeswell, J.A., Hagen, J.O., Björnsson, Helgi, Glazovsky, A.F., Harrison, W.D., Holmlund, Per, Jania, Jacek, Korner, R.M., Lefauconnier, Bernard, Ommanney, C.S.L., and Thomas, R.H., 1997, The mass balance of circum-arctic glaciers and recent climate change: Quaternary Research, v. 48 , issue 1 , p. $1-14$.

Elsberg, D.H., Harrison, W.D., Echelmeyer, K.A., and Krimmel, R.M., 2001, Quantifying the effects of climate and surface change on glacier mass balance: Journal of Glaciology, v. 47, no. 159, p. 649-658.

Fahl, C.B., 1973, Some relationships between glaciers and climate in Alaska: Fairbanks, University of Alaska, Ph.D. dissertation, $191 \mathrm{p}$.

Fountain, A.G., Krimmel, R.M., and Trabant, D.C., 1997, A strategy for monitoring glaciers: U.S. Geological Survey Circular 1132, 19 p., accessed July 1, 2003, at http://pubs. water.usgs.gov/cir1132.

Goodison, B.E., Louie, P.Y.T., and Yang, Daqing, 1998, WMO solid precipitation measurement intercomparison, final report: World Meteorological Organization, WMO Instrument and Observing Methods no. 67, 212 p.

Haeberli, Wilfried, 1985, Fluctuations of glaciers 1975-1980 (v. IV): International Association of Hydrologic Sciences (International Commission of Snow and Ice) and United Nations Educational, Scientific and Cultural Organization, $265 \mathrm{p}$.
Haeberli, Wilfried, and Herren, Eveline, eds., 1991, Glacier mass balance bulletin no. 1: ETH Zurich, World Glacier Monitoring Service, International Association of Hydrologic Sciences (International Commission of Snow and Ice) and United Nations Educational, Scientific and Cultural Organization, $70 \mathrm{p}$.

Haeberli, Wilfried, Herren, Eveline, and Hoelzle, Martin, eds., 1993, Glacier mass balance bulletin no. 2: ETH Zurich, World Glacier Monitoring Service, International Association of Hydrologic Sciences (International Commission of Snow and Ice) and United Nations Educational, Scientific and Cultural Organization, 74 p.

Haeberli, Wilfried, and Hoelzle, Martin, 1993, Fluctuations of glaciers 1985-1990 (v. VI): International Association of Hydrologic Sciences (International Commission on Snow and Ice) and United Nations Environment Programme and United Nations Educational, Scientific and Cultural Organization, 322 p.

Haeberli, Wilfried, Hoelzle, Martin, and Bösch, H., eds., 1994, Glacier mass balance bulletin no. 3: ETH Zurich, World Glacier Monitoring Service, International Association of Hydrologic Sciences (International Commission of Snow and Ice) and United Nations Educational, Scientific and Cultural Organization, $80 \mathrm{p}$.

Haeberli, Wilfried, Hoelzle, Martin, and Frauenfelder, Regula, eds., 1999, Glacier mass balance bulletin no. 5: University and ETH Zurich, World Glacier Monitoring Service, International Association of Hydrologic Sciences (International Commission of Snow and Ice) and United Nations Educational, Scientific and Cultural Organization, 96 p., accessed July 1, 2003.

Haeberli, Wilfried, Hoelzle, Martin, and Suter, Stephen, eds., 1996, Glacier mass balance bulletin no. 4: University and ETH Zurich, World Glacier Monitoring Service, International Association of Hydrologic Sciences (International Commission of Snow and Ice) and United Nations Educational, Scientific and Cultural Organization, 90 p., accessed July 1, 2003, at http://www.geo.unizh.ch/ wgms/mbb/mbb4/mbb4.html.

Haeberli, Wilfried, Hoelzle, Martin, Suter, Stephen, and Frauenfelder, Regula, 1998, Fluctuations of glaciers 1990-1995 (v. VII): International Association of Hydrologic Sciences (International Commission on Snow and Ice) and United Nations Environment Programme and United Nations Educational, Scientific and Cultural Organization, 296 p., accessed July 1, 2003. 
Haeberli, Wilfried, and Müller, Peter, 1988, Fluctuations of glaciers 1980-1985 (v. V): International Association of Hydrologic Sciences (International Commission on Snow and Ice) and United Nations Environment Programme and United Nations Educational, Scientific and Cultural Organization, $290 \mathrm{p}$.

Harrison, W.D., Cox, L.H., Hock, R., March, R.S., and Pettit, E.C., 2009, Implications for the dynamic health of a glacier from comparison of conventional and referencesurface balances: Annals of Glaciology, v. 50, p. 25-30, accessed July 12, 2010, at http://digitalcommons.unl.edu/ usgsstaffpub/88/.

Harrison, W.D., Elsberg, D.H., Cox, L.H., and March, R.S., 2005, Different balances for climatic and hydrologic applications. Journal of Glaciology, v. 51, no. 172, p. 176., accessed July 12, 2010, at http://ak.water.usgs. gov/glaciology/gulkana/reports/2005.01\%20J\%20of\%20 Gl\%20Harrison\%20Elsberg\%20Cox\%20March\%20 Correspondence.pdf.

Hodge, S.M., Trabant, D.C., Krimmel, R.M., Heinrichs, T.A., March, R.S., and Josberger, E.G., 1998, Climate variations and changes in mass of three glaciers in western North America: Journal of Climate, v. 11, no. 9, p. 2161-2179.

Jóhannesson, Tómas, Sigurdsson, Oddur, Laumann, Tron, and Kennett, Michael, 1995, Degree-day glacier mass-balance modelling with applications to glaciers in Iceland, Norway, and Greenland: Journal of Glaciology, v. 41, no. 138, p. 345-358.

Josberger, E.G., Bidlake, W.R., March, R.S., and Kennedy, B.W., 2006, Glacier mass-balance fluctuations in the Pacific Northwest and Alaska, USA [poster]: International Symposium on Cryospheric Indicators of Global Climate Change, August 21-25, 2006, Cambridge, United Kingdom, accessed April 28, 2008, at http://ak.water.usgs.gov/ glaciology/reports/2006 igs/index.htm.

Josberger, E.G., Bidlake, W.R., March, R.S., and Kennedy, B.W., 2007, Glacier mass-balance fluctuations in the Pacific Northwest and Alaska, USA: Annals of Glaciology, v. 46, p. 291-296, accessed April 28, 2008, at http://ak.water.usgs. gov/glaciology/reports/2007 3glacier\%20balance/index. htm.

Karl, T.R., Tarpley, J.D., Quayle, R.G., Diaz, H.F., Robinson, D.A., and Bradley, R.S., 1989, The recent climate recordWhat it can and cannot tell us: Reviews of Geophysics, v. 27, no. 3, p. 405-430, accessed July 12, 2010, at http://www. geo.umass.edu/faculty/bradley/karl1989.pdf.

Kasser, Peter, 1967, Fluctuations of glaciers 1959-1965 (v. I): International Association of Scientific Hydrology (International Commission of Snow and Ice) and United Nations Educational, Scientific and Cultural Organization (no pagination).
Kennedy, B.W., 1995, Air temperature and precipitation data, Wolverine Glacier basin, Alaska, 1967-94: U.S. Geological Survey Open-File Report 95-444, 79 p., plus diskette.

Kennedy, B.W., Mayo, L.R., Trabant, D.C., and March, R.S., 1997, Air temperature and precipitation data, Gulkana Glacier, Alaska, 1968-96: U.S. Geological Survey OpenFile Report 97-358, 144 p., accessed July 3, 2003, at http:// ak.water.usgs.gov/glaciology/gulkana/reports/ofr97-358/.

Letréguilly, Anne, and Reynaud, Louis, 1989, Spatial patterns of mass-balance fluctuations of North American glaciers: Journal of Glaciology, v. 35, no. 120, p. 163-168.

Linn, K.R., Shaw, S.K., Swanner, W.C., Rickman, R.L., and Schellekens, M.F., 1997, Water resources data for Alaska, water year 1996: U.S. Geological Survey Water-Data Report AK-96-1, 270 p.

March, R.S., 1998, Mass balance, meteorological, ice motion, surface altitude, and runoff data at Gulkana Glacier, Alaska, 1994 balance year: U.S. Geological Survey WaterResources Investigations Report 97-4251, 31 p., accessed July 3, 2003, at http://pubs.water.usgs.gov/wri97-4251.

March, R.S., 2000, Mass balance, meteorological, ice motion, surface altitude, runoff, and ice thickness data at Gulkana Glacier, Alaska, 1995 balance year: U.S. Geological Survey Water-Resources Investigations Report 00-4074, 33 p., accessed July 3, 2003, at http://pubs.water.usgs.gov/wri004074/.

March, R.S., 2003, Mass balance, meteorology, area altitude distribution, glacier-surface altitude, ice motion, terminus position, and runoff at Gulkana Glacier, Alaska, 1996 balance year: U.S. Geological Survey Water-Resources Investigations Report 03-4095, 33 p., accessed May 1, 2008, at http://ak.water.usgs.gov/glaciology/gulkana/ reports/wrir03-4095/index.htm.

March, R.S., and Trabant, D.C., 1996, Mass balance, meteorological, ice motion, surface altitude, and runoff data at Gulkana Glacier, Alaska, 1992 balance year: U.S. Geological Survey Water-Resources Investigations Report 95-4277, 32 p., accessed July 3, 2003, at http://pubs.water. usgs.gov/wri95-4277.

March, R.S., and Trabant, D.C., 1997, Mass balance, meteorological, ice motion, surface altitude, and runoff data at Gulkana Glacier, Alaska, 1993 balance year: U.S. Geological Survey Water-Resources Investigations Report 96-4299, 30 p., accessed July 3, 2003, at http://ak.water. usgs.gov/glaciology/gulkana/reports/wrir96-4299/.

Mayo, L.R., 1992, Internal ablation—an overlooked component of glacier mass balance [abs.]: Eos, Transactions, American Geophysical Union, v. 73, no. 43, supplement, p. 180. 
Mayo, L.R., March, R.S., and Trabant, D.C., 1992, Air temperature and precipitation data, 1967-88, Wolverine Glacier basin, Alaska: U.S. Geological Survey Open-File Report 91-246, 80 p.

Mayo, L.R., Meier, M.F., and Tangborn, W.V., 1972, A system to combine stratigraphic and annual mass-balance systems: A contribution to the International Hydrologic Decade: Journal of Glaciology, v. 11, no. 61, p. 3-14., accessed April 28, 2008, at http://ak.water.usgs.gov/glaciology/ reports/1972 mayo meier tangborn/index.htm.

Mayo, L.R., and Trabant D.C., 1982, Geodetic trisection, altitude, and ice-radar surveying techniques used at Knik Glacier, Alaska, and summary of 1979, 1980, and 1981 data: U.S. Geological Survey Open-File Report 82-685, 26 p.

Mayo, L.R., and Trabant, D.C., 1986, Recent growth of Gulkana Glacier, Alaska Range, and its relation to glacierfed runoff, in Subitzky, Seymour, ed., Selected papers in the hydrologic sciences: U.S. Geological Survey Water-Supply Paper 2290, p. 91-99., accessed June 15, 2009, at http:// ak.water.usgs.gov/glaciology/gulkana/reports/1986 recent growth of gulkana/1986 Recent growth of Gulkana - Mayo \& Trabant - WSP2290.pdf.

Mayo, L.R., Trabant, D.C., and March, R.S., 2004, A 30-year record of surface mass balance (1966-95), and motion and surface altitude (1975-95) at Wolverine Glacier, Alaska: U.S. Geological Survey Open-File Report 20041069, 105 p. (Also available at http://pubs.water.usgs.gov/ ofr20041069.)

Mayo, L.R., Trabant, D.C., March, R.S., and Haeberli, Wilfried, 1979, Columbia Glacier stake locations, mass balance, glacier surface altitude, and ice radar data, 1978 measurement year: U.S. Geological Survey Open-File Report 79-1168, 79 p.

Meier, M.F., 1965, Glaciers and climate, in Wright, H.E., Jr., and Frey, D.G., eds., The Quaternary of the United States: Princeton, N.J., Princeton University Press, p. 795-805.

Meier, M.F., Mayo, L.R., Trabant, D.C., and Krimmel, R.M., 1980, Comparison of mass balance and runoff at four glaciers in the United States, 1966 to 1977: Academy of Sciences of USSR, Section of Glaciology, Data of Glaciological Studies, Publication No. 38, p. 138-147 (Russian text with figures), p. 214-219 (English text).

Meier, M.F., Tangborn, W.V., Mayo, L.R., and Post, Austin, 1971, Combined ice and water balances of Gulkana and Wolverine Glacier, Alaska, and South Cascade Glacier, Washington, 1965 and 1966 hydrologic years: U.S. Geological Survey Professional Paper 715-A, 23 p., accessed April 28, 2008, at http://ak.water.usgs.gov/ glaciology/reports/1971_pp715A/index.htm.
Muller, Fritz, 1977, Fluctuations of glaciers 1970-1975 (v. III): International Association of Hydrologic Sciences (International Commission of Snow and Ice) and United Nations Educational, Scientific and Cultural Organization, 269 p.

National Climatic Data Center, 1996, Climatological data annual summary, Alaska, 1995: v. 81, no. 13, 23 p.

Østrem, Gunnar, and Brugman, M., 1991, Glacier massbalance measurements: A manual for field and office work: Norwegian Water Resources and Energy Administration, and Environment Canada, National Hydrology Research Institute Science Report no. 4, 224 p.

Paterson, W.S.B., 1994, The Physics of Glaciers. Third edition. Oxford, etc., Elsevier.

Péwé, T.L., and Reger, R.D., 1983, Delta River area, Alaska Range, in Péwé, T.L., and Reger, R.D., eds., Guidebook to permafrost and Quaternary geology along the Richardson and Glenn Highways between Fairbanks and Anchorage, Alaska: Fourth International Conference on Permafrost: Alaska Division of Geological and Geophysical SurveysGuidebook 1, p. 47-135., accessed April 28, 2008, at http:// www.dggs.alaska.gov/webpubs/dggs/gb/text/gb001.PDF.

Rasmussen, L.A., and Conway, H., 2004, Climate and glacier variability in western North America. Journal of Climate. v. 17, no. 9, p. 1804-1815., accessed April 28, 2008, at http:// earthweb.ess.washington.edu/ lar/p48text.pdf.

Tangborn, W.V., Mayo, L.R., Scully, D.R., and Krimmel, R.M., 1977, Combined ice and water balances of Maclure Glacier, California, South Cascade Glacier, Washington, and Wolverine and Gulkana Glaciers, Alaska, 1967 hydrologic year: U.S. Geological Survey Professional Paper 715-B, 20 p., accessed April 20, 2008, at http://ak.water.usgs.gov/ glaciology/reports/1977 pp715B/index.htm.

Trabant, D.C., and March, R.S., 1999, Mass-balance measurements in Alaska and suggestions for simplified observation programs: Geografiska Annaler, Series A, Physical Geography, v. 81, no. 4, p. 777-789.

Trabant, D.C., March, R.S., and Kennedy, B.W., 1998, Glacier mass-balance trends in Alaska and climate-regime shifts [abs.]: Eos, Transactions, American Geophysical Union, v. 79, no. 48, p. F277, accessed July 3, 2003, at http://ak.water. usgs.gov/glaciology/reports/AGU98/98AGU balance trend poster.htm.

Trabant, D.C., and Mayo, L.R., 1985, Estimation and effects of internal accumulation on five glaciers in Alaska: Annals of Glaciology, v. 6, p. 113-117. 
U.S. Departments of the Army and the Air Force, 1951, TM 5-241- to 16-1-233, The universal grid systems (Universal Transverse Mercator) and (Universal Polar Stereographic): Washington, Government Printing Office, 324 p.

U.S. Geological Survey, 1968-2002, Water resources data for Alaska, water years 1967-2001: U.S. Geological Survey Water-Data Report 67-1 to 70-1 and AK-71-1 to AK-01-1 (published annually; AK designation not used before 1971; since 2000). (Also available at http://wdr.water.usgs.gov/.)

UNESCO, 1970, Combined heat, ice and water balances at selected glacier basins: United Nations Educational, Scientific, and cultural organization, International Association of Scientific Hydrology, Technical papers in hydrology, no. 5, 20 p.
Wahl, K.L., Thomas, W.O., Jr., and Hirsch, R.M., 1995, The stream-gaging program of the U.S. Geological Survey: U.S. Geological Survey Circular 1123, 22 p., accessed June 26, 2009, at http://pubs.er.usgs.gov/usgspubs/cir/cir1123.

Walters, R.A., and Meier, M.F., 1989, Variability of glacier mass balances in western North America, in Peterson, D.H., Aspects of climate variability in the Pacific and Western Americas: American Geophysical Union, Geophysical Monograph 55, p. 365-374.

Wilson, B.D., Yinger, C.H., Feess, W.A., and Shank, C.C., 1999, New and improved: The broadcast interfrequency biases: GPS World, v. 10, no. 9, p. 56-64. 
This page intentionally left blank. 


\section{Appendix A. Coordinate Conversions}

\section{Horizontal Coordinates}

Horizontal coordinates can be converted between the local sea-level-scale system and UTM, NAD 83 datum coordinates by:

$$
\text { UTM Easting }=\bar{k} X_{L}+575,000 \mathrm{~m},
$$

and

$$
\text { UTM Northing }=\bar{k} Y_{L}+7,011,000 \mathrm{~m} \text {, }
$$

where $\bar{k}$ is the mean horizontal scale factor between the UTM plane and sea level and $X_{L}$ and $Y_{L}$ are local coordinates, in meters.

The scale factor, $k$, at a point is a variable defined by:

$$
k=\frac{0.9996}{\sin \left(100+\frac{500,000-E}{100,000}\right)},
$$

where $E$ is the UTM Easting of the point and the trigonometric function is evaluated in grad.

The mean scale factor used in equations 1 and 2 is the mean value of the nonlinear distribution of scale factors between the local origin and an observation point. $E$ is approximated by the sum of the UTM Easting of the local origin $(575,000 \mathrm{~m})$ and $X_{L}$.

$$
\bar{k}=\frac{1}{X_{L}} \int_{(575,000)}^{\left(575,000+X_{L}\right.} k d E .
$$

Equation 5 is accurate within about 0.1 part per million of equations from U.S. Departments of the Army and the Air Force (1951, specifically see page 219 and appendixes IV and V) and, hence, yields results accurate at the centimeter level.

The mean scale factor is estimated using Simpson's rule (Cheney and Kincaid, 1980):

$$
\begin{aligned}
\bar{k} & =\frac{0.9996}{6} \frac{1}{\sin \left(100+\frac{500,000-575,000}{100,000}\right)} \\
& +\frac{1}{\sin \left(100+\frac{500,000-575,000-1 / 2 X_{L}}{100,000}\right)} \\
& +\frac{1}{\sin \left(100+\frac{5000,000-575,000-X_{L}}{100,000}\right)} .
\end{aligned}
$$

\section{Vertical Coordinates}

Base on the five nearest National Geodetic Survey (NGS) vertical benchmarks with stability rating of $\mathrm{A}$ or $\mathrm{B}$, the local conversion from NGVD 29 to NAVD 88 is a constant equal to $1.64 \pm 0.01 \mathrm{~m}$ (the later datum being higher).

A series of Geoid models have been developed by NGS to convert between NAD 83 GPS derived ellipsoid heights and NAVD 88 orthometric heights. For Alaska these models include GEOID99 and GEOID06 (accessed June 22, 2009, at http://www.ngs.noaa.gov/TOOLS/program_descriptions.html). GEOID06 is an update of GEOID99 that covers only Alaska. These models have significant errors at Gulkana Glacier. From optical and GPS surveys, the Geoid height is generally 16-17 meters in Gulkana area. Both GEOID models generate geoid heights that typically are $1.5 \mathrm{~m}$ too small in the vicinity of Gulkana.

A local geoid model for Gulkana Glacier basin derived from a planar best fit between the seven best quality optical and GPS surveys of off-glacier survey monuments is:

$$
\begin{aligned}
\text { geoid height, in meters } & =172.0983 \\
& +0.0000372429 * \text { UTM Easting } \\
& +0.0000238585 * \text { UTM Northing, (A6) }
\end{aligned}
$$

where UTM coordinates are zone 6, NAD 83 datum, and:

$$
\begin{aligned}
\text { NGVD } 29 \text { height } & =\text { NAD 83ellipsoid height } \\
& - \text { geoid height }
\end{aligned}
$$

The standard error of height estimates from this conversion is $\pm 0.06 \mathrm{~m}$ based on measurements at 11 sites. The slope of this locally defined geoid model is similar the slope of the GEOID06. 
72 Gulkana Glacier, Alaska—Mass Balance, Meteorology, and Water Measurements, 1997-2001

This page intentionally left blank. 
Publishing support provided by the U.S. Geological Survey Publishing Network, Tacoma Publishing Service Center

For more information concerning the research in this report, contact the Director, Alaska Science Center

U.S. Geological Survey

4210 University Dr.

Anchorage, Alaska 99508-4650

http://alaska.usgs.gov 
ฏ 